\begin{tabular}{|l|l|l|l|l|l|l|l|l|l|l|l|l|l|l|l|l|l|l|l|}
\hline RID & G & AG & D & Edu & Eth & Job & SES & FZ & HF & DHF & Inc1 & Inc2 & Q1 & Q2 & Q3 & Q4 & O1 & O2 & O3 \\
\hline
\end{tabular} \begin{tabular}{|l|l|r|r|r|r|r|r|r|r|r|r|r|r|r|r|r|r|r|r|}
10111 & 1 & 4 & 2 & 3 & 3 & 2 & 3 & 2 & 1 & 2 & 1 & 1 & 1 & 1 & 1 & 1 & 0 & 0 & 0 \\
10112 & 1 & 2 & 2 & 5 & 3 & 5 & 3 & 1 & 1 & 2 & 0 & 0 & 1 & 1 & 1 & 1 & 0 & 0 & 0 \\
\hline
\end{tabular}

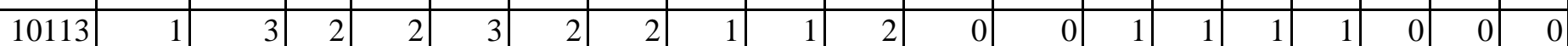

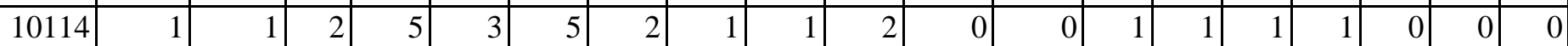
\begin{tabular}{|l|l|l|l|l|l|l|l|l|l|l|l|l|l|l|l|l|r|r|r|}
\hline 10115 & 1 & 4 & 2 & 3 & 3 & 5 & 2 & 1 & 1 & 2 & 1 & 1 & 1 & 1 & 1 & 1 & 0 & 0 & 0 \\
\hline
\end{tabular}

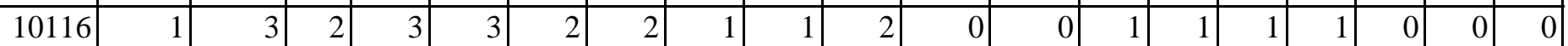

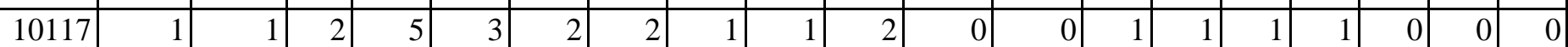
\begin{tabular}{|l|l|l|l|l|l|l|l|l|l|l|l|l|l|l|l|l|r|r|r|}
10118 & 1 & 3 & 2 & 4 & 3 & 5 & 2 & 1 & 1 & 2 & 1 & 0 & 1 & 1 & 1 & 1 & 0 & 0 & 0 \\
\hline
\end{tabular}

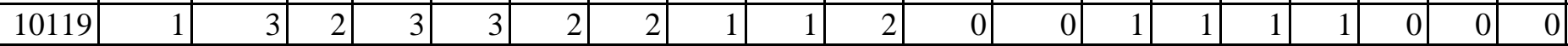
\begin{tabular}{|l|l|l|l|l|l|l|l|l|l|l|l|l|l|l|l|l|l|l|l|}
101110 & 1 & 4 & 2 & 2 & 3 & 2 & 2 & 1 & 1 & 2 & 0 & 0 & 1 & 1 & 1 & 1 & 0 & 0 & 0 \\
\hline
\end{tabular} \begin{tabular}{|l|l|l|l|l|l|l|l|l|l|l|l|l|l|l|l|l|l|l|l|}
101111 & 1 & 3 & 2 & 2 & 3 & 2 & 2 & 2 & 1 & 2 & 0 & 0 & 1 & 1 & 1 & 1 & 0 & 0 & 0 \\
\hline
\end{tabular}

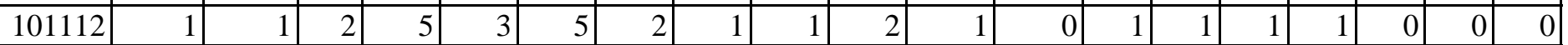
\begin{tabular}{|l|l|l|l|l|l|l|l|l|l|l|l|l|l|l|l|l|r|r|r|}
\hline 101113 & 1 & 4 & 2 & 4 & 3 & 5 & 2 & 1 & 2 & 2 & 1 & 1 & 1 & 1 & 1 & 1 & 0 & 0 & 0 \\
\hline
\end{tabular} \begin{tabular}{|l|l|l|r|r|r|r|r|r|r|r|r|r|r|r|r|r|r|r|r|}
\hline 101114 & 1 & 5 & 2 & 5 & 3 & 5 & 3 & 1 & 1 & 2 & 1 & 1 & 1 & 1 & 1 & 1 & 0 & 0 & 0 \\
\hline
\end{tabular} \begin{tabular}{|r|r|r|r|r|r|r|r|r|r|r|r|r|r|r|r|r|r|r|r|}
\hline 101115 & 0 & 5 & 2 & 1 & 3 & 2 & 1 & 1 & 1 & 2 & 0 & 0 & 0 & 99 & 99 & 99 & 99 & 99 & 99 \\
\hline 10116 & 0 & 2 & 2 & 3 & 3 & 1 & 2 & 2 & 1 & & 0 & 0 & 1 & 1 & & 1 & 0 & 0 & 0 \\
\hline
\end{tabular} \begin{tabular}{|l|l|l|l|l|l|l|l|l|l|l|l|l|l|l|r|r|r|r|r|}
\hline 101116 & 0 & 2 & 2 & 3 & 3 & 1 & 2 & 2 & 1 & 1 & 0 & 0 & 1 & 1 & 1 & 1 & 0 & 0 & 0 \\
\hline
\end{tabular} \begin{tabular}{|l|l|l|l|l|l|l|l|l|l|l|l|l|l|l|r|r|r|r|r|}
\hline 101117 & 1 & 3 & 2 & 5 & 3 & 5 & 3 & 1 & 1 & 1 & 1 & 1 & 1 & 1 & 2 & 1 & 1 & 0 & 1 \\
\hline
\end{tabular} \begin{tabular}{|l|l|r|r|r|r|r|r|r|r|r|r|r|r|r|r|r|r|r|r|}
\hline 101118 & 1 & 4 & 2 & 3 & 3 & 2 & 2 & 1 & 1 & 1 & 0 & 0 & 1 & 1 & 1 & 1 & 0 & 0 & 0 \\
\hline 10119 & 1 & 2 & 2 & 3 & 3 & 2 & & & 1 &
\end{tabular} \begin{tabular}{|l|l|l|l|l|l|l|l|l|l|l|l|l|l|l|r|r|r|r|r|}
\hline 101119 & 1 & 2 & 2 & 3 & 3 & 2 & 2 & 2 & 1 & 1 & 0 & 0 & 1 & 1 & 1 & 1 & 0 & 0 & 0 \\
\hline 101120 & 0 & 4 & 2 & 2 & 3 & 1 & 1 & 1 & 1 & 1 & 0 & 0 & 1 & 1 & 1 & 1 & 0 & 0 & 0 \\
\hline
\end{tabular} \begin{tabular}{|l|l|l|l|l|l|l|l|l|l|l|l|l|l|l|l|l|r|r|r|}
\hline 101121 & 1 & 3 & 2 & 3 & 3 & 2 & 1 & 1 & 1 & 1 & 0 & 0 & 1 & 1 & 1 & 1 & 0 & 0 & 0 \\
\hline 101122 & 0 & 4 & 2 & 1 & 3 & 1 & 1 & 1 & 1 & 1 & 0 & 0 & 1 & 1 & 1 & 1 & 0 & 0 \\
\hline
\end{tabular} \begin{tabular}{|l|l|l|l|l|l|l|l|l|l|l|l|l|l|l|l|r|r|r|r|}
\hline 101122 & 0 & 4 & 2 & 1 & 3 & 1 & 1 & 1 & 1 & 1 & 0 & 0 & 1 & 1 & 1 & 1 & 0 & 0 & 0 \\
\hline
\end{tabular} \begin{tabular}{|l|l|l|l|l|l|l|l|l|l|l|l|l|l|l|r|r|r|r|r|}
\hline 101123 & 0 & 4 & 2 & 3 & 3 & 1 & 2 & 1 & 1 & 1 & 0 & 0 & 1 & 1 & 1 & 1 & 0 & 0 & 0 \\
\hline
\end{tabular} \begin{tabular}{|l|l|l|l|l|l|l|l|l|l|l|l|l|l|l|r|r|r|r|r|}
\hline 101124 & 0 & 4 & 2 & 1 & 3 & 1 & 2 & 1 & 1 & 1 & 0 & 0 & 1 & 1 & 1 & 1 & 0 & 0 & 0 \\
\hline 101125 & 1 & 5 & 2 & 1 & 3 & 2
\end{tabular} \begin{tabular}{|l|l|l|l|l|l|l|l|l|l|l|l|l|l|l|r|r|r|r|r|}
\hline 101125 & 1 & 5 & 2 & 1 & 3 & 2 & 2 & 1 & 1 & 1 & 0 & 0 & 1 & 1 & 1 & 1 & 0 & 0 & 0 \\
\hline
\end{tabular} \begin{tabular}{|l|l|l|l|l|l|l|l|l|l|l|l|l|l|l|r|r|r|r|r|}
\hline 101126 & 0 & 3 & 2 & 3 & 3 & 1 & 2 & 1 & 1 & 1 & 0 & 0 & 1 & 1 & 1 & 1 & 0 & 0 & 0 \\
\hline
\end{tabular} \begin{tabular}{|l|l|l|l|l|l|l|l|l|l|l|l|l|l|l|l|l|r|r|r|}
\hline 101127 & 1 & 3 & 2 & 3 & 3 & 2 & 2 & 1 & 1 & 1 & 0 & 0 & 1 & 1 & 1 & 1 & 0 & 0 & 0 \\
\hline
\end{tabular}

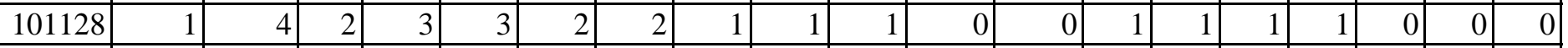

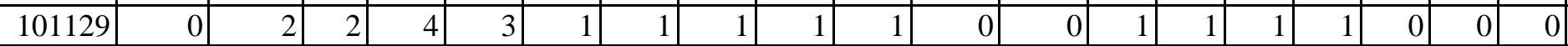

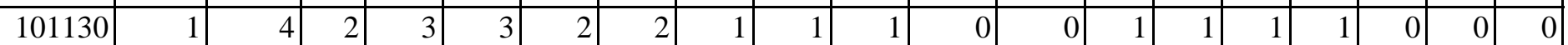
\begin{tabular}{|l|l|l|l|l|l|l|l|l|l|l|l|l|l|l|r|r|r|r|r|}
10121 & 0 & 1 & 2 & 3 & 3 & 1 & 2 & 1 & 3 & 3 & 0 & 0 & 1 & 1 & 1 & 1 & 0 & 0 & 0 \\
\hline
\end{tabular} \begin{tabular}{|l|l|l|l|l|l|l|l|l|l|l|l|l|l|l|r|r|r|r|r|}
\hline 10122 & 1 & 2 & 2 & 4 & 3 & 2 & 2 & 2 & 3 & 3 & 0 & 0 & 1 & 1 & 1 & 1 & 0 & 0 & 0 \\
\hline
\end{tabular} \begin{tabular}{|l|l|l|r|r|r|r|r|r|r|r|r|r|r|r|r|r|r|r|r|}
\hline 10123 & 0 & 2 & 2 & 2 & 3 & 1 & 2 & 2 & 3 & 3 & 0 & 0 & 1 & 1 & 1 & 1 & 0 & 0 & 0 \\
\hline
\end{tabular} \begin{tabular}{|l|l|l|l|l|l|l|l|l|l|l|l|l|l|l|r|r|r|r|r|}
\hline 10124 & 0 & 1 & 2 & 4 & 3 & 1 & 2 & 1 & 3 & 3 & 0 & 0 & 1 & 1 & 1 & 1 & 0 & 0 & 0 \\
\hline
\end{tabular} \begin{tabular}{|l|l|l|r|r|r|r|r|r|r|r|r|r|r|r|r|r|r|r|r|}
10125 & 0 & 1 & 2 & 4 & 3 & 1 & 2 & 1 & 3 & 3 & 0 & 0 & 1 & 1 & 1 & 1 & 0 & 0 & 0 \\
\hline
\end{tabular} \begin{tabular}{|r|r|r|r|r|r|r|r|r|r|r|r|r|r|r|r|r|r|r|r|}
10126 & 0 & 5 & 2 & 1 & 3 & 2 & 1 & 1 & 3 & 3 & 0 & 0 & 0 & 99 & 99 & 99 & 99 & 99 & 99 \\
\hline
\end{tabular} \begin{tabular}{|l|l|l|r|r|r|r|r|r|r|r|r|r|r|r|r|r|r|r|r|}
10127 & 1 & 5 & 2 & 1 & 3 & 2 & 1 & 1 & 3 & 3 & 0 & 0 & 1 & 1 & 3 & 1 & 1 & 0 & 1 \\
\hline 10128 & 0 & 3 & 2 & 2 & 3 & 1 & 2 & 1 & 3 & 2 & 0 & 0 & 1 & 1 & 1 & 1 & 0 & 0 & 0 \\
\hline
\end{tabular}

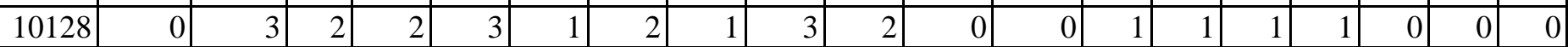

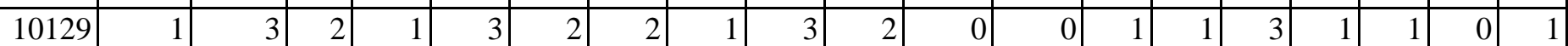

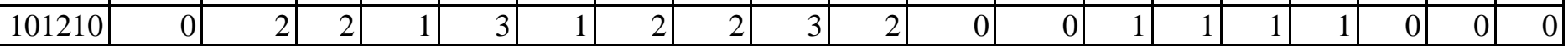
\begin{tabular}{|l|l|l|l|l|l|l|l|l|l|l|l|l|l|l|l|r|r|r|r|}
\hline 101211 & 0 & 4 & 2 & 1 & 3 & 1 & 2 & 1 & 3 & 2 & 0 & 0 & 1 & 1 & 1 & 1 & 0 & 0 & 0 \\
\hline
\end{tabular} \begin{tabular}{|l|l|l|l|l|l|l|l|l|l|l|l|l|l|l|l|l|r|r|r|}
101212 & 0 & 2 & 2 & 4 & 3 & 1 & 3 & 1 & 3 & 2 & 0 & 0 & 1 & 1 & 3 & 1 & 1 & 0 & 1 \\
\hline
\end{tabular} \begin{tabular}{|l|l|l|l|l|l|l|l|l|l|l|l|l|l|l|l|l|r|r|r|}
\hline 101213 & 1 & 5 & 2 & 2 & 3 & 2 & 2 & 1 & 3 & 2 & 0 & 0 & 1 & 1 & 3 & 1 & 1 & 0 & 1 \\
\hline
\end{tabular}

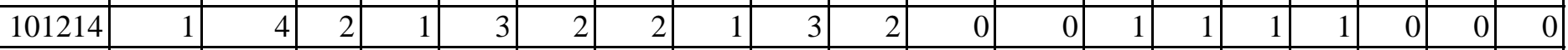
\begin{tabular}{|r|r|r|r|r|r|r|r|r|r|r|r|r|r|r|r|r|r|r|r|}
\hline 101215 & 0 & 1 & 2 & 1 & 3 & 1 & 2 & 1 & 3 & 2 & 0 & 0 & 0 & 99 & 99 & 99 & 99 & 99 & 99 \\
\hline 101216
\end{tabular}

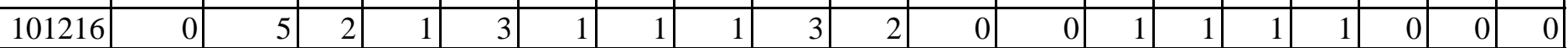
\begin{tabular}{|l|r|r|r|r|r|r|r|r|r|r|r|r|r|r|r|r|r|r|r|r|}
\hline 101217 & 1 & 2 & 2 & 2 & 3 & 5 & 2 & 1 & 3 & 2 & 0 & 0 & 1 & 1 & 1 & 1 & 0 & 0 & 0 \\
\hline
\end{tabular} \begin{tabular}{|l|r|r|r|r|r|r|r|r|r|r|r|r|r|r|r|r|r|r|r|r|}
\hline 101218 & 0 & 1 & 2 & 2 & 3 & 1 & 2 & 1 & 3 & 2 & 0 & 0 & 1 & 1 & 1 & 1 & 0 & 0 & 0 \\
\hline
\end{tabular}

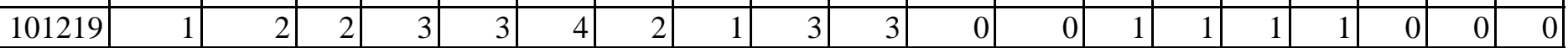

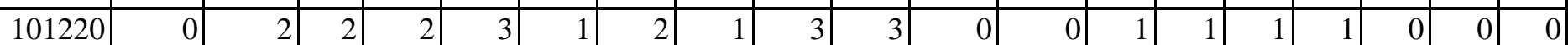
\begin{tabular}{|l|l|l|l|l|l|l|l|l|l|l|l|l|l|l|l|l|r|r|r|}
\hline 101221 & 1 & 2 & 2 & 3 & 3 & 4 & 2 & 1 & 3 & 3 & 0 & 0 & 1 & 1 & 1 & 1 & 0 & 0 & 0 \\
\hline 101222 & 1 & 2 &
\end{tabular}

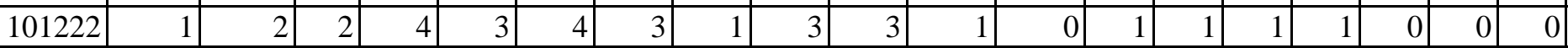

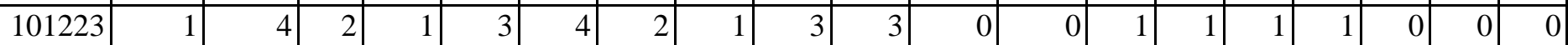

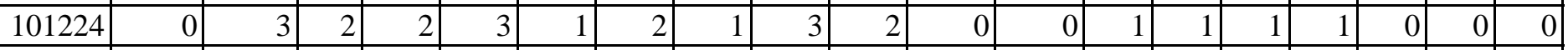

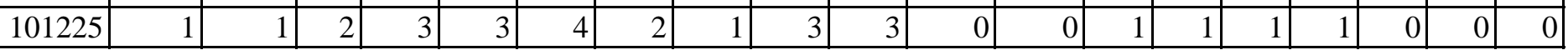




\begin{tabular}{|c|c|c|c|c|c|c|c|c|c|c|c|c|c|c|c|c|c|c|c|}
\hline 101226 & 0 & 3 & 2 & 2 & 2 & 1 & 1 & 1 & 4 & 1 & 0 & 0 & 1 & 1 & 3 & 1 & 1 & 0 & 1 \\
\hline 101227 & 1 & 2 & 2 & 3 & 2 & 4 & 2 & 1 & 3 & 1 & 0 & 0 & 1 & 1 & 1 & 1 & \begin{tabular}{l|l}
0 \\
\end{tabular} & 0 & 0 \\
\hline 101228 & 0 & 3 & 2 & 3 & 3 & 1 & 2 & 1 & 3 & 1 & 0 & 0 & 1 & 1 & 1 & 1 & 0 & 0 & 0 \\
\hline 101229 & 1 & 2 & 2 & 4 & 3 & 1 & 2 & 1 & 3 & 1 & 1 & 0 & 1 & 1 & 1 & 1 & 0 & 0 & 0 \\
\hline 101230 & 1 & 3 & 2 & 3 & 3 & 2 & 3 & 1 & 3 & 1 & 0 & 0 & 1 & 1 & 1 & 1 & 0 & 0 & 0 \\
\hline 101231 & 1 & 5 & 2 & 1 & 3 & 2 & 1 & 1 & 3 & 1 & 0 & 0 & 0 & 99 & 99 & 99 & 99 & 99 & 99 \\
\hline 101232 & 1 & 5 & 2 & 1 & 3 & 2 & 1 & 1 & 3 & 2 & 0 & 0 & 1 & 1 & 1 & 1 & \begin{tabular}{l|l}
0 \\
\end{tabular} & 0 & 0 \\
\hline 101233 & 1 & 2 & 2 & 1 & 3 & 2 & 1 & 1 & 3 & 1 & 0 & 0 & 1 & 1 & 1 & 1 & 0 & 0 & 0 \\
\hline 101234 & 1 & 3 & 2 & 2 & 3 & 2 & 1 & 2 & 3 & 3 & 0 & 0 & 1 & 1 & 1 & 1 & 0 & 0 & 0 \\
\hline 101235 & 1 & 5 & 2 & 1 & 3 & 2 & 1 & 1 & 3 & 3 & 0 & 0 & 1 & 1 & 1 & 1 & 0 & 0 & 0 \\
\hline 10131 & 0 & 3 & 2 & 3 & 3 & 1 & 2 & 1 & 4 & 1 & 0 & 0 & 1 & 1 & 1 & 1 & 0 & 0 & 0 \\
\hline 10132 & 1 & 1 & 2 & 4 & 3 & 5 & 2 & 1 & 4 & 1 & 0 & 0 & 1 & 1 & 1 & 1 & 0 & 0 & 0 \\
\hline 10133 & 1 & 3 & 2 & 4 & 3 & 5 & 2 & 1 & 4 & 1 & 0 & 0 & 1 & 1 & 1 & 1 & 0 & 0 & 0 \\
\hline 10134 & 0 & 2 & 2 & 4 & 3 & 1 & 2 & 1 & 4 & 1 & 1 & 0 & 1 & 1 & 1 & 1 & 0 & 0 & 0 \\
\hline 10135 & 0 & 1 & 2 & 4 & 3 & 1 & 2 & 1 & 4 & 1 & 0 & 0 & 1 & 1 & 1 & 1 & 0 & 0 & 0 \\
\hline 10136 & 0 & 3 & 2 & 4 & 3 & 1 & 2 & 1 & 4 & 1 & 0 & 0 & 1 & 1 & 1 & 1 & 0 & 0 & 0 \\
\hline 10137 & 1 & 2 & 2 & 4 & 3 & 5 & 3 & 2 & 4 & 2 & 0 & 0 & 1 & 1 & 1 & 1 & 0 & 0 & 0 \\
\hline 10138 & 0 & 2 & 2 & 4 & 3 & 1 & 2 & 1 & 4 & 2 & 1 & 0 & 1 & 1 & 1 & 1 & 0 & 0 & 0 \\
\hline 10139 & 0 & 2 & 2 & 3 & 3 & 1 & 2 & 1 & 4 & 2 & 1 & 0 & 1 & 1 & 1 & 1 & 0 & 0 & 0 \\
\hline 101310 & 1 & 2 & 2 & 4 & 3 & 5 & 2 & 1 & 4 & 2 & 0 & 0 & 1 & 1 & 1 & 1 & 0 & 0 & 0 \\
\hline 101311 & 1 & 1 & 2 & 4 & 3 & 5 & 2 & 1 & 4 & 2 & 0 & 0 & 1 & 1 & 1 & 1 & 0 & 0 & 0 \\
\hline 101312 & 0 & 2 & 2 & 4 & 3 & 1 & 2 & 1 & 4 & 2 & 0 & 0 & 1 & 1 & 1 & 1 & 0 & 0 & 0 \\
\hline 101313 & 0 & 3 & 2 & 1 & 3 & 1 & 1 & 1 & 4 & 2 & 0 & 0 & 1 & 1 & 1 & 1 & 0 & \begin{tabular}{l|l}
0 \\
\end{tabular} & 0 \\
\hline 101314 & 1 & 2 & 2 & 4 & 3 & 2 & 2 & 1 & 4 & 2 & 0 & 0 & 1 & 1 & 1 & 1 & 0 & 0 & 0 \\
\hline 101315 & 1 & 1 & 2 & 3 & 3 & 2 & 1 & 1 & 4 & 2 & 0 & 0 & 1 & 1 & 1 & 1 & 0 & 0 & 0 \\
\hline 101316 & 0 & 3 & 2 & 3 & 3 & 1 & 2 & 1 & 4 & 2 & 0 & 0 & 1 & 1 & 1 & 1 & 0 & 0 & 0 \\
\hline 101317 & 0 & 2 & 2 & 3 & 3 & 1 & 2 & 1 & 4 & 2 & 0 & 0 & 1 & 1 & 1 & 1 & 0 & 0 & 0 \\
\hline 101318 & 0 & 4 & 2 & 4 & 3 & 1 & 2 & 2 & 4 & 2 & 0 & 0 & 1 & 1 & 1 & 1 & 0 & 0 & 0 \\
\hline 101319 & 1 & 3 & 2 & 3 & 3 & 3 & 3 & 1 & 4 & 2 & 0 & 0 & 1 & 1 & 1 & 1 & 0 & \begin{tabular}{l|l}
0 \\
\end{tabular} & 0 \\
\hline 101320 & 1 & 5 & 2 & 2 & 3 & 2 & 2 & 1 & 4 & 2 & 0 & 0 & 1 & 1 & 1 & 1 & 0 & 0 & 0 \\
\hline 101321 & 0 & 4 & 2 & 2 & 3 & 1 & 2 & 1 & 4 & 2 & 0 & 0 & 1 & 1 & 1 & 1 & 0 & 0 & 0 \\
\hline 101322 & 1 & 2 & 2 & 3 & 3 & 2 & 2 & 1 & 4 & 2 & 0 & 0 & 1 & 1 & 1 & 1 & 0 & 0 & 0 \\
\hline 101323 & 0 & 2 & 2 & 1 & 3 & 2 & 2 & 2 & 4 & 2 & 0 & 0 & 1 & 1 & 1 & 1 & 0 & 0 & 0 \\
\hline 101324 & 1 & 4 & 2 & 2 & 3 & 2 & 2 & 1 & 4 & 2 & 0 & 0 & 1 & 1 & 1 & 1 & 0 & 0 & 0 \\
\hline 101325 & 1 & 3 & 2 & 3 & 3 & 1 & 2 & 1 & 4 & 2 & 0 & 0 & 1 & 1 & 1 & 1 & 0 & 0 & 0 \\
\hline 101326 & 0 & 1 & 2 & 1 & 3 & 1 & 2 & 1 & 4 & 2 & 0 & 0 & 1 & 1 & 1 & 1 & 0 & 0 & 0 \\
\hline 101327 & 1 & 1 & 2 & 3 & 3 & 2 & 2 & 1 & 4 & 2 & 0 & 0 & 1 & 1 & 1 & 1 & 0 & 0 & 0 \\
\hline 101328 & 1 & 5 & 2 & 1 & 3 & 2 & 2 & 1 & 4 & 2 & 0 & 0 & 1 & 1 & 1 & 1 & 0 & 0 & 0 \\
\hline 101329 & 0 & 1 & 2 & 4 & 3 & 1 & 2 & 1 & 4 & 2 & 0 & 0 & 1 & 1 & 1 & 1 & 0 & 0 & 0 \\
\hline 101330 & 1 & 5 & 2 & 3 & 3 & 2 & 2 & 1 & 4 & 2 & 0 & 0 & 1 & 1 & 1 & 1 & 0 & 0 & 0 \\
\hline 10141 & 1 & 2 & 2 & 5 & 3 & 5 & 3 & 1 & 4 & 1 & 0 & 0 & 1 & 1 & 1 & 1 & 0 & 0 & 0 \\
\hline 10142 & 0 & 2 & 2 & 4 & 3 & 1 & 2 & 1 & 4 & 1 & 0 & 0 & 1 & 1 & 3 & 1 & 1 & 0 & 1 \\
\hline 10143 & 1 & 5 & 2 & 2 & 3 & 5 & 2 & 1 & 4 & 1 & 1 & 1 & 1 & 1 & 3 & 2 & 1 & 0 & 1 \\
\hline 10144 & 0 & 1 & 2 & 4 & 3 & 5 & 2 & 1 & 4 & 1 & 0 & 0 & 1 & 1 & 3 & 1 & 1 & 0 & 1 \\
\hline 10145 & 1 & 4 & 2 & 1 & 3 & 2 & 1 & 2 & 4 & 1 & 0 & 0 & 0 & 99 & 99 & 99 & 99 & 99 & 99 \\
\hline 10146 & 0 & 5 & 2 & 2 & 2 & 1 & 1 & 2 & 4 & 1 & 0 & 0 & 0 & 99 & 99 & 99 & 99 & \begin{tabular}{l|l|}
99 \\
\end{tabular} & 99 \\
\hline 10147 & 1 & 2 & 2 & 4 & 3 & 5 & 2 & 2 & 4 & 1 & 0 & 0 & 1 & 1 & 2 & 1 & 1 & 0 & 1 \\
\hline 10148 & 1 & 4 & 2 & 1 & 3 & 2 & 2 & 2 & 4 & 1 & 0 & 0 & 1 & 1 & 3 & 6 & 1 & 1 & 1 \\
\hline 10149 & 0 & 3 & 2 & 2 & 3 & 1 & 3 & 2 & 4 & 2 & 0 & 0 & 1 & 1 & 1 & 1 & 0 & 0 & 0 \\
\hline 101410 & 0 & 3 & 2 & 2 & 2 & 1 & 2 & 2 & 4 & 2 & 0 & 0 & 1 & 0 & 5 & 5 & 1 & 1 & 1 \\
\hline 101411 & 1 & 5 & 2 & 1 & 3 & 5 & 1 & 1 & 4 & 2 & 0 & 0 & 0 & 99 & 99 & 99 & 99 & \begin{tabular}{l|l|}
99 \\
\end{tabular} & 99 \\
\hline 101412 & 1 & 3 & 2 & 2 & 3 & 2 & 2 & 1 & 4 & 2 & 0 & 0 & 1 & 1 & 5 & 5 & 1 & 1 & 1 \\
\hline 101413 & 1 & 2 & 2 & 4 & 3 & 2 & 2 & 1 & 4 & 2 & 0 & 0 & 1 & 1 & 1 & 1 & 0 & 0 & 0 \\
\hline 101414 & 1 & 3 & 2 & 3 & 3 & 2 & 1 & 1 & 4 & 2 & 0 & 0 & 0 & 99 & 99 & 99 & 99 & 99 & 99 \\
\hline 101415 & 1 & 4 & 2 & 1 & 3 & 2 & 1 & 1 & 4 & 2 & 0 & 0 & 0 & 99 & 99 & 99 & 99 & 99 & 99 \\
\hline 101416 & 0 & 4 & 2 & 1 & 3 & 1 & 1 & 1 & 4 & 2 & 0 & 0 & 0 & 99 & 99 & 99 & 99 & (99 & 99 \\
\hline
\end{tabular}




\begin{tabular}{|c|c|c|c|c|c|c|c|c|c|c|c|c|c|c|c|c|c|c|c|}
\hline 101417 & 1 & 3 & 2 & 2 & 3 & 2 & 2 & 1 & 4 & 2 & 0 & 0 & 1 & 1 & 2 & 1 & 1 & 0 & 1 \\
\hline 101418 & 1 & 4 & 2 & 2 & 3 & 2 & 1 & 1 & 4 & 2 & 0 & 0 & 1 & 1 & 3 & 1 & 1 & 0 & 1 \\
\hline 101419 & 1 & 3 & 2 & 2 & 3 & 2 & 2 & 1 & 4 & 2 & 0 & 0 & 1 & 1 & 2 & 2 & 1 & 0 & 1 \\
\hline 101420 & 0 & 2 & 2 & 3 & 3 & 1 & 2 & 2 & 4 & 2 & 0 & 0 & 1 & 1 & 3 & 1 & 1 & 0 & 1 \\
\hline 101421 & 1 & 4 & 2 & 2 & 3 & 2 & 2 & 1 & 4 & 2 & 0 & 0 & 0 & 99 & 99 & 99 & 99 & 99 & 99 \\
\hline 101422 & 0 & 3 & 2 & 1 & 3 & 1 & 1 & 1 & 4 & 2 & 0 & 0 & 0 & 99 & 99 & 99 & 99 & 99 & 99 \\
\hline 101423 & 1 & 4 & 2 & 2 & 3 & 2 & 2 & 1 & 4 & 2 & 0 & 0 & 1 & 1 & 2 & 1 & 1 & 0 & 1 \\
\hline 101424 & 0 & 1 & 2 & 4 & 2 & 1 & 2 & 1 & 4 & 1 & 0 & 0 & 1 & 1 & 2 & 1 & 1 & 0 & 1 \\
\hline 101425 & 1 & 1 & 2 & 4 & 3 & 4 & 2 & 1 & 4 & 1 & 0 & 0 & 1 & 1 & 3 & 1 & 1 & 0 & 1 \\
\hline 101426 & 0 & 1 & 2 & 3 & 3 & 1 & 1 & 1 & 4 & 2 & 0 & 0 & 1 & 1 & 1 & 1 & 0 & 0 & 0 \\
\hline 101427 & 0 & 3 & 2 & 3 & 3 & 1 & 2 & 1 & 4 & 2 & 0 & 0 & 1 & 1 & 2 & 1 & 1 & 0 & 1 \\
\hline 101428 & 0 & 4 & 2 & 1 & 3 & 1 & 2 & 2 & 4 & 2 & 0 & 0 & 1 & 1 & 2 & 1 & 1 & 0 & 1 \\
\hline 101429 & 1 & 2 & 2 & 3 & 3 & 2 & 2 & 1 & 4 & 2 & 0 & 0 & 1 & 1 & 3 & 1 & 1 & 0 & 1 \\
\hline 101430 & 0 & 3 & 2 & 2 & 3 & 1 & 2 & 1 & 4 & 2 & 0 & 0 & 1 & 1 & 2 & 1 & 1 & 0 & 1 \\
\hline 101431 & 1 & 4 & 2 & 2 & 3 & 2 & 2 & 2 & 4 & 2 & 0 & 0 & 1 & 1 & 2 & 1 & 1 & 0 & 1 \\
\hline 101432 & 1 & 4 & 2 & 1 & 3 & 1 & 2 & 1 & 4 & 2 & 0 & 0 & 1 & 1 & 3 & 1 & 1 & 0 & 1 \\
\hline 101433 & 0 & 1 & 2 & 3 & 3 & 1 & 2 & 1 & 4 & 2 & 0 & 0 & 1 & 1 & 2 & 1 & 1 & 0 & 1 \\
\hline 101434 & 1 & 2 & 2 & 3 & 4 & 2 & 1 & 1 & 4 & 2 & 0 & 0 & 1 & 1 & 1 & 1 & 0 & 0 & 0 \\
\hline 101435 & 1 & 2 & 2 & 3 & 3 & 4 & 2 & 1 & 4 & 2 & 0 & 0 & 1 & 1 & 2 & 1 & 1 & 0 & 1 \\
\hline 10151 & 0 & 2 & 2 & 4 & 3 & 1 & 2 & 2 & 4 & 2 & 0 & 0 & 1 & 1 & 2 & 1 & 1 & 0 & 1 \\
\hline 10152 & 1 & 4 & 2 & 4 & 3 & 4 & 2 & 1 & 4 & 2 & 0 & 0 & 1 & 1 & 3 & 2 & 1 & 0 & 1 \\
\hline 10153 & 0 & 2 & 2 & 3 & 3 & 1 & 2 & 1 & 4 & 2 & 0 & 0 & 1 & 1 & 2 & 1 & 1 & 0 & 1 \\
\hline 10154 & 0 & 1 & 2 & 4 & 3 & 5 & 2 & 2 & 4 & 2 & 1 & 0 & 1 & 1 & 2 & 2 & 1 & 0 & 1 \\
\hline 10155 & 1 & 3 & 2 & 3 & 3 & 4 & 2 & 1 & 4 & 2 & 0 & 0 & 1 & 1 & 1 & 1 & 0 & 0 & 0 \\
\hline 10156 & 1 & 3 & 2 & 3 & 3 & 5 & 2 & 1 & 4 & 2 & 0 & 0 & 1 & 1 & 2 & 1 & 1 & 0 & 1 \\
\hline 10157 & 0 & 1 & 2 & 4 & 3 & 1 & 2 & 1 & 1 & 1 & 0 & 0 & 1 & 1 & 2 & 1 & 1 & 0 & 1 \\
\hline 10158 & 0 & 2 & 2 & 4 & 3 & 1 & 2 & 1 & 1 & 1 & 0 & 0 & 1 & 1 & 3 & 1 & 1 & 0 & 1 \\
\hline 10159 & 1 & 5 & 2 & 4 & 3 & 5 & 3 & 1 & 4 & 3 & 1 & 1 & 1 & 1 & 2 & 1 & 1 & 0 & 1 \\
\hline 101510 & 1 & 1 & 2 & 4 & 3 & 4 & 2 & 1 & 1 & 1 & 0 & 0 & 1 & 1 & 2 & 1 & 1 & 0 & 1 \\
\hline 101511 & 0 & 4 & 2 & 4 & 3 & 5 & 3 & 2 & 1 & 1 & 1 & 1 & 1 & 1 & 3 & 1 & 1 & 0 & 1 \\
\hline 101512 & 0 & 2 & 2 & 5 & 3 & 1 & 1 & 1 & 1 & 1 & 0 & 0 & 1 & 1 & 2 & 4 & 1 & 1 & 1 \\
\hline 101513 & 1 & 3 & 2 & 4 & 3 & 4 & 2 & 2 & 1 & 2 & 1 & 0 & 1 & 1 & 2 & 1 & 1 & 0 & 1 \\
\hline 101514 & 1 & 5 & 2 & 3 & 3 & 4 & 2 & 1 & 1 & 2 & 0 & 0 & 1 & 1 & 1 & 1 & 0 & 0 & 0 \\
\hline 101515 & 0 & 1 & 2 & 4 & 3 & 1 & 2 & 1 & 1 & 1 & 0 & 0 & 1 & 1 & 2 & 1 & 1 & 0 & 1 \\
\hline 101516 & 1 & 2 & 2 & 2 & 3 & 4 & 2 & 1 & 1 & 1 & 0 & 0 & 1 & 1 & 2 & 2 & 1 & 0 & 1 \\
\hline 101517 & 0 & 2 & 2 & 3 & 2 & 1 & 2 & 2 & 1 & 1 & 0 & 0 & 1 & 1 & 3 & 1 & 1 & 0 & 1 \\
\hline 101518 & 1 & 3 & 2 & 4 & 3 & 4 & 2 & 1 & 1 & 1 & 0 & 0 & 1 & 1 & 2 & 1 & 1 & 0 & 1 \\
\hline 101519 & 0 & 2 & 2 & 4 & 3 & 1 & 2 & 2 & 1 & 1 & 0 & 0 & 1 & 1 & 2 & 1 & 1 & 0 & 1 \\
\hline 101520 & 1 & 4 & 2 & 4 & 3 & 4 & 3 & 2 & 1 & 2 & 1 & 1 & 1 & 1 & 2 & 1 & 1 & 0 & 1 \\
\hline 101521 & 0 & 5 & 2 & 2 & 3 & 1 & 2 & 1 & 1 & 2 & 0 & 0 & 1 & 1 & 3 & 1 & 1 & 0 & 1 \\
\hline 101522 & 1 & 5 & 2 & 3 & 3 & 5 & 2 & 1 & 1 & 2 & 1 & 1 & 1 & 1 & 2 & 1 & 1 & 0 & 1 \\
\hline 101523 & 1 & 4 & 2 & 4 & 3 & 5 & 2 & 1 & 1 & 1 & 1 & 1 & 1 & 1 & 2 & 2 & 1 & 0 & 1 \\
\hline 101524 & 0 & 3 & 2 & 4 & 3 & 5 & 3 & 1 & 1 & 1 & 1 & 1 & 1 & 1 & 3 & 2 & 1 & 0 & 1 \\
\hline 101525 & 1 & 2 & 2 & 4 & 3 & 5 & 2 & 2 & 1 & 1 & 1 & 1 & 1 & 1 & 2 & 1 & 1 & 0 & 1 \\
\hline 101526 & 1 & 2 & 2 & 4 & 3 & 5 & 2 & 1 & 1 & 1 & 1 & 1 & 1 & 1 & 2 & 1 & 1 & 0 & 1 \\
\hline 101527 & 1 & 3 & 2 & 4 & 3 & 5 & 2 & 1 & 1 & 1 & 0 & 0 & 1 & 1 & 2 & 1 & 1 & 0 & 1 \\
\hline 101528 & 1 & 4 & 2 & 2 & 3 & 2 & 2 & 1 & 1 & 1 & 1 & 1 & 1 & 1 & 2 & 1 & 1 & 0 & 1 \\
\hline 101529 & 0 & 3 & 2 & 3 & 3 & 1 & 2 & 1 & 1 & 1 & 0 & 0 & 1 & 1 & 3 & 1 & 1 & 0 & 1 \\
\hline 101530 & 0 & 3 & 2 & 4 & 3 & 1 & 2 & 1 & 1 & 1 & 0 & 0 & 1 & 1 & 2 & 1 & 1 & 0 & 1 \\
\hline 10161 & 0 & 1 & 2 & 4 & 3 & 1 & 2 & 1 & 1 & 1 & 0 & 0 & 1 & 1 & 2 & 1 & 1 & 0 & 1 \\
\hline 10162 & 0 & 1 & 2 & 2 & 3 & 1 & 2 & 1 & 2 & 2 & 1 & 0 & 1 & 1 & 2 & 1 & 1 & 0 & 1 \\
\hline 10163 & 0 & 2 & 2 & 4 & 3 & 1 & 3 & 1 & 1 & 2 & 0 & 0 & 1 & 1 & 3 & 1 & 1 & 0 & 1 \\
\hline 10164 & 1 & 4 & 2 & 5 & 3 & 5 & 3 & 2 & 1 & 2 & 1 & 1 & 1 & 1 & 1 & 6 & 0 & 1 & 1 \\
\hline 10165 & 1 & 2 & 2 & 2 & 3 & 2 & 2 & 1 & 1 & 2 & 0 & 0 & 1 & 1 & 2 & 1 & 1 & 0 & 1 \\
\hline 10166 & 1 & 5 & 2 & 2 & 3 & 2 & 2 & 1 & 1 & 2 & 1 & 1 & 1 & 1 & 2 & 1 & 1 & 0 & 1 \\
\hline 10167 & 1 & 4 & 2 & 2 & 3 & 4 & 2 & 2 & 1 & 1 & 0 & 0 & 1 & 1 & 2 & 1 & 1 & 0 & 1 \\
\hline
\end{tabular}




\begin{tabular}{|c|c|c|c|c|c|c|c|c|c|c|c|c|c|c|c|c|c|c|c|}
\hline 10168 & 1 & 1 & 2 & 2 & 3 & 2 & 2 & 1 & 1 & 1 & 0 & 0 & 1 & 1 & 3 & 1 & 1 & 0 & 1 \\
\hline 10169 & 0 & 3 & 2 & 2 & 3 & 1 & 2 & 1 & 1 & 1 & 0 & 0 & 1 & 1 & 3 & 2 & 1 & 0 & 1 \\
\hline 101610 & 0 & 3 & 2 & 1 & 3 & 1 & 2 & 2 & 1 & 1 & 0 & 0 & 1 & 1 & 2 & 1 & 1 & \begin{tabular}{l|l}
0 \\
\end{tabular} & 1 \\
\hline 101611 & 0 & 1 & 2 & 2 & 3 & 1 & 2 & 2 & 1 & 1 & 0 & 0 & 1 & 1 & 2 & 6 & 1 & 1 & 1 \\
\hline 101612 & 0 & 4 & 2 & 2 & 3 & 1 & 2 & 1 & 1 & 1 & 0 & 0 & 1 & 1 & 4 & 6 & 1 & 1 & 1 \\
\hline 101613 & 1 & 3 & 2 & 2 & 3 & 4 & 2 & 1 & 1 & 1 & 0 & 0 & 1 & 1 & 2 & 1 & 1 & 0 & 1 \\
\hline 101614 & 0 & 3 & 2 & 2 & 3 & 1 & 3 & 2 & 1 & 1 & 0 & 0 & 1 & 1 & 2 & 1 & 1 & \begin{tabular}{l|l}
0 \\
\end{tabular} & 1 \\
\hline 101615 & 0 & 4 & 2 & 2 & 3 & 1 & 2 & 1 & 1 & 1 & 0 & 0 & 1 & 1 & 3 & 1 & 1 & 0 & 1 \\
\hline 101616 & 0 & 1 & 2 & 4 & 3 & 1 & 2 & 1 & 1 & 1 & 0 & 0 & 1 & 1 & 2 & 1 & 1 & 0 & 1 \\
\hline 101617 & 0 & 3 & 2 & 1 & 3 & 1 & 2 & 2 & 1 & 1 & 0 & 0 & 1 & 1 & 2 & 1 & 1 & 0 & 1 \\
\hline 101618 & 1 & 5 & 2 & 2 & 3 & 2 & 1 & 1 & 1 & 1 & 0 & 0 & 1 & 1 & 3 & 2 & 1 & 0 & 1 \\
\hline 101619 & 1 & 4 & 2 & 2 & 3 & 4 & 2 & 2 & 1 & 1 & 0 & 0 & 1 & 1 & 3 & 6 & 1 & 1 & 1 \\
\hline 101620 & 0 & 1 & 2 & 3 & 3 & 1 & 2 & 2 & 1 & 1 & 0 & 0 & 1 & 1 & 3 & 1 & 1 & 0 & 1 \\
\hline 101621 & 1 & 4 & 2 & 2 & 3 & 4 & 2 & 1 & 1 & 1 & 0 & 0 & 1 & 1 & 2 & 1 & 1 & 0 & 1 \\
\hline 101622 & 0 & 2 & 2 & 3 & 3 & 1 & 2 & 1 & 1 & 1 & 0 & 0 & 1 & 1 & 2 & 1 & 1 & 0 & 1 \\
\hline 101623 & 1 & 1 & 2 & 2 & 3 & 4 & 2 & 1 & 1 & 1 & 0 & 0 & 1 & 1 & 3 & 1 & 1 & 0 & 1 \\
\hline 101624 & 0 & 5 & 2 & 2 & 3 & 1 & 1 & 1 & 1 & 1 & 0 & 0 & 1 & 1 & 2 & 6 & 1 & 1 & 1 \\
\hline 101625 & 1 & 2 & 2 & 3 & 1 & 2 & 2 & 1 & 1 & 1 & 0 & 0 & 1 & 1 & 3 & 1 & 1 & 0 & 1 \\
\hline 101626 & 1 & 4 & 2 & 2 & 3 & 4 & 2 & 2 & 1 & 1 & 0 & 0 & 1 & 1 & 2 & 1 & 1 & 0 & 1 \\
\hline 101627 & 1 & 3 & 2 & 2 & 3 & 4 & 2 & 1 & 1 & 1 & 0 & 0 & 1 & 1 & 2 & 1 & 1 & 0 & 1 \\
\hline 101628 & 1 & 2 & 2 & 2 & 3 & 4 & 2 & 2 & 1 & 1 & 0 & 0 & 1 & 1 & 2 & 6 & 1 & 1 & 1 \\
\hline 101629 & 1 & 3 & 2 & 3 & 3 & 4 & 2 & 1 & 1 & 1 & 0 & 0 & 1 & 1 & 2 & 1 & 1 & 0 & 1 \\
\hline 101630 & 0 & 4 & 2 & 2 & 3 & 1 & 2 & 1 & 1 & 1 & 0 & 0 & 1 & 1 & 2 & 1 & 1 & 0 & 1 \\
\hline 10271 & 0 & 3 & 2 & 1 & 3 & 1 & 2 & 2 & 1 & 1 & 0 & 0 & 1 & 1 & 3 & 4 & 1 & 1 & 1 \\
\hline 10272 & 0 & 1 & 2 & 4 & 3 & 1 & 2 & 2 & 1 & 1 & 0 & 0 & 1 & 1 & 2 & 1 & 1 & 0 & 1 \\
\hline 10273 & 0 & 2 & 2 & 2 & 3 & 1 & 2 & 2 & 1 & 3 & 0 & 0 & 1 & 1 & 3 & 1 & 1 & 0 & 1 \\
\hline 10274 & 1 & 2 & 2 & 2 & 3 & 4 & 2 & 2 & 1 & 3 & 0 & 0 & 1 & 1 & 2 & 1 & 1 & 0 & 1 \\
\hline 10275 & 0 & 4 & 2 & 2 & 3 & 1 & 1 & 1 & 1 & 3 & 0 & 0 & 1 & 1 & 1 & 5 & 0 & 1 & 1 \\
\hline 10276 & 1 & 2 & 2 & 1 & 3 & 2 & 2 & 1 & 1 & 3 & 0 & 0 & 1 & 0 & 5 & 5 & 1 & 1 & 1 \\
\hline 10277 & 0 & 1 & 2 & 3 & 3 & 1 & 2 & 2 & 1 & 3 & 0 & 0 & 1 & 1 & 3 & 6 & 1 & 1 & 1 \\
\hline 10278 & 0 & 5 & 2 & 1 & 3 & 1 & 1 & 1 & 1 & 1 & 0 & 0 & 0 & 99 & 99 & 99 & 99 & 99 & 99 \\
\hline 10279 & 1 & 4 & 2 & 2 & 3 & 2 & 2 & 2 & 1 & 3 & 0 & 0 & 1 & 1 & 1 & 1 & 0 & 0 & 0 \\
\hline 102710 & 1 & 3 & 2 & 4 & 3 & 2 & 2 & 1 & 1 & 3 & 0 & 0 & 1 & 1 & 2 & 1 & 1 & \begin{tabular}{l|l}
0 \\
\end{tabular} & 1 \\
\hline 102711 & 1 & 4 & 2 & 1 & 3 & 2 & 2 & 1 & 1 & 3 & 0 & 0 & 1 & 1 & 4 & 4 & 1 & 1 & 1 \\
\hline 102712 & 0 & 2 & 2 & 4 & 3 & 1 & 2 & 1 & 1 & 3 & 0 & 0 & 1 & 1 & 1 & 1 & 0 & 0 & 0 \\
\hline 102713 & 0 & 4 & 2 & 2 & 3 & 1 & 2 & 1 & 1 & 3 & 0 & 0 & 0 & 99 & 99 & 99 & 99 & 99 & 99 \\
\hline 102714 & 0 & 3 & 2 & 3 & 3 & 1 & 1 & 1 & 1 & 3 & 0 & 0 & 1 & 1 & 3 & 4 & 1 & 1 & 1 \\
\hline 102715 & 1 & 5 & 2 & 1 & 3 & 2 & 1 & 1 & 1 & 3 & 0 & 0 & 0 & 99 & 99 & 99 & 99 & 99 & 99 \\
\hline 102716 & 1 & 4 & 2 & 2 & 3 & 2 & 1 & 1 & 1 & 3 & 0 & 0 & 1 & 1 & 3 & 6 & 1 & 1 & 1 \\
\hline 102717 & 0 & 4 & 2 & 2 & 3 & 1 & 1 & 1 & 1 & 3 & 0 & 0 & 0 & 99 & 99 & 99 & 99 & 99 & 99 \\
\hline 102718 & 1 & 3 & 2 & 2 & 3 & 2 & 1 & 1 & 1 & 3 & 0 & 0 & 1 & 1 & 1 & 1 & 0 & 0 & 0 \\
\hline 102719 & 0 & 4 & 2 & 1 & 3 & 2 & 1 & 1 & 1 & 3 & 0 & 0 & 1 & 1 & 2 & 5 & 1 & 1 & 1 \\
\hline 102720 & 1 & 3 & 2 & 3 & 3 & 4 & 2 & 1 & 1 & 3 & 0 & 0 & 1 & 1 & 2 & 6 & 1 & 1 & 1 \\
\hline 102721 & 1 & 4 & 2 & 2 & 3 & 2 & 2 & 1 & 1 & 3 & 0 & 0 & 1 & 1 & 1 & 1 & 0 & 0 & 0 \\
\hline 102722 & 0 & 4 & 2 & 1 & 3 & 1 & 1 & 1 & 1 & 3 & 0 & 0 & 0 & 99 & 99 & 99 & 99 & \begin{tabular}{l|l|}
99 \\
\end{tabular} & 99 \\
\hline 102723 & 0 & 2 & 2 & 2 & 3 & 1 & 1 & 1 & 1 & 3 & 0 & 0 & 1 & 1 & 3 & 5 & 1 & 1 & 1 \\
\hline 102724 & 1 & 3 & 2 & 1 & 3 & 2 & 2 & 1 & 1 & 3 & 0 & 0 & 1 & 1 & 2 & 1 & 1 & 0 & 1 \\
\hline 102725 & 0 & 2 & 2 & 2 & 3 & 1 & 1 & 2 & 1 & 3 & 0 & 0 & 1 & 1 & 4 & 1 & 1 & 0 & 1 \\
\hline 102726 & 1 & 3 & 2 & 2 & 3 & 2 & 2 & 1 & 1 & 3 & 0 & 0 & 1 & 1 & 3 & 7 & 1 & 1 & 1 \\
\hline 102727 & 1 & 2 & 2 & 2 & 3 & 2 & 2 & 1 & 1 & 2 & 0 & 0 & 1 & 1 & 1 & 1 & 0 & 0 & 0 \\
\hline 102728 & 1 & 2 & 2 & 4 & 3 & 2 & 2 & 1 & 1 & 2 & 0 & 0 & 1 & 1 & 1 & 1 & 0 & 0 & 0 \\
\hline 102729 & 1 & 2 & 2 & 3 & 3 & 4 & 2 & 2 & 1 & 3 & 0 & 0 & 1 & 1 & 2 & 6 & 1 & 1 & \\
\hline 102730 & 1 & 3 & 2 & 2 & 3 & 4 & 2 & 1 & 1 & 3 & 0 & 0 & 1 & 1 & 2 & 6 & 1 & 1 & 1 \\
\hline 10281 & 1 & 3 & 2 & 2 & 3 & 2 & 2 & 1 & 1 & 4 & 0 & 0 & 1 & 1 & 2 & 6 & 1 & 1 & 1 \\
\hline 10282 & 1 & 4 & 2 & 2 & 3 & 2 & 1 & 1 & 1 & 1 & 0 & 0 & 0 & 99 & 99 & 99 & 99 & 99 & 99 \\
\hline 10283 & 0 & 4 & 2 & 1 & 3 & 1 & 1 & 1 & 1 & 4 & 0 & 0 & 11 & 1 & 3 & 5 & 1 & 1 & \\
\hline
\end{tabular}




\begin{tabular}{|c|c|c|c|c|c|c|c|c|c|c|c|c|c|c|c|c|c|c|c|}
\hline 10284 & 1 & 3 & 2 & 1 & 3 & 1 & 2 & 1 & 1 & 4 & 0 & 0 & 1 & 1 & 2 & 5 & 1 & 1 & 1 \\
\hline 10285 & 1 & 4 & 2 & 2 & 3 & 2 & 2 & 1 & 1 & 4 & 0 & 0 & 1 & 1 & 1 & 4 & 0 & 1 & 1 \\
\hline 10286 & 0 & 3 & 2 & 2 & 3 & 1 & 1 & 1 & 1 & 4 & 0 & 0 & 1 & 1 & 2 & 4 & 1 & 1 & 1 \\
\hline 10287 & \begin{tabular}{l|}
0 \\
\end{tabular} & 5 & 2 & 1 & 3 & 1 & 1 & 1 & 1 & 4 & 0 & 0 & 0 & 99 & 99 & 99 & 99 & 99 & 99 \\
\hline 10288 & 1 & 5 & 2 & 1 & 3 & 2 & 1 & 1 & 1 & 4 & 0 & 0 & 0 & 99 & 99 & 99 & 99 & 99 & 99 \\
\hline 10289 & 1 & 1 & 2 & 3 & 3 & 2 & 1 & 1 & 1 & 4 & 0 & 0 & 1 & 1 & 3 & 1 & 1 & 0 & 1 \\
\hline 102810 & 0 & 3 & 2 & 2 & 3 & 1 & 1 & 1 & 1 & 4 & 0 & 0 & 1 & 1 & 2 & 1 & 1 & 0 & 1 \\
\hline 102811 & 1 & 3 & 2 & 3 & 3 & 2 & 2 & 1 & 1 & 4 & 0 & 0 & 1 & 1 & 1 & 2 & 0 & 0 & 0 \\
\hline 102812 & 1 & 3 & 2 & 3 & 3 & 2 & 2 & 1 & 1 & 4 & 0 & 0 & 1 & 1 & 1 & 1 & 0 & 0 & 0 \\
\hline 102813 & 1 & 4 & 2 & 2 & 3 & 2 & 1 & 1 & 1 & 4 & 0 & 0 & 1 & 1 & 5 & 4 & 1 & 1 & 1 \\
\hline 102814 & 0 & 3 & 2 & 2 & 3 & 1 & 1 & 1 & 1 & 4 & 0 & 0 & 0 & 99 & 99 & 99 & 99 & 99 & 99 \\
\hline 102815 & 1 & 5 & 2 & 1 & 3 & 2 & 1 & 1 & 1 & 4 & 0 & 0 & 0 & 99 & 99 & 99 & 99 & 99 & 99 \\
\hline 102816 & 1 & 3 & 2 & 2 & 3 & 2 & 1 & 1 & 1 & 4 & 0 & 0 & 1 & 1 & 1 & 1 & 0 & 0 & 0 \\
\hline 102817 & 0 & 4 & 2 & 2 & 3 & 1 & 1 & 1 & 1 & 4 & 0 & 0 & 0 & 99 & 99 & 99 & 99 & 99 & 99 \\
\hline 102818 & 0 & 4 & 2 & 2 & 3 & 1 & 1 & 1 & 1 & 4 & 0 & 0 & 1 & 1 & 2 & 1 & 1 & 0 & 1 \\
\hline 102819 & 1 & 3 & 2 & 2 & 3 & 2 & 1 & 1 & 1 & 4 & 0 & 0 & 1 & 1 & 3 & 6 & 1 & 1 & 1 \\
\hline 102820 & 0 & 2 & 2 & 3 & 3 & 1 & 1 & 1 & 1 & 4 & 0 & 0 & 1 & 1 & 3 & 1 & 1 & 0 & 1 \\
\hline 102821 & 1 & 2 & 2 & 3 & 3 & 2 & 3 & 1 & 1 & 1 & 0 & 0 & 1 & 1 & 2 & 1 & 1 & 0 & 1 \\
\hline 102822 & 0 & 3 & 2 & 2 & 3 & 1 & 2 & 2 & 1 & 1 & 0 & 0 & 1 & 1 & 2 & 5 & 1 & 1 & 1 \\
\hline 102823 & 0 & 3 & 2 & 2 & 3 & 1 & 3 & 1 & 1 & 1 & 0 & 0 & 1 & 1 & 1 & 1 & 0 & 0 & 0 \\
\hline 102824 & 1 & 4 & 2 & 1 & 3 & 2 & 2 & 1 & 1 & 1 & 0 & 0 & 0 & 99 & 99 & 99 & 99 & 99 & 99 \\
\hline 102825 & 1 & 4 & 2 & 2 & 3 & 2 & 2 & 1 & 1 & 1 & 0 & 0 & 1 & 1 & 1 & 1 & 0 & 0 & 0 \\
\hline 102826 & 0 & 4 & 2 & 2 & 3 & 1 & 2 & 1 & 1 & 1 & 0 & 0 & 1 & 1 & 1 & 1 & 0 & 0 & 0 \\
\hline 102827 & 0 & 5 & 2 & 2 & 3 & 1 & 3 & 1 & 1 & 1 & 0 & 0 & 1 & 1 & 1 & 1 & 0 & 0 & 0 \\
\hline 102828 & 0 & 2 & 2 & 2 & 3 & 1 & 3 & 1 & 1 & 1 & 0 & 0 & 1 & 1 & 1 & 1 & 0 & 0 & 0 \\
\hline 102829 & 1 & 5 & 2 & 1 & 3 & 2 & 3 & 1 & 1 & 1 & 0 & 0 & 1 & 1 & 1 & 1 & 0 & 0 & 0 \\
\hline 102830 & 0 & 1 & 2 & 3 & 3 & 1 & 2 & 1 & 1 & 1 & 0 & 0 & 1 & 1 & 1 & 1 & 0 & 0 & 0 \\
\hline 10291 & 1 & 5 & 2 & 2 & 3 & 2 & 2 & 1 & 1 & 2 & 0 & 0 & 1 & 1 & 1 & 6 & 0 & 1 & 1 \\
\hline 10292 & 0 & 4 & 2 & 2 & 3 & 1 & 2 & 1 & 1 & 2 & 0 & 0 & 0 & 99 & 99 & 99 & 99 & 99 & 99 \\
\hline 10293 & 0 & 5 & 2 & 1 & 3 & 1 & 2 & 1 & 1 & 2 & 0 & 0 & 0 & 99 & 99 & 99 & 99 & 99 & 99 \\
\hline 10294 & 1 & 1 & 2 & 3 & 3 & 2 & 2 & 1 & 1 & 2 & 0 & 0 & 1 & 1 & 1 & 1 & 0 & 0 & 0 \\
\hline 10295 & 1 & 2 & 2 & 3 & 3 & 2 & 2 & 1 & 1 & 2 & 0 & 0 & 1 & 1 & 1 & 5 & 0 & 1 & 1 \\
\hline 10296 & 1 & 5 & 2 & 2 & 3 & 2 & 3 & 2 & 1 & 2 & 0 & 0 & 1 & 1 & 3 & 1 & 1 & 0 & 1 \\
\hline 10297 & 1 & 3 & 2 & 2 & 3 & 2 & 2 & 1 & 1 & 2 & 0 & 0 & 0 & 99 & 99 & 99 & 99 & 99 & 99 \\
\hline 10298 & 0 & 1 & 2 & 4 & 3 & 1 & 3 & 1 & 1 & 2 & 0 & 0 & 1 & 1 & 2 & 1 & 1 & 0 & 1 \\
\hline 10299 & 1 & 4 & 2 & 2 & 3 & 2 & 2 & 1 & 1 & 2 & 0 & 0 & 1 & 1 & 3 & 5 & 1 & 1 & 1 \\
\hline 102910 & 0 & 3 & 2 & 2 & 3 & 1 & 2 & 2 & 1 & 2 & 0 & 0 & 1 & 1 & 1 & 1 & 0 & 0 & 0 \\
\hline 102911 & 1 & 3 & 2 & 3 & 3 & 2 & 2 & 1 & 1 & 2 & 0 & 0 & 1 & 1 & 3 & 1 & 1 & 0 & 1 \\
\hline 102912 & 0 & 1 & 2 & 3 & 3 & 1 & 2 & 1 & 1 & 2 & 0 & 0 & 1 & 1 & 2 & 5 & 1 & 1 & 1 \\
\hline 102913 & 1 & 3 & 2 & 2 & 3 & 2 & 2 & 1 & 1 & 2 & 0 & 0 & 1 & 1 & 3 & 1 & 1 & 0 & 1 \\
\hline 102914 & 0 & 4 & 2 & 2 & 3 & 1 & 2 & 1 & 1 & 2 & 0 & 0 & 1 & 1 & 1 & 1 & 0 & 0 & 0 \\
\hline 102915 & 0 & 4 & 2 & 1 & 3 & 1 & 2 & 1 & 1 & 2 & 0 & 0 & 1 & 1 & 2 & 1 & 1 & 0 & 1 \\
\hline 102916 & 1 & 4 & 2 & 2 & 3 & 2 & 2 & 1 & 1 & 2 & 0 & 0 & 0 & 99 & 99 & 99 & 99 & 99 & 99 \\
\hline 102917 & 1 & 3 & 2 & 2 & 3 & 2 & 2 & 1 & 1 & 2 & 0 & 0 & 1 & 1 & 1 & 5 & 0 & 1 & 1 \\
\hline 102918 & 0 & 4 & 2 & 2 & 3 & 1 & 2 & 1 & 1 & 2 & 0 & 0 & 0 & 99 & 99 & 99 & 99 & 99 & 99 \\
\hline 102919 & 0 & 4 & 2 & 2 & 3 & 1 & 2 & 1 & 1 & 2 & 0 & 0 & 0 & 99 & 99 & 99 & 99 & 99 & 99 \\
\hline 102920 & 0 & 1 & 2 & 3 & 3 & 1 & 2 & 1 & 1 & 2 & 0 & 0 & 1 & 1 & 3 & 1 & 1 & 0 & 1 \\
\hline 102921 & 0 & 1 & 2 & 2 & 3 & 1 & 2 & 1 & 1 & 2 & 0 & 0 & 1 & 1 & 2 & 1 & 1 & 0 & 1 \\
\hline 102922 & 1 & 3 & 2 & 2 & 3 & 2 & 2 & 1 & 1 & 2 & 0 & 0 & 1 & 1 & 3 & 5 & 1 & 1 & 1 \\
\hline 102923 & 1 & 3 & 2 & 2 & 3 & 2 & 2 & 1 & 1 & 2 & 0 & 0 & 1 & 1 & 1 & 1 & 0 & 0 & 0 \\
\hline 102924 & 1 & 2 & 2 & 3 & 3 & 2 & 2 & 1 & 1 & 2 & 0 & 0 & 0 & 99 & 99 & 99 & 99 & 99 & 99 \\
\hline 102925 & 0 & 2 & 2 & 4 & 3 & 1 & 3 & 1 & 1 & 2 & 0 & 0 & 1 & 1 & 2 & 1 & 1 & 0 & 1 \\
\hline 102926 & 1 & 3 & 2 & 2 & 3 & 2 & 2 & 1 & 1 & 2 & 0 & 0 & 1 & 1 & 3 & 1 & 1 & 0 & 1 \\
\hline 102927 & 1 & 3 & 2 & 2 & 3 & 2 & 2 & 1 & 1 & 2 & 0 & 0 & 0 & 99 & 99 & 99 & 99 & 99 & 99 \\
\hline 102928 & 0 & 4 & 2 & 3 & 3 & 1 & 2 & 1 & 1 & 2 & 0 & 0 & 1 & 1 & 2 & 5 & 1 & 1 & 1 \\
\hline 102929 & 0 & 4 & 2 & 2 & 3 & 1 & 2 & 1 & 1 & 2 & 0 & 0 & 1 & 1 & 2 & 6 & 1 & 1 & 1 \\
\hline
\end{tabular}




\begin{tabular}{|c|c|c|c|c|c|c|c|c|c|c|c|c|c|c|c|c|c|c|c|}
\hline 102930 & 1 & 4 & 2 & 2 & 3 & 2 & 2 & 1 & 1 & 2 & \begin{tabular}{l|l}
0 \\
\end{tabular} & 0 & 1 & 1 & 2 & 5 & 1 & 1 & 1 \\
\hline 102101 & 0 & 1 & 2 & 4 & 3 & 1 & 2 & 2 & 1 & 1 & 0 & 0 & 1 & 1 & 1 & 1 & 0 & 0 & 0 \\
\hline 102102 & 0 & 2 & 2 & 3 & 3 & 1 & 2 & 1 & 1 & 1 & 0 & 0 & 1 & 1 & 3 & 1 & 1 & 0 & 1 \\
\hline 102103 & 0 & 2 & 2 & 2 & 3 & 1 & 2 & 2 & 2 & 1 & 0 & 0 & 1 & 1 & 3 & 1 & 1 & 0 & 1 \\
\hline 102104 & 0 & 5 & 2 & 1 & 3 & 2 & 2 & 2 & 1 & 1 & 0 & 0 & 1 & 1 & 3 & 1 & 1 & 0 & 1 \\
\hline 102105 & 1 & 5 & 2 & 3 & 3 & 2 & 3 & 2 & 1 & 1 & 0 & 0 & 1 & 1 & 1 & 1 & 0 & 0 & 0 \\
\hline 102106 & 1 & 4 & 2 & 1 & 3 & 2 & 2 & 2 & 4 & 2 & 0 & 0 & 1 & 1 & 3 & 1 & 1 & 0 & 1 \\
\hline 102107 & 0 & 1 & 2 & 4 & 3 & 1 & 2 & 1 & 4 & 1 & 0 & 0 & 1 & 1 & 3 & 1 & 1 & 0 & 1 \\
\hline 102108 & 0 & 2 & 2 & 2 & 3 & 1 & 2 & 2 & 1 & 1 & 0 & 0 & 1 & 1 & 3 & 1 & 1 & 0 & 1 \\
\hline 102109 & 1 & 3 & 2 & 2 & 3 & 2 & 3 & 1 & 1 & 1 & 0 & 0 & 1 & 1 & 3 & 1 & 1 & 0 & 1 \\
\hline 1021010 & 0 & 3 & 2 & 2 & 3 & 1 & 2 & 1 & 1 & 1 & 0 & 0 & 1 & 1 & 3 & 1 & 1 & 0 & 1 \\
\hline 1021011 & 1 & 4 & 2 & 1 & 2 & 2 & 2 & 1 & 1 & 1 & 0 & 0 & 1 & 1 & 3 & 1 & 1 & 0 & 1 \\
\hline 1021012 & 0 & 1 & 2 & 2 & 3 & 1 & 3 & 1 & 1 & 1 & 0 & 0 & 1 & 1 & 3 & 1 & 1 & 0 & 1 \\
\hline 1021013 & 0 & 5 & 2 & 1 & 3 & 2 & 2 & 1 & 4 & 1 & 0 & 0 & 1 & 1 & 4 & 5 & 1 & 1 & 1 \\
\hline 1021014 & 0 & 2 & 2 & 2 & 3 & 1 & 2 & 1 & 1 & 1 & 0 & 0 & 1 & 1 & 3 & 1 & 1 & 0 & 1 \\
\hline 1021015 & 1 & 5 & 2 & 1 & 3 & 2 & 2 & 1 & 1 & 1 & 0 & 0 & 1 & 1 & 1 & 1 & 0 & 0 & 0 \\
\hline 1021016 & 1 & 4 & 2 & 2 & 3 & 2 & 2 & 2 & 4 & 1 & 0 & 0 & 1 & 1 & 3 & 1 & 1 & 0 & 1 \\
\hline 1021017 & 1 & 2 & 2 & 3 & 3 & 4 & 2 & 2 & 2 & 4 & 0 & 0 & 1 & 1 & 3 & 1 & 1 & 0 & 1 \\
\hline 1021018 & 1 & 2 & 2 & 1 & 3 & 2 & 2 & 2 & 1 & 1 & 0 & 0 & 1 & 1 & 3 & 1 & 1 & 0 & 1 \\
\hline 1021019 & 1 & 4 & 2 & 1 & 3 & 2 & 2 & 1 & 1 & 1 & 0 & 0 & 1 & 1 & 3 & 1 & 1 & 0 & 1 \\
\hline 1021020 & 1 & 5 & 2 & 2 & 2 & 2 & 2 & 1 & 1 & 1 & 0 & 0 & 1 & 0 & 5 & 5 & 1 & 1 & 1 \\
\hline 1021021 & 1 & 4 & 2 & 1 & 3 & 2 & 2 & 1 & 1 & 1 & 0 & 0 & 1 & 1 & 3 & 2 & 1 & 0 & 1 \\
\hline 1021022 & 1 & 3 & 2 & 1 & 3 & 2 & 2 & 1 & 1 & 1 & 0 & 0 & 0 & 99 & 99 & 99 & 99 & 99 & 99 \\
\hline 1021023 & 1 & 4 & 2 & 2 & 3 & 5 & 3 & 2 & 4 & 1 & 1 & 0 & 1 & 1 & 4 & 1 & 1 & 0 & 1 \\
\hline 1021024 & 1 & 3 & 2 & 1 & 3 & 2 & 2 & 1 & 1 & 1 & 0 & 0 & 1 & 1 & 3 & 1 & 1 & 0 & 1 \\
\hline 1021025 & 1 & 4 & 2 & 2 & 3 & 5 & 2 & 2 & 4 & 2 & 0 & 0 & 1 & 1 & 3 & 1 & 1 & 0 & 1 \\
\hline 1021026 & 1 & 4 & 2 & 2 & 3 & 2 & 2 & 1 & 1 & 1 & 0 & 0 & 1 & 1 & 3 & 1 & 1 & 0 & 1 \\
\hline 1021027 & 0 & 5 & 2 & 1 & 3 & 2 & 1 & 1 & 1 & 1 & 0 & 0 & 1 & 0 & 5 & 5 & 1 & 1 & 1 \\
\hline 1021028 & 0 & 5 & 2 & 1 & 3 & 2 & 2 & 2 & 4 & 2 & 0 & 0 & 1 & 1 & 1 & 1 & 0 & 0 & 0 \\
\hline 1021029 & 0 & 5 & 2 & 1 & 3 & 2 & 2 & 1 & 1 & 1 & 0 & 0 & 1 & 1 & 1 & 1 & 0 & 0 & 0 \\
\hline 1021030 & 0 & 5 & 2 & 2 & 3 & 2 & 3 & 2 & 1 & 1 & 0 & 0 & 1 & 1 & 1 & 1 & 0 & 0 & 0 \\
\hline 1021031 & 0 & 2 & 2 & 3 & 3 & 1 & 2 & 1 & 2 & 4 & 0 & 0 & 1 & 1 & 3 & 1 & 1 & 0 & 1 \\
\hline 1021032 & 0 & 2 & 2 & 4 & 3 & 1 & 3 & 2 & 4 & 1 & 0 & 0 & 1 & 1 & 3 & 1 & 1 & 0 & 1 \\
\hline 1021033 & 0 & 2 & 2 & 3 & 3 & 1 & 2 & 1 & 1 & 1 & 0 & 0 & 1 & 1 & 1 & 1 & 0 & 0 & 0 \\
\hline 102111 & 0 & 3 & 2 & 2 & 3 & 2 & 1 & 1 & 2 & 1 & 0 & 0 & 0 & 99 & 99 & 99 & 99 & 99 & 99 \\
\hline 102112 & 1 & 3 & 2 & 2 & 3 & 3 & 2 & 1 & 1 & 1 & 0 & 0 & 1 & 1 & 3 & 1 & 1 & 0 & 1 \\
\hline 102113 & 0 & 4 & 2 & 1 & 3 & 2 & 2 & 1 & 2 & 1 & 0 & 0 & 1 & 1 & 2 & 1 & 1 & 0 & 1 \\
\hline 102114 & 0 & 3 & 2 & 3 & 3 & 1 & 2 & 1 & 4 & 3 & 0 & 0 & 1 & 1 & 4 & 1 & 1 & 0 & 1 \\
\hline 102115 & 1 & 2 & 2 & 2 & 3 & 4 & 2 & 1 & 4 & 3 & 0 & 0 & 1 & 1 & 1 & 1 & 0 & 0 & 0 \\
\hline 102116 & 0 & 2 & 2 & 2 & 2 & 1 & 2 & 2 & 4 & 3 & 0 & 0 & 1 & 1 & 3 & 1 & 1 & 0 & 1 \\
\hline 102117 & 1 & 2 & 2 & 5 & 3 & 5 & 3 & 1 & 4 & 1 & 1 & 0 & 1 & 1 & 1 & 1 & 0 & 0 & 0 \\
\hline 102118 & 1 & 1 & 2 & 3 & 3 & 5 & 2 & 2 & 4 & 4 & 0 & 0 & 1 & 1 & 2 & 5 & 1 & 1 & 1 \\
\hline 102119 & 1 & 5 & 2 & 4 & 3 & 4 & 2 & 1 & 2 & 1 & 0 & 0 & 1 & 1 & 1 & 1 & 0 & 0 & 0 \\
\hline 1021110 & 0 & 4 & 2 & 1 & 3 & 2 & 2 & 1 & 4 & 2 & 0 & 0 & 0 & 99 & 99 & 99 & 99 & 99 & 99 \\
\hline 1021111 & 1 & 2 & 2 & 4 & 3 & 4 & 2 & 1 & 4 & 2 & 0 & 0 & 1 & 0 & 5 & 5 & 1 & 1 & 1 \\
\hline 1021112 & 1 & 2 & 2 & 3 & 3 & 2 & 2 & 1 & 4 & 2 & 0 & 0 & 1 & 1 & 1 & 1 & 0 & 0 & 0 \\
\hline 1021113 & 1 & 1 & 2 & 1 & 2 & 4 & 2 & 1 & 2 & 1 & 0 & 0 & 1 & 0 & 5 & 5 & 1 & 1 & 1 \\
\hline 1021114 & 0 & 3 & 2 & 1 & 3 & 2 & 1 & 1 & 2 & 1 & 0 & 0 & 1 & 0 & 5 & 5 & 1 & 1 & 1 \\
\hline 1021115 & 0 & 3 & 2 & 2 & 3 & 1 & 2 & 1 & 2 & 1 & 0 & 0 & 1 & 1 & 3 & 1 & 1 & 0 & 1 \\
\hline 1021116 & 0 & 3 & 2 & 2 & 3 & 1 & 2 & 2 & 1 & 1 & 0 & 0 & 1 & 0 & 5 & 5 & 1 & 1 & 1 \\
\hline 1021117 & 1 & 5 & 2 & 2 & 2 & 5 & 2 & 1 & 4 & 3 & 1 & 1 & 1 & 1 & 1 & 1 & 0 & 0 & 0 \\
\hline 1021118 & 0 & 4 & 2 & 2 & 3 & 1 & 2 & 1 & 4 & 3 & 0 & 0 & 1 & 1 & 1 & 1 & 0 & 0 & 0 \\
\hline 1021119 & 0 & 4 & 2 & 2 & 3 & 1 & 2 & 2 & 4 & 3 & 0 & 0 & 1 & 1 & 1 & 1 & 0 & 0 & 0 \\
\hline 1021120 & 1 & 3 & 2 & 1 & 3 & 2 & 2 & 2 & 4 & 2 & 0 & 0 & 1 & 1 & 4 & 1 & 1 & 0 & 1 \\
\hline 1021121 & 1 & 5 & 2 & 1 & 3 & 2 & 1 & 1 & 4 & 1 & 0 & 0 & 0 & 99 & 99 & 99 & 99 & 99 & 99 \\
\hline 1021122 & 1 & 5 & 2 & 1 & 3 & 2 & 3 & 2 & 4 & 2 & 0 & 0 & 1 & 1 & 3 & 1 & 1 & 0 & 1 \\
\hline
\end{tabular}




\begin{tabular}{|c|c|c|c|c|c|c|c|c|c|c|c|c|c|c|c|c|c|c|c|}
\hline 1021123 & 0 & 5 & 2 & 2 & 3 & 2 & 1 & 1 & 4 & 3 & 0 & 0 & 1 & 0 & 5 & 5 & 1 & 1 & 1 \\
\hline 1021124 & 1 & 4 & 2 & 1 & 3 & 4 & 2 & 2 & 4 & 4 & 0 & 0 & 1 & 1 & 2 & 1 & 1 & 0 & 1 \\
\hline 1021125 & 1 & 2 & 2 & 3 & 3 & 4 & 3 & 1 & 4 & 2 & 0 & 0 & 1 & 1 & 3 & 1 & 1 & 0 & 1 \\
\hline 1021126 & 0 & 4 & 2 & 2 & 3 & 5 & 3 & 2 & 4 & 3 & 0 & 0 & 1 & 1 & 3 & 1 & 1 & 0 & 1 \\
\hline 1021127 & 1 & 3 & 2 & 1 & 3 & 4 & 2 & 1 & 4 & 2 & 0 & 0 & 1 & 1 & 1 & 1 & \begin{tabular}{l|l}
0 \\
\end{tabular} & 0 & 0 \\
\hline 1021128 & 1 & 3 & 2 & 4 & 3 & 5 & 3 & 2 & 4 & 1 & 0 & 0 & 1 & 1 & 1 & 1 & 0 & 0 & 0 \\
\hline 1021129 & 1 & 3 & 2 & 4 & 3 & 5 & 3 & 2 & 4 & 1 & 0 & 0 & 1 & 1 & 3 & 1 & 1 & 0 & 1 \\
\hline 1021130 & 1 & 3 & 2 & 4 & 3 & 5 & 3 & 1 & 4 & 3 & 0 & 0 & 1 & 1 & 1 & 1 & 0 & 0 & 0 \\
\hline 1021131 & 0 & 2 & 2 & 4 & 3 & 5 & 2 & 1 & 4 & 3 & 0 & 0 & 1 & 1 & 1 & 1 & 0 & 0 & 0 \\
\hline 1021132 & 0 & 3 & 2 & 4 & 3 & 1 & 3 & 2 & 4 & 2 & 0 & 0 & 1 & 1 & 3 & 1 & 1 & 0 & 1 \\
\hline 1021133 & 0 & 4 & 2 & 2 & 3 & 5 & 3 & 1 & 4 & 3 & 0 & \begin{tabular}{l|l}
0 \\
\end{tabular} & 1 & 1 & 1 & 1 & 0 & 0 & 0 \\
\hline 1021134 & 1 & 4 & 2 & 4 & 3 & 5 & 3 & 2 & 4 & 2 & 0 & 0 & 1 & 1 & 1 & 1 & 0 & 0 & 0 \\
\hline 1021135 & 1 & 2 & 2 & 5 & 2 & 5 & 3 & 1 & 4 & 2 & 0 & 0 & 1 & 1 & 1 & 1 & 0 & 0 & 0 \\
\hline 1021136 & 0 & 2 & 2 & 4 & 3 & 1 & 2 & 2 & 4 & 4 & 0 & 0 & 1 & 1 & 1 & 1 & 0 & 0 & 0 \\
\hline 1021137 & 0 & 5 & 2 & 2 & 3 & 2 & 2 & 2 & 4 & 3 & 0 & 0 & 1 & 1 & 1 & 1 & 0 & 0 & 0 \\
\hline 1021138 & 1 & 2 & 2 & 5 & 2 & 5 & 3 & 1 & 4 & 2 & 0 & 0 & 1 & 1 & 1 & 1 & 0 & 0 & 0 \\
\hline 1021139 & 0 & 4 & 2 & 2 & 3 & 1 & 2 & 1 & 4 & 2 & 0 & 0 & 1 & 1 & 1 & 1 & 0 & 0 & $\overline{0}$ \\
\hline 102121 & 0 & 5 & 2 & 1 & 3 & 2 & 2 & 1 & 2 & 1 & 0 & 0 & 0 & 99 & 99 & 99 & 99 & 99 & 99 \\
\hline \begin{tabular}{l|}
102122 \\
\end{tabular} & 0 & 4 & 2 & 2 & 3 & 2 & 3 & 1 & 1 & 1 & 0 & 0 & 1 & 1 & 1 & 1 & 0 & 0 & 0 \\
\hline 102123 & 1 & 5 & 2 & 5 & 2 & 5 & 3 & 1 & 2 & 1 & 0 & 0 & 1 & 1 & 1 & 1 & \begin{tabular}{l|l}
0 \\
\end{tabular} & 0 & 0 \\
\hline \begin{tabular}{|l|}
102124 \\
\end{tabular} & 1 & 4 & 2 & 1 & 3 & 2 & 2 & 1 & 1 & 1 & 0 & 0 & 0 & 99 & 99 & 99 & 99 & 99 & 99 \\
\hline \begin{tabular}{l|}
102125 \\
\end{tabular} & 0 & 3 & 2 & 3 & 3 & 1 & 2 & 2 & 4 & 1 & 0 & 0 & 1 & 1 & 3 & 1 & 1 & 0 & 1 \\
\hline 102126 & 1 & 3 & 2 & 2 & 3 & 2 & 2 & 1 & 2 & 1 & 0 & 0 & 1 & 1 & 3 & 6 & 1 & 1 & 1 \\
\hline \begin{tabular}{|l|}
102127 \\
\end{tabular} & 0 & 3 & 2 & 1 & 3 & 1 & 2 & 2 & 1 & 1 & 0 & 0 & 1 & 1 & 3 & 1 & 1 & 0 & 1 \\
\hline 102128 & 1 & 3 & 2 & 3 & 3 & 2 & 3 & 1 & 4 & 1 & 0 & 0 & 1 & 1 & 3 & 1 & 1 & 0 & 1 \\
\hline \begin{tabular}{l|}
102129 \\
\end{tabular} & 0 & 1 & 2 & 4 & 3 & 1 & 3 & 1 & 4 & 1 & 0 & 0 & 1 & 1 & 3 & 1 & 1 & 0 & 1 \\
\hline 1021210 & 1 & 3 & 2 & 2 & 3 & 2 & 3 & 2 & 2 & 1 & 0 & 0 & 1 & 1 & 1 & 1 & 0 & 0 & $\overline{0}$ \\
\hline 1021211 & 0 & 1 & 2 & 5 & 3 & 5 & 3 & 1 & 2 & 3 & 0 & 0 & 1 & 1 & 1 & 1 & 0 & 0 & $\overline{0}$ \\
\hline 1021212 & 1 & 2 & 2 & 2 & 3 & 2 & 3 & 2 & 2 & 3 & 0 & 0 & 1 & 1 & 2 & 1 & 1 & 0 & 1 \\
\hline 1021213 & 0 & 5 & 2 & 2 & 3 & 1 & 2 & 2 & 2 & 2 & 0 & 0 & 1 & 1 & 1 & 1 & \begin{tabular}{l|l}
0 \\
\end{tabular} & 0 & 0 \\
\hline 1021214 & 0 & 4 & 2 & 2 & 3 & 1 & 2 & 2 & 2 & 2 & 0 & 0 & 1 & 1 & 4 & 1 & 1 & 0 & 1 \\
\hline 1021215 & 0 & 4 & 2 & 2 & 3 & 1 & 2 & 2 & 2 & 2 & 0 & 0 & 1 & 1 & 4 & 1 & 1 & 0 & 1 \\
\hline 1021216 & 1 & 5 & 2 & 1 & 3 & 2 & 2 & 2 & 2 & 2 & 0 & 0 & 1 & 1 & 3 & 5 & 1 & 1 & 1 \\
\hline 1021217 & 0 & 4 & 2 & 2 & 3 & 1 & 2 & 1 & 2 & 1 & 0 & 0 & 1 & 1 & 4 & 1 & 1 & 0 & 1 \\
\hline 1021218 & 1 & 1 & 2 & 3 & 3 & 4 & 3 & 1 & 2 & 1 & 0 & 0 & 1 & 1 & 3 & 5 & 1 & 1 & 1 \\
\hline 1021219 & 1 & 3 & 2 & 3 & 3 & 2 & 2 & 2 & 2 & 1 & 0 & 0 & 1 & 1 & 1 & 5 & 0 & 1 & 1 \\
\hline 1021220 & 1 & 4 & 2 & 2 & 3 & 2 & 2 & 1 & 2 & 1 & 0 & 0 & 1 & 0 & 5 & 5 & 1 & 1 & 1 \\
\hline 1021221 & 1 & 5 & 2 & 2 & 3 & 2 & 3 & 1 & 1 & 1 & 0 & 0 & 1 & 1 & 1 & 1 & 0 & 0 & 0 \\
\hline 1021222 & 0 & 4 & 2 & 1 & 3 & 1 & 2 & 2 & 2 & 2 & 0 & 0 & 1 & 1 & 1 & 1 & 0 & 0 & $\overline{0}$ \\
\hline 1021223 & 1 & 2 & 2 & 5 & 3 & 5 & 3 & 1 & 4 & 1 & 0 & 0 & 1 & 1 & 2 & 1 & 1 & 0 & 1 \\
\hline 1021224 & 0 & 4 & 2 & 5 & 3 & 5 & 3 & 2 & 4 & 1 & 1 & 1 & 1 & 1 & 2 & 1 & 1 & 0 & 1 \\
\hline 1021225 & 0 & 5 & 2 & 2 & 3 & 1 & 2 & 2 & 2 & 1 & 0 & 0 & 1 & 1 & 2 & 1 & 1 & 0 & 1 \\
\hline 1021226 & 0 & 4 & 2 & 1 & 3 & 1 & 2 & 1 & 2 & 2 & 0 & 0 & 1 & 0 & 5 & 5 & 1 & 1 & 1 \\
\hline 1021227 & 1 & 2 & 2 & 1 & 3 & 4 & 2 & 1 & 2 & 2 & 0 & 0 & 1 & 1 & 1 & 1 & 0 & 0 & 0 \\
\hline 1021228 & 1 & 5 & 2 & 1 & 3 & 2 & 2 & 2 & 2 & 1 & 0 & 0 & 1 & 0 & 5 & 5 & 1 & 1 & 1 \\
\hline 1021229 & 0 & 2 & 2 & 2 & 4 & 1 & 2 & 2 & 4 & 1 & 0 & 0 & 1 & 1 & 3 & 1 & 1 & 0 & 1 \\
\hline 1021230 & 1 & 2 & 2 & 2 & 3 & 2 & 2 & 2 & 2 & 1 & 0 & 0 & 1 & 1 & 4 & 1 & 1 & 0 & 1 \\
\hline 1021231 & 1 & 3 & 2 & 1 & 3 & 4 & 2 & 2 & 2 & 1 & 0 & 0 & 1 & 1 & 3 & 1 & 1 & 0 & 1 \\
\hline 1021232 & 1 & 4 & 2 & 1 & 2 & 4 & 2 & 1 & 2 & 1 & 0 & 0 & 1 & 0 & 5 & 5 & 1 & 1 & 1 \\
\hline 1021233 & 1 & 1 & 2 & 5 & 3 & 3 & 3 & 1 & 2 & 1 & 1 & 1 & 1 & 1 & 2 & 1 & 1 & 0 & 1 \\
\hline 1021234 & 0 & 5 & 2 & 1 & 2 & 1 & 2 & 2 & 4 & 2 & 0 & 0 & 1 & 1 & 1 & 1 & 0 & 0 & 0 \\
\hline 1021235 & 0 & 2 & 2 & 5 & 3 & 5 & 2 & 2 & 4 & 2 & 0 & 0 & 1 & 1 & 1 & 1 & 0 & 0 & 0 \\
\hline 103141 & 1 & 4 & 2 & 3 & 3 & 2 & 2 & 1 & 2 & 1 & 1 & 1 & 0 & 99 & 99 & 99 & 99 & 99 & 99 \\
\hline \begin{tabular}{l|l|}
103142 \\
\end{tabular} & 1 & 2 & 2 & 3 & 3 & 2 & 2 & 2 & 2 & 1 & 0 & 0 & 0 & 99 & 99 & 99 & 99 & 99 & 99 \\
\hline \begin{tabular}{|l|}
103143 \\
\end{tabular} & 1 & 5 & 2 & 3 & 3 & 2 & 2 & 2 & 2 & 1 & 1 & 0 & 0 & 99 & 99 & 99 & 99 & 99 & $\overline{99}$ \\
\hline 103144 & 1 & 3 & 2 & 3 & 3 & 2 & 2 & 2 & 2 & 1 & 0 & 0 & 1 & 1 & 1 & 1 & \begin{tabular}{l|}
0 \\
\end{tabular} & 0 & \\
\hline
\end{tabular}




\begin{tabular}{|c|c|c|c|c|c|c|c|c|c|c|c|c|c|c|c|c|c|c|c|}
\hline 103145 & 1 & 3 & 2 & 3 & 3 & 2 & 2 & 1 & 2 & 1 & 0 & 0 & 0 & 99 & 99 & 99 & \begin{tabular}{l|l}
99 \\
\end{tabular} & 99 & 99 \\
\hline 103146 & 1 & 5 & 2 & 1 & 3 & 2 & 2 & 1 & 2 & 1 & 0 & 0 & 0 & 99 & 99 & 99 & $\begin{array}{ll}99 \\
\end{array}$ & 99 & 99 \\
\hline 103147 & 1 & 5 & 2 & 1 & 3 & 2 & 1 & 2 & 2 & 1 & 0 & 0 & 0 & 99 & 99 & 99 & 99 & 99 & 99 \\
\hline 103148 & 1 & 4 & 2 & 3 & 3 & 2 & 2 & 2 & 2 & 1 & 0 & 0 & 0 & 99 & 99 & 99 & 99 & 99 & 99 \\
\hline 103149 & 1 & 3 & 2 & 2 & 3 & 2 & 2 & 2 & 2 & 1 & 1 & 0 & 0 & 99 & 99 & 99 & 999 & 99 & 99 \\
\hline 1031410 & 1 & 4 & 2 & 4 & 3 & 2 & 2 & 1 & 2 & 1 & 0 & 0 & 1 & 1 & 1 & 1 & \begin{tabular}{l|l}
0 \\
\end{tabular} & 0 & $\overline{0}$ \\
\hline 1031411 & 0 & 5 & 2 & 2 & 3 & 1 & 2 & 1 & 3 & 1 & 1 & 1 & 0 & 99 & 99 & 99 & $\begin{array}{ll}99 \\
\end{array}$ & 99 & 99 \\
\hline 1031412 & 0 & 3 & 2 & 2 & 3 & 1 & 1 & 1 & 3 & 1 & 0 & 0 & 0 & 99 & 99 & 99 & 99 & 99 & 99 \\
\hline 1031413 & 0 & 4 & 2 & 1 & 3 & 1 & 2 & 1 & 3 & 1 & 0 & 0 & 0 & 99 & 99 & 99 & 99 & 99 & 99 \\
\hline 1031414 & 0 & 3 & 2 & 2 & 3 & 1 & 2 & 1 & 3 & 1 & 0 & 0 & 0 & 99 & 99 & 99 & 99 & 99 & 99 \\
\hline 1031415 & 0 & 3 & 2 & 1 & 3 & 1 & 2 & 1 & 3 & 1 & 0 & 0 & 1 & 1 & 1 & 1 & 0 & 0 & 0 \\
\hline 1031416 & 0 & 3 & 2 & 2 & 3 & 1 & 2 & 1 & 3 & 1 & 0 & 0 & 0 & 99 & 99 & 99 & 99 & 99 & 99 \\
\hline 1031417 & 0 & 5 & 2 & 2 & 3 & 1 & 1 & 1 & 3 & 1 & 0 & 0 & 0 & 99 & 99 & 99 & 99 & 99 & 99 \\
\hline 1031418 & 0 & 5 & 2 & 2 & 3 & 1 & 2 & 2 & 3 & 1 & 0 & 0 & 0 & 99 & 99 & 99 & 99 & 99 & 99 \\
\hline 1031419 & 0 & 4 & 2 & 2 & 3 & 1 & 1 & 1 & 3 & 1 & 0 & 0 & 0 & 99 & 99 & 99 & 99 & 99 & 99 \\
\hline 1031420 & 0 & 5 & 2 & 2 & 3 & 1 & 2 & 2 & 3 & 1 & 0 & 0 & 0 & 99 & 99 & 99 & 99 & 99 & 99 \\
\hline 1031421 & 0 & 4 & 2 & 2 & 3 & 1 & 1 & 1 & 3 & 1 & 0 & 0 & 0 & 99 & 99 & 99 & 999 & 99 & 99 \\
\hline 1031422 & 0 & 4 & 2 & 2 & 3 & 1 & 1 & 1 & 3 & 1 & 0 & 0 & 0 & 99 & 99 & 99 & 99 & 99 & 99 \\
\hline 1031423 & 0 & 1 & 2 & 2 & 3 & 1 & 1 & 1 & 3 & 1 & 0 & 0 & 1 & 1 & 1 & 1 & 0 & 0 & 0 \\
\hline 1031424 & 0 & 4 & 2 & 1 & 3 & 1 & 2 & 2 & 3 & 1 & 0 & 0 & 0 & 99 & 99 & 99 & 99 & 99 & 99 \\
\hline 1031425 & 1 & 4 & 2 & 1 & 3 & 2 & 1 & 1 & 3 & 1 & 0 & 0 & 0 & 99 & 99 & 99 & 99 & 99 & 99 \\
\hline 1031426 & 0 & 2 & 2 & 2 & 3 & 1 & 1 & 1 & 3 & 1 & 0 & 0 & 0 & 99 & 99 & 99 & 99 & 99 & 99 \\
\hline 1031427 & 0 & 3 & 2 & 2 & 3 & 1 & 1 & 1 & 3 & 1 & 0 & 0 & 1 & 1 & 1 & 1 & \begin{tabular}{l|l}
0 \\
\end{tabular} & 0 & 0 \\
\hline 1031428 & 0 & 1 & 2 & 4 & 3 & 1 & 1 & 1 & 3 & 1 & 0 & 0 & 0 & 99 & 99 & 99 & 99 & 99 & 99 \\
\hline 1031429 & 0 & 4 & 2 & 3 & 3 & 1 & 2 & 1 & 3 & 1 & 0 & 0 & 1 & 1 & 3 & 1 & 1 & 0 & 1 \\
\hline 1031430 & 0 & 3 & 2 & 2 & 3 & 1 & 1 & 1 & 3 & 1 & 0 & 0 & 0 & 99 & 99 & 99 & 99 & 99 & 99 \\
\hline 103131 & 0 & 3 & 2 & 2 & 3 & 1 & 2 & 1 & 2 & 1 & 0 & 0 & 1 & 1 & 1 & 1 & 0 & 0 & 0 \\
\hline 103132 & 0 & 2 & 2 & 5 & 3 & 5 & 2 & 2 & 2 & 4 & 0 & 0 & 1 & 1 & 1 & 1 & 0 & 0 & 0 \\
\hline 103133 & 0 & 2 & 2 & 2 & 3 & 1 & 2 & 2 & 2 & 1 & 0 & 0 & 1 & 1 & 4 & 1 & 1 & 0 & 1 \\
\hline 103134 & 0 & 2 & 2 & 3 & 3 & 1 & 3 & 2 & 2 & 1 & 0 & 0 & 1 & 1 & 1 & 1 & 0 & 0 & 0 \\
\hline 103135 & 0 & 2 & 2 & 5 & 3 & 5 & 3 & 2 & 2 & 1 & 0 & 0 & 1 & 1 & 1 & 1 & 0 & 0 & 0 \\
\hline 103136 & 1 & 4 & 2 & 2 & 3 & 2 & 1 & 1 & 2 & 1 & 0 & 0 & 1 & 1 & 4 & 1 & 1 & 0 & 1 \\
\hline 103137 & 0 & 3 & 2 & 2 & 3 & 1 & 1 & 2 & 2 & 1 & 0 & 0 & 1 & 1 & 4 & 1 & 1 & 0 & 1 \\
\hline 103138 & 0 & 2 & 2 & 2 & 3 & 1 & 1 & 2 & 2 & 1 & 0 & 0 & 0 & 99 & 99 & 99 & 99 & 99 & 99 \\
\hline 103139 & 0 & 3 & 2 & 4 & 3 & 1 & 1 & 1 & 2 & 1 & 0 & 0 & 1 & 1 & 1 & 1 & 0 & 0 & 0 \\
\hline 1031310 & 1 & 5 & 2 & 2 & 3 & 2 & 1 & 1 & 2 & 1 & 0 & 0 & 1 & 1 & 3 & 1 & 1 & 0 & 1 \\
\hline 1031311 & 0 & 4 & 2 & 2 & 3 & 1 & 2 & 1 & 2 & 1 & 0 & 0 & 1 & 1 & 3 & 1 & 1 & 0 & 1 \\
\hline 1031312 & 1 & 4 & 2 & 2 & 3 & 5 & 1 & 1 & 2 & 1 & 0 & 0 & 0 & 99 & 99 & 99 & 99 & 99 & 99 \\
\hline 1031313 & 1 & 3 & 2 & 4 & 3 & 2 & 2 & 2 & 2 & 1 & 0 & 0 & 1 & 1 & 4 & 1 & 1 & 0 & 1 \\
\hline 1031314 & 1 & 2 & 2 & 4 & 3 & 2 & 2 & 2 & 2 & 1 & 0 & 0 & 1 & 1 & 4 & 1 & 1 & 0 & 1 \\
\hline 1031315 & 0 & 4 & 2 & 2 & 3 & 1 & 1 & 1 & 2 & 1 & 0 & 0 & 1 & 1 & 3 & 1 & 1 & 0 & 1 \\
\hline 1031316 & 0 & 4 & 2 & 2 & 3 & 1 & 1 & 1 & 2 & 1 & 0 & 0 & 1 & 1 & 4 & 1 & 1 & 0 & 1 \\
\hline 1031317 & 1 & 2 & 2 & 2 & 3 & 2 & 2 & 2 & 2 & 1 & 0 & 0 & 0 & 99 & 99 & 99 & 99 & 99 & 99 \\
\hline 1031318 & 0 & 3 & 2 & 2 & 3 & 2 & 1 & 1 & 2 & 1 & 0 & 0 & 1 & 1 & 4 & 1 & 1 & 0 & 1 \\
\hline 1031319 & 0 & 1 & 2 & 4 & 3 & 5 & 3 & 1 & 2 & 1 & 0 & 0 & 1 & 1 & 4 & 1 & 1 & 0 & 1 \\
\hline 1031320 & 0 & 1 & 2 & 4 & 3 & 1 & 2 & 1 & 2 & 1 & 0 & 0 & 1 & 1 & 1 & 1 & 0 & 0 & 0 \\
\hline 1031321 & 0 & 5 & 2 & 2 & 3 & 1 & 1 & 1 & 2 & 1 & 0 & 0 & 0 & 99 & 99 & 99 & 99 & 99 & 99 \\
\hline 1031322 & 0 & 5 & 2 & 2 & 3 & 1 & 2 & 1 & 2 & 1 & 0 & 0 & 0 & 99 & 99 & 99 & 99 & 99 & 99 \\
\hline 1031323 & 0 & 2 & 2 & 2 & 3 & 1 & 2 & 2 & 2 & 1 & 0 & 0 & 0 & 99 & 99 & 99 & 999 & 99 & 99 \\
\hline 1031324 & 0 & 3 & 2 & 2 & 3 & 1 & 2 & 1 & 2 & 1 & 0 & 0 & 1 & 1 & 4 & 1 & 1 & 0 & 1 \\
\hline 1031325 & 0 & 4 & 2 & 2 & 3 & 1 & 2 & 1 & 2 & 1 & 0 & 0 & 1 & 1 & 3 & 1 & 1 & 0 & 1 \\
\hline 1031326 & 0 & 2 & 2 & 2 & 3 & 1 & 2 & 2 & 2 & 1 & 0 & 0 & 1 & 1 & 1 & 1 & 0 & 0 & 0 \\
\hline 1031327 & 0 & 3 & 2 & 2 & 3 & 1 & 2 & 2 & 2 & 1 & 0 & 0 & 1 & 1 & 4 & 1 & 1 & 0 & 1 \\
\hline 1031328 & 0 & 5 & 2 & 2 & 3 & 1 & 1 & 1 & 2 & 1 & 0 & 0 & 0 & 99 & 99 & 99 & 99 & 99 & 99 \\
\hline 1031329 & 0 & 3 & 2 & 2 & 3 & 1 & 1 & 2 & 2 & 1 & 0 & 0 & 1 & 1 & 3 & 1 & 1 & 0 & 1 \\
\hline 1031330 & 0 & 3 & 2 & 2 & 3 & 1 & 2 & 1 & 2 & 1 & 0 & 0 & 0 & 99 & 99 & 99 & (99 & 99 & 99 \\
\hline
\end{tabular}




\begin{tabular}{|c|c|c|c|c|c|c|c|c|c|c|c|c|c|c|c|c|c|c|c|}
\hline 103151 & 0 & 4 & 2 & 1 & 3 & 1 & 1 & 1 & 2 & 1 & 0 & 0 & 0 & 99 & 99 & 99 & 99 & 99 & 99 \\
\hline 103152 & 0 & 4 & 2 & 2 & 3 & 1 & 2 & 1 & 2 & 1 & 0 & 0 & 1 & 1 & 3 & 1 & 1 & 0 & 1 \\
\hline 103153 & 0 & 4 & 2 & 2 & 3 & 1 & 2 & 2 & 2 & 1 & 0 & 0 & 0 & 99 & 99 & 99 & 99 & 99 & 99 \\
\hline 103154 & 0 & 1 & 2 & 2 & 3 & 1 & 2 & 2 & 2 & 1 & 0 & 0 & 0 & 99 & 99 & 99 & 99 & 99 & 99 \\
\hline 103155 & 0 & 2 & 2 & 2 & 3 & 1 & 2 & 1 & 2 & 1 & 0 & 0 & 1 & 1 & 3 & 1 & 1 & 0 & 1 \\
\hline 103156 & 1 & 4 & 2 & 2 & 3 & 2 & 2 & 1 & 2 & 1 & 0 & 0 & 0 & 99 & 99 & 99 & 99 & 99 & 99 \\
\hline 103157 & 0 & 1 & 2 & 4 & 3 & 1 & 1 & 1 & 2 & 1 & 0 & 0 & 0 & 99 & 99 & 99 & 99 & 99 & 99 \\
\hline 103158 & 0 & 4 & 2 & 2 & 3 & 1 & 1 & 1 & 2 & 1 & 0 & 0 & 0 & 99 & 99 & 99 & 99 & 99 & 99 \\
\hline 103159 & 0 & 5 & 2 & 2 & 3 & 1 & 2 & 1 & 2 & 1 & 1 & 1 & 0 & 99 & 99 & 99 & 99 & 99 & 99 \\
\hline 1031510 & 0 & 3 & 2 & 2 & 3 & 1 & 2 & 2 & 2 & 1 & 0 & 0 & 0 & 99 & 99 & 99 & 99 & 99 & 99 \\
\hline 1031511 & 0 & 2 & 2 & 3 & 3 & 1 & 2 & 2 & 2 & 1 & 0 & 0 & 0 & 99 & 99 & 99 & 99 & 99 & 99 \\
\hline 1031512 & 0 & 2 & 2 & 2 & 3 & 1 & 1 & 2 & 2 & 1 & 0 & 0 & 0 & 99 & 99 & 99 & 99 & 99 & 99 \\
\hline 1031513 & 0 & 1 & 2 & 4 & 3 & 1 & 1 & 2 & 2 & 1 & 0 & 0 & 0 & 99 & 99 & 99 & 99 & 99 & 99 \\
\hline 1031514 & 0 & 1 & 2 & 3 & 3 & 1 & 1 & 1 & 2 & 1 & 0 & 0 & 0 & 99 & 99 & 99 & 99 & 99 & 99 \\
\hline 1031515 & 0 & 1 & 2 & 4 & 3 & 1 & 1 & 1 & 2 & 1 & 0 & 0 & 0 & 99 & 99 & 99 & 99 & 99 & 99 \\
\hline 1031516 & 0 & 2 & 2 & 3 & 3 & 1 & 1 & 2 & 2 & 1 & 0 & 0 & 1 & 1 & 2 & 1 & 1 & 0 & 1 \\
\hline 1031517 & 0 & 4 & 2 & 2 & 3 & 1 & 1 & 1 & 2 & 1 & 0 & 0 & 0 & 99 & 99 & 99 & 99 & 99 & 99 \\
\hline 1031518 & 0 & 4 & 2 & 3 & 3 & 1 & 2 & 2 & 2 & 1 & 0 & 0 & 1 & 1 & 1 & 1 & 0 & 0 & 0 \\
\hline 1031519 & 0 & 1 & 2 & 4 & 3 & 1 & 1 & 1 & 2 & 1 & 0 & 0 & 1 & 1 & 1 & 1 & 0 & 0 & 0 \\
\hline 1031520 & 1 & 4 & 2 & 2 & 3 & 2 & 2 & 1 & 2 & 1 & 0 & 0 & 0 & 99 & 99 & 99 & 99 & 99 & 99 \\
\hline 1031521 & 0 & 4 & 2 & 1 & 3 & 1 & 1 & 1 & 2 & 1 & 0 & 0 & 0 & 99 & 99 & 99 & 99 & 99 & 99 \\
\hline 1031522 & 1 & 5 & 2 & 2 & 3 & 2 & 2 & 2 & 2 & 1 & 0 & 0 & 0 & 99 & 99 & 99 & 99 & 99 & 99 \\
\hline 1031523 & 1 & 5 & 2 & 2 & 3 & 2 & 3 & 2 & 2 & 1 & 1 & 0 & 1 & 1 & 1 & 1 & 0 & 0 & 0 \\
\hline 1031524 & 0 & 5 & 2 & 1 & 3 & 1 & 2 & 1 & 2 & 1 & 0 & 0 & 0 & 99 & 99 & 99 & 99 & 99 & 99 \\
\hline 1031525 & 1 & 4 & 2 & 1 & 3 & 2 & 2 & 1 & 2 & 1 & 0 & 0 & 0 & 99 & 99 & 99 & 99 & 99 & 99 \\
\hline 1031526 & 0 & 5 & 2 & 1 & 3 & 1 & 1 & 1 & 2 & 1 & 0 & 0 & 0 & 99 & 99 & 99 & 99 & 99 & 99 \\
\hline 1031527 & 0 & 4 & 2 & 2 & 3 & 1 & 2 & 1 & 2 & 1 & 0 & 0 & 0 & 99 & 99 & 99 & 99 & 99 & 99 \\
\hline 1031528 & 0 & 1 & 2 & 3 & 3 & 1 & 2 & 2 & 2 & 1 & 0 & 0 & 1 & 1 & 1 & 1 & 0 & 0 & 0 \\
\hline 1031529 & 0 & 3 & 2 & 2 & 3 & 1 & 2 & 1 & 2 & 1 & 0 & 0 & 1 & 1 & 2 & 1 & 1 & 0 & 1 \\
\hline 1031530 & 0 & 2 & 2 & 5 & 3 & 5 & 3 & 1 & 2 & 1 & 0 & 0 & 1 & 1 & 1 & 1 & 0 & 0 & 0 \\
\hline 103161 & 0 & 3 & 2 & 2 & 3 & 1 & 2 & 2 & 1 & 1 & 0 & 0 & 0 & 99 & 99 & 99 & 99 & 99 & 99 \\
\hline 103162 & 0 & 3 & 2 & 3 & 3 & 1 & 2 & 2 & 1 & 1 & 0 & 0 & 0 & 99 & 99 & 99 & 99 & 99 & 99 \\
\hline 103163 & 0 & 2 & 2 & 3 & 3 & 1 & 3 & 2 & 1 & 1 & 0 & 0 & 1 & 1 & 1 & 1 & 0 & 0 & 0 \\
\hline 103164 & 0 & 2 & 2 & 3 & 3 & 1 & 2 & 1 & 1 & 1 & 0 & 0 & 1 & 1 & 1 & 1 & 0 & 0 & 0 \\
\hline 103165 & 0 & 4 & 2 & 4 & 3 & 1 & 1 & 1 & 1 & 1 & 0 & 0 & 1 & 1 & 1 & 1 & 0 & 0 & 0 \\
\hline 103166 & 0 & 4 & 2 & 2 & 3 & 1 & 1 & 1 & 1 & 1 & 0 & 0 & 1 & 1 & 1 & 1 & 0 & 0 & 0 \\
\hline 103167 & 0 & 2 & 2 & 2 & 3 & 1 & 1 & 2 & 1 & 1 & 0 & 0 & 0 & 99 & 99 & 99 & 99 & 99 & 99 \\
\hline 103168 & 0 & 5 & 2 & 2 & 3 & 1 & 2 & 1 & 1 & 1 & 0 & 0 & 1 & 1 & 1 & 1 & 0 & 0 & 0 \\
\hline 103169 & 0 & 5 & 2 & 2 & 3 & 1 & 1 & 2 & 1 & 1 & 0 & 0 & 0 & 99 & 99 & 99 & 99 & 99 & 99 \\
\hline 1031610 & 0 & 1 & 2 & 4 & 3 & 1 & 2 & 1 & 1 & 1 & 0 & 0 & 0 & 99 & 99 & 99 & 99 & 99 & 99 \\
\hline 1031611 & 0 & 1 & 2 & 2 & 3 & 1 & 1 & 2 & 1 & 1 & 0 & 0 & 1 & 1 & 4 & 1 & 1 & 0 & 1 \\
\hline 1031612 & 0 & 2 & 2 & 2 & 3 & 1 & 2 & 1 & 1 & 1 & 1 & 1 & 1 & 1 & 3 & 1 & 1 & 0 & 1 \\
\hline 1031613 & 0 & 3 & 2 & 2 & 3 & 1 & 2 & 2 & 1 & 1 & 0 & 0 & 1 & 1 & 4 & 1 & 1 & 0 & 1 \\
\hline 1031614 & 0 & 3 & 2 & 2 & 3 & 1 & 1 & 1 & 1 & 1 & 0 & 0 & 1 & 1 & 2 & 1 & 1 & 0 & 1 \\
\hline 1031615 & 0 & 5 & 2 & 2 & 3 & 1 & 1 & 2 & 1 & 1 & 0 & 0 & 1 & 1 & 1 & 1 & 0 & 0 & 0 \\
\hline 1031616 & 0 & 4 & 2 & 2 & 3 & 1 & 1 & 2 & 1 & 1 & 0 & 0 & 0 & 99 & 99 & 99 & 99 & 99 & 99 \\
\hline 1031617 & 0 & 4 & 2 & 2 & 3 & 1 & 2 & 2 & 1 & 1 & 1 & 1 & 0 & 99 & 99 & 99 & 99 & 99 & 99 \\
\hline 1031618 & 0 & 4 & 2 & 2 & 3 & 1 & 1 & 2 & 1 & 1 & 0 & 0 & 0 & 99 & 99 & 99 & 99 & 99 & 99 \\
\hline 1031619 & 0 & 5 & 2 & 1 & 3 & 1 & 2 & 2 & 1 & 1 & 1 & 1 & 0 & 99 & 99 & 99 & 99 & 99 & 99 \\
\hline 1031620 & 0 & 3 & 2 & 3 & 3 & 1 & 2 & 1 & 1 & 1 & 0 & 0 & 0 & 99 & 99 & 99 & 99 & 99 & 99 \\
\hline 1031621 & 0 & 1 & 2 & 5 & 3 & 5 & 3 & 1 & 1 & 1 & 1 & 1 & 1 & 1 & 2 & 1 & 1 & 0 & 1 \\
\hline 1031622 & 0 & 4 & 2 & 4 & 3 & 1 & 2 & 2 & 1 & 1 & 1 & 1 & 0 & 99 & 99 & 99 & 99 & 99 & 99 \\
\hline 1031623 & 0 & 4 & 2 & 3 & 3 & 1 & 3 & 1 & 1 & 1 & 1 & 1 & 1 & 1 & 1 & 1 & 0 & 0 & 0 \\
\hline 204171 & 0 & 1 & 1 & 4 & 1 & 1 & 2 & 2 & 4 & 4 & 0 & 0 & 1 & 1 & 1 & 1 & 0 & 0 & 0 \\
\hline 204172 & 0 & 3 & 1 & 1 & 1 & 2 & 2 & 1 & 4 & 4 & 0 & 0 & 1 & 1 & 1 & 1 & 0 & 0 & 0 \\
\hline 204173 & 0 & 1 & 1 & 2 & 1 & 1 & 2 & 1 & 4 & 4 & 0 & 0 & 1 & 1 & 2 & 1 & 1 & 0 & \\
\hline
\end{tabular}




\begin{tabular}{|c|c|c|c|c|c|c|c|c|c|c|c|c|c|c|c|c|c|c|c|}
\hline 204174 & 1 & 2 & 1 & 2 & 1 & 2 & 2 & 2 & 4 & 4 & 0 & 0 & 1 & 1 & 3 & 1 & 1 & 0 & 1 \\
\hline 204175 & 0 & 1 & 1 & 4 & 1 & 2 & 2 & 1 & 4 & 4 & 0 & 0 & 1 & 1 & 2 & 1 & 1 & 0 & 1 \\
\hline 204176 & 1 & 5 & 1 & 2 & 1 & 2 & 2 & 2 & 4 & 4 & 0 & 0 & 1 & 1 & 2 & 1 & 1 & 0 & 1 \\
\hline 204177 & 1 & 4 & 1 & 2 & 1 & 2 & 1 & 2 & 4 & 1 & 0 & 0 & 1 & 1 & 1 & 4 & 0 & 1 & 1 \\
\hline 204178 & 1 & 2 & 1 & 1 & 1 & 2 & 2 & 1 & 4 & 4 & 0 & 0 & 1 & 1 & 3 & 1 & 1 & 0 & 1 \\
\hline 204179 & 0 & 1 & 1 & 2 & 1 & 1 & 2 & 2 & 4 & 2 & 0 & 0 & 1 & 1 & 3 & 1 & 1 & 0 & 1 \\
\hline 2041710 & 0 & 1 & 1 & 5 & 1 & 1 & 2 & 2 & 4 & 1 & 0 & 0 & 1 & 1 & 1 & 4 & 0 & 1 & 1 \\
\hline 2041711 & 1 & 4 & 1 & 2 & 1 & 2 & 2 & 2 & 4 & 4 & 0 & 0 & 1 & 1 & 3 & 4 & 1 & 1 & 1 \\
\hline 2041712 & 1 & 4 & 1 & 2 & 1 & 4 & 2 & 2 & 4 & 4 & 0 & 0 & 1 & 1 & 3 & 4 & 1 & 1 & 1 \\
\hline 2041713 & 0 & 4 & 1 & 2 & 1 & 1 & 2 & 1 & 4 & 4 & 0 & 0 & 1 & 1 & 1 & 4 & 0 & 1 & 1 \\
\hline 2041714 & 1 & 5 & 1 & 2 & 1 & 2 & 1 & 1 & 4 & 3 & 0 & 0 & 1 & 1 & 4 & 4 & 1 & 1 & 1 \\
\hline 2041715 & 1 & 5 & 1 & 2 & 1 & 2 & 2 & 1 & 4 & 1 & 0 & 0 & 1 & 1 & 4 & 4 & 1 & 1 & 1 \\
\hline 2041716 & 1 & 5 & 1 & 2 & 1 & 2 & 2 & 2 & 4 & 1 & 0 & 0 & 1 & 1 & 4 & 4 & 1 & 1 & 1 \\
\hline 2041717 & 1 & 4 & 1 & 3 & 1 & 5 & 3 & 2 & 4 & 1 & 1 & 1 & 1 & 1 & 1 & 1 & 0 & 0 & 0 \\
\hline 2041718 & 0 & 4 & 1 & 1 & 1 & 1 & 2 & 2 & 4 & 2 & 0 & 0 & 1 & 1 & 4 & 4 & 1 & 1 & 1 \\
\hline 2041719 & 0 & 3 & 1 & 2 & 1 & 1 & 2 & 2 & 4 & 1 & 0 & 0 & 1 & 1 & 3 & 1 & 1 & 0 & 1 \\
\hline 2041720 & 1 & 5 & 1 & 2 & 1 & 2 & 2 & 2 & 4 & 1 & 0 & 0 & 1 & 1 & 4 & 1 & 1 & 0 & 1 \\
\hline 2041721 & 1 & 4 & 1 & 1 & 1 & 2 & 2 & 2 & 4 & 1 & 0 & 0 & 1 & 1 & 3 & 4 & 1 & 1 & 1 \\
\hline 2041722 & 1 & 2 & 1 & 1 & 1 & 2 & 2 & 2 & 4 & 1 & 0 & 0 & 1 & 1 & 3 & 4 & 1 & 1 & 1 \\
\hline 2041723 & 0 & 2 & 1 & 3 & 1 & 1 & 2 & 2 & 4 & 1 & 0 & 0 & 1 & 1 & 1 & 1 & 0 & 0 & 0 \\
\hline 2041724 & 0 & 5 & 1 & 1 & 1 & 1 & 2 & 1 & 4 & 1 & 0 & 0 & 1 & 1 & 3 & 4 & 1 & 1 & 1 \\
\hline 2041725 & 1 & 5 & 1 & 1 & 1 & 2 & 2 & 2 & 4 & 2 & 0 & 0 & 1 & 1 & 1 & 4 & 0 & 1 & 1 \\
\hline 2041726 & 1 & 2 & 1 & 2 & 1 & 2 & 2 & 1 & 4 & 1 & 0 & 0 & 1 & 1 & 3 & 1 & 1 & 0 & 1 \\
\hline 2041727 & 1 & 5 & 1 & 1 & 1 & 2 & 2 & 2 & 4 & 2 & 0 & 0 & 1 & 1 & 3 & 1 & 1 & 0 & 1 \\
\hline 2041728 & 1 & 3 & 1 & 2 & 1 & 2 & 3 & 2 & 4 & 1 & 0 & 0 & 1 & 1 & 1 & 1 & 0 & 0 & 0 \\
\hline 2041729 & 1 & 2 & 1 & 2 & 1 & 2 & 2 & 2 & 4 & 3 & 0 & 0 & 1 & 1 & 3 & 4 & 1 & 1 & 1 \\
\hline 2041730 & 0 & 4 & 1 & 2 & 1 & 2 & 1 & 1 & 4 & 3 & 0 & 0 & 1 & 1 & 4 & 4 & 1 & 1 & 1 \\
\hline 2041731 & 1 & 1 & 1 & 2 & 1 & 2 & 2 & 1 & 4 & 3 & 0 & 0 & 1 & 1 & 1 & 1 & 0 & 0 & 0 \\
\hline 2041732 & 1 & 2 & 1 & 5 & 1 & 2 & 2 & 1 & 4 & 4 & 0 & 0 & 1 & 1 & 2 & 1 & 1 & 0 & 1 \\
\hline 2041733 & 1 & 2 & 1 & 3 & 1 & 2 & 2 & 1 & 4 & 4 & 0 & 0 & 1 & 1 & 2 & 1 & 1 & 0 & 1 \\
\hline 2041734 & 1 & 4 & 1 & 2 & 1 & 2 & 1 & 1 & 4 & 4 & 0 & 0 & 1 & 1 & 3 & 1 & 1 & 0 & 1 \\
\hline 2041735 & 0 & 3 & 1 & 2 & 1 & 1 & 2 & 2 & 4 & 1 & 0 & 0 & 1 & 1 & 2 & 4 & 1 & 1 & 1 \\
\hline 204181 & 1 & 3 & 1 & 2 & 1 & 2 & 3 & 2 & 1 & 2 & 0 & 0 & 1 & 1 & 3 & 4 & 1 & 1 & 1 \\
\hline 204182 & 1 & 4 & 1 & 2 & 1 & 2 & 1 & 2 & 1 & 1 & 0 & 0 & 1 & 1 & 3 & 1 & 1 & 0 & 1 \\
\hline 204183 & 1 & 3 & 1 & 2 & 1 & 2 & 2 & 2 & 1 & 3 & 0 & 0 & 1 & 1 & 4 & 4 & 1 & 1 & 1 \\
\hline 204184 & 1 & 4 & 1 & 2 & 1 & 2 & 2 & 1 & 1 & 2 & 0 & 0 & 1 & 1 & 4 & 4 & 1 & 1 & 1 \\
\hline 204185 & 0 & 5 & 1 & 1 & 1 & 2 & 2 & 1 & 1 & 1 & 0 & 0 & 1 & 1 & 3 & 1 & 1 & 0 & 1 \\
\hline 204186 & 0 & 5 & 1 & 1 & 1 & 2 & 2 & 1 & 2 & 1 & 0 & 0 & 1 & 1 & 3 & 4 & 1 & 1 & 1 \\
\hline 204187 & 1 & 2 & 1 & 5 & 1 & 2 & 2 & 2 & 4 & 2 & 0 & 0 & 1 & 1 & 1 & 1 & 0 & 0 & 0 \\
\hline 204188 & 1 & 1 & 1 & 4 & 1 & 2 & 2 & 1 & 1 & 2 & 0 & 0 & 1 & 1 & 3 & 1 & 1 & 0 & 1 \\
\hline 204189 & 1 & 4 & 1 & 2 & 1 & 2 & 2 & 1 & 1 & 1 & 0 & 0 & 1 & 1 & 3 & 4 & 1 & 1 & 1 \\
\hline 2041810 & 1 & 5 & 1 & 3 & 1 & 2 & 1 & 2 & 1 & 2 & 0 & 0 & 1 & 1 & 1 & 1 & 0 & 0 & 0 \\
\hline 2041811 & 1 & 4 & 1 & 4 & 1 & 2 & 3 & 2 & 4 & 2 & 0 & 0 & 1 & 1 & 3 & 4 & 1 & 1 & 1 \\
\hline 2041812 & 0 & 3 & 1 & 2 & 1 & 2 & 3 & 1 & 1 & 1 & 0 & 0 & 1 & 1 & 1 & 6 & 0 & 1 & 1 \\
\hline 2041813 & 0 & 3 & 1 & 2 & 1 & 2 & 2 & 1 & 4 & 2 & 0 & 0 & 1 & 1 & 4 & 1 & 1 & 0 & 1 \\
\hline 2041814 & 1 & 3 & 1 & 4 & 1 & 2 & 2 & 1 & 3 & 4 & 0 & 0 & 1 & 1 & 1 & 1 & 0 & 0 & 0 \\
\hline 2041815 & 1 & 1 & 1 & 5 & 1 & 2 & 1 & 1 & 4 & 4 & 0 & 0 & 1 & 1 & 3 & 1 & 1 & 0 & 1 \\
\hline 2041816 & 1 & 3 & 1 & 2 & 1 & 2 & 2 & 1 & 4 & 4 & 0 & 0 & 1 & 1 & 3 & 1 & 1 & 0 & 1 \\
\hline 2041817 & 1 & 4 & 1 & 2 & 1 & 2 & 2 & 2 & 4 & 4 & 0 & 0 & 1 & 1 & 1 & 1 & 0 & 0 & 0 \\
\hline 2041818 & 1 & 5 & 1 & 2 & 1 & 2 & 2 & 2 & 1 & 1 & 0 & 0 & 1 & 1 & 3 & 1 & 1 & 0 & 1 \\
\hline 2041819 & 1 & 2 & 1 & 5 & 1 & 5 & 2 & 1 & 4 & 4 & 1 & 1 & 1 & 1 & 1 & 1 & 0 & 0 & 0 \\
\hline 2041820 & 0 & 4 & 1 & 1 & 1 & 2 & 3 & 2 & 1 & 1 & 0 & 0 & 1 & 1 & 4 & 1 & 1 & 0 & 1 \\
\hline 2041821 & 1 & 3 & 1 & 3 & 1 & 2 & 2 & 2 & 4 & 2 & 0 & 0 & 1 & 1 & 3 & 1 & 1 & 0 & 1 \\
\hline 2041822 & 1 & 4 & 1 & 1 & 1 & 2 & 2 & 1 & 4 & 4 & 0 & 0 & 1 & 1 & 2 & 1 & 1 & 0 & 1 \\
\hline 2041823 & 1 & 5 & 1 & 1 & 1 & 2 & 2 & 2 & 1 & 1 & 0 & 0 & 1 & 1 & 3 & 1 & 1 & 0 & 1 \\
\hline 2041824 & 1 & 1 & 1 & 4 & 1 & 2 & 2 & 1 & 1 & 1 & 0 & 0 & 1 & 1 & 1 & 4 & 0 & 1 & \\
\hline
\end{tabular}




\begin{tabular}{|c|c|c|c|c|c|c|c|c|c|c|c|c|c|c|c|c|c|c|c|}
\hline 2041825 & 0 & 1 & 1 & 3 & 1 & 1 & 2 & 1 & 1 & 1 & 0 & 0 & 1 & 1 & 3 & 4 & 1 & 1 & 1 \\
\hline 2041826 & 1 & 3 & 1 & 2 & 1 & 2 & 2 & 2 & 1 & 1 & 0 & 0 & 1 & 1 & 3 & 4 & 1 & 1 & 1 \\
\hline 2041827 & 1 & 5 & 1 & 2 & 1 & 2 & 2 & 1 & 1 & 1 & 0 & 0 & 1 & 1 & 3 & 4 & 1 & 1 & 1 \\
\hline 2041828 & 1 & 5 & 1 & 1 & 1 & 2 & 2 & 2 & 4 & 4 & 0 & 0 & 1 & 1 & 3 & 4 & 1 & 1 & 1 \\
\hline 2041829 & 1 & 1 & 1 & 4 & 1 & 2 & 2 & 1 & 4 & 4 & 0 & 0 & 1 & 1 & 4 & 1 & 1 & 0 & 1 \\
\hline 2041830 & 1 & 4 & 1 & 2 & 1 & 1 & 2 & 2 & 1 & 1 & 0 & 0 & 1 & 1 & 4 & 1 & 1 & 0 & 1 \\
\hline 204191 & 1 & 2 & 1 & 4 & 1 & 2 & 2 & 2 & 3 & 4 & 0 & 0 & 1 & 1 & 1 & 5 & 0 & 1 & 1 \\
\hline 204192 & 1 & 1 & 1 & 5 & 1 & 2 & 2 & 1 & 1 & 2 & 0 & 0 & 1 & 1 & 3 & 1 & 1 & 0 & 1 \\
\hline 204193 & 0 & 2 & 1 & 2 & 1 & 2 & 1 & 1 & 3 & 4 & 0 & 0 & 1 & 1 & 3 & 1 & 1 & 0 & 1 \\
\hline 204194 & 1 & 5 & 1 & 1 & 1 & 2 & 1 & 1 & 3 & 4 & 0 & 0 & 1 & 1 & 3 & 7 & 1 & 1 & 1 \\
\hline 204195 & 0 & 3 & 1 & 2 & 1 & 2 & 2 & 2 & 1 & 3 & 0 & 0 & 1 & 1 & 1 & 5 & 0 & 1 & 1 \\
\hline 204196 & 0 & 4 & 1 & 2 & 1 & 2 & 1 & 1 & 1 & 2 & 0 & 0 & 1 & 1 & 3 & 1 & 1 & 0 & 1 \\
\hline 204197 & 0 & 5 & 1 & 1 & 1 & 2 & 1 & 1 & 1 & 2 & 0 & 0 & 1 & 1 & 2 & 1 & 1 & 0 & 1 \\
\hline 204198 & 0 & 3 & 1 & 4 & 1 & 1 & 2 & 1 & 1 & 2 & 0 & 0 & 1 & 1 & 3 & 7 & 1 & 1 & 1 \\
\hline 204199 & 0 & 5 & 1 & 1 & 1 & 2 & 1 & 1 & 1 & 4 & 0 & 0 & 0 & 99 & 99 & 99 & 99 & 99 & 99 \\
\hline 2041910 & 0 & 3 & 1 & 2 & 1 & 2 & 1 & 2 & 3 & 4 & 0 & 0 & 1 & 1 & 3 & 5 & 1 & 1 & 1 \\
\hline 2041911 & 0 & 3 & 1 & 2 & 1 & 2 & 2 & 2 & 1 & 2 & 0 & 0 & 1 & 1 & 2 & 7 & 1 & 1 & 1 \\
\hline 2041912 & 1 & 2 & 1 & 3 & 1 & 2 & 2 & 1 & 3 & 4 & 0 & 0 & 1 & 1 & 3 & 1 & 1 & 0 & 1 \\
\hline 2041913 & 1 & 5 & 1 & 1 & 1 & 2 & 1 & 1 & 3 & 4 & 0 & 0 & 1 & 1 & 3 & 6 & 1 & 1 & 1 \\
\hline 2041914 & 1 & 5 & 1 & 1 & 1 & 2 & 1 & 1 & 3 & 4 & 0 & 0 & 1 & 1 & 3 & 1 & 1 & 0 & 1 \\
\hline 2041915 & 0 & 3 & 1 & 2 & 1 & 2 & 1 & 1 & 3 & 2 & 0 & 0 & 1 & 1 & 3 & 1 & 1 & 0 & 1 \\
\hline 2041916 & 1 & 2 & 1 & 2 & 1 & 2 & 2 & 1 & 3 & 4 & 0 & 0 & 1 & 1 & 3 & 1 & 1 & 0 & 1 \\
\hline 2041917 & 1 & 2 & 1 & 2 & 1 & 2 & 1 & 2 & 3 & 4 & 0 & 0 & 1 & 1 & 2 & 1 & 1 & 0 & 1 \\
\hline 2041918 & 1 & 3 & 1 & 2 & 1 & 2 & 2 & 2 & 3 & 3 & 0 & 0 & 1 & 1 & 3 & 1 & 1 & 0 & 1 \\
\hline 2041919 & 1 & 3 & 1 & 2 & 1 & 2 & 1 & 1 & 3 & 4 & 0 & 0 & 1 & 1 & 3 & 1 & 1 & 0 & 1 \\
\hline 2041920 & 0 & 5 & 1 & 1 & 1 & 2 & 1 & 1 & 3 & 4 & 0 & 0 & 1 & 1 & 2 & 1 & 1 & 0 & 1 \\
\hline 2041921 & 0 & 3 & 1 & 2 & 1 & 2 & 2 & 2 & 3 & 4 & 0 & 0 & 1 & 1 & 1 & 1 & 0 & 0 & 0 \\
\hline 2041922 & 1 & 4 & 1 & 2 & 1 & 2 & 1 & 1 & 3 & 3 & 0 & 0 & 1 & 1 & 2 & 1 & 1 & 0 & 1 \\
\hline 2041923 & 1 & 5 & 1 & 1 & 1 & 2 & 1 & 1 & 3 & 4 & 0 & 0 & 1 & 1 & 2 & 6 & 1 & 1 & 1 \\
\hline 2041924 & 1 & 2 & 1 & 2 & 1 & 2 & 2 & 2 & 3 & 4 & 0 & 0 & 1 & 1 & 3 & 1 & 1 & 0 & 1 \\
\hline 2041925 & 1 & 2 & 1 & 2 & 1 & 2 & 1 & 1 & 3 & 4 & 0 & 0 & 1 & 1 & 1 & 1 & 0 & 0 & 0 \\
\hline 2041926 & 0 & 2 & 1 & 3 & 1 & 2 & 1 & 1 & 3 & 4 & 0 & 0 & 1 & 1 & 1 & 1 & 0 & 0 & 0 \\
\hline 2041927 & 1 & 4 & 1 & 1 & 1 & 2 & 2 & 2 & 3 & 4 & 0 & 0 & 1 & 1 & 2 & 1 & 1 & 0 & 1 \\
\hline 2041928 & 0 & 4 & 1 & 1 & 1 & 2 & 1 & 1 & 3 & 4 & 0 & 0 & 1 & 1 & 1 & 1 & 0 & 0 & 0 \\
\hline 2041929 & 0 & 1 & 1 & 4 & 1 & 2 & 1 & 1 & 3 & 4 & 0 & 0 & 1 & 1 & 2 & 1 & 1 & 0 & 1 \\
\hline 2041930 & 1 & 2 & 1 & 2 & 1 & 2 & 1 & 1 & 1 & 4 & 0 & 0 & 1 & 1 & 2 & 1 & 1 & 0 & 1 \\
\hline 204201 & 1 & 2 & 1 & 4 & 1 & 2 & 2 & 1 & 1 & 4 & 0 & 0 & 1 & 1 & 3 & 7 & 1 & 1 & 1 \\
\hline 204202 & 0 & 1 & 1 & 4 & 1 & 2 & 2 & 1 & 1 & 4 & 0 & 0 & 1 & 1 & 3 & 1 & 1 & 0 & 1 \\
\hline 204203 & 1 & 3 & 1 & 5 & 1 & 5 & 2 & 1 & 1 & 4 & 0 & 0 & 1 & 1 & 3 & 1 & 1 & 0 & 1 \\
\hline 204204 & 1 & 2 & 1 & 2 & 1 & 2 & 1 & 1 & 1 & 4 & 0 & 0 & 1 & 1 & 3 & 7 & 1 & 1 & 1 \\
\hline 204205 & 1 & 3 & 1 & 2 & 1 & 2 & 2 & 2 & 1 & 2 & 0 & 0 & 1 & 1 & 2 & 1 & 1 & 0 & 1 \\
\hline 204206 & 1 & 5 & 1 & 1 & 1 & 2 & 1 & 1 & 1 & 2 & 0 & 0 & 0 & 99 & 99 & 99 & 99 & 99 & 99 \\
\hline 204207 & 1 & 3 & 1 & 2 & 1 & 2 & 1 & 2 & 1 & 1 & 0 & 0 & 1 & 1 & 3 & 1 & 1 & 0 & 1 \\
\hline 204208 & 1 & 1 & 1 & 3 & 1 & 2 & 2 & 1 & 1 & 2 & 0 & 0 & 1 & 1 & 3 & 1 & 1 & 0 & 1 \\
\hline 204209 & 0 & 3 & 1 & 2 & 1 & 1 & 1 & 1 & 1 & 2 & 0 & 0 & 1 & 1 & 2 & 1 & 1 & 0 & 1 \\
\hline 2042010 & 0 & 3 & 1 & 2 & 1 & 3 & 1 & 2 & 1 & 2 & 0 & 0 & 1 & 1 & 3 & 1 & 1 & 0 & 1 \\
\hline 2042011 & 1 & 5 & 1 & 1 & 1 & 2 & 1 & 1 & 1 & 3 & 0 & 0 & 1 & 1 & 2 & 1 & 1 & 0 & 1 \\
\hline 2042012 & 0 & 5 & 1 & 1 & 1 & 2 & 1 & 1 & 1 & 3 & 0 & 0 & 0 & 99 & 99 & 99 & 99 & 99 & 99 \\
\hline 2042013 & 1 & 3 & 1 & 2 & 1 & 2 & 2 & 2 & 1 & 4 & 0 & 0 & 1 & 1 & 3 & 1 & 1 & 0 & 1 \\
\hline 2042014 & 0 & 4 & 1 & 1 & 1 & 2 & 1 & 2 & 1 & 4 & 0 & 0 & 1 & 1 & 2 & 1 & 1 & 0 & 1 \\
\hline 2042015 & 1 & 2 & 1 & 2 & 1 & 2 & 2 & 1 & 1 & 2 & 0 & 0 & 1 & 1 & 2 & 1 & 1 & 0 & 1 \\
\hline 2042016 & 0 & 2 & 1 & 2 & 1 & 1 & 1 & 1 & 1 & 2 & 0 & 0 & 1 & 1 & 3 & 6 & 1 & 1 & 1 \\
\hline 2042017 & 0 & 2 & 1 & 2 & 1 & 2 & 3 & 2 & 1 & 1 & 0 & 0 & 1 & 1 & 3 & 6 & 1 & 1 & 1 \\
\hline 2042018 & 1 & 4 & 1 & 2 & 1 & 2 & 2 & 2 & 4 & 4 & 0 & 0 & 1 & 1 & 3 & 1 & 1 & 0 & 1 \\
\hline 2042019 & 1 & 3 & 1 & 2 & 1 & 2 & 2 & 2 & 1 & 4 & 0 & 0 & 1 & 1 & 3 & 1 & 1 & 0 & 1 \\
\hline 2042020 & 0 & 5 & 1 & 1 & 1 & 2 & 1 & 1 & 1 & 4 & 0 & 0 & 1 & 1 & 3 & 1 & 1 & 0 & 1 \\
\hline
\end{tabular}




\begin{tabular}{|c|c|c|c|c|c|c|c|c|c|c|c|c|c|c|c|c|c|c|c|}
\hline 2042021 & 1 & 3 & 1 & 2 & 1 & 2 & 2 & 1 & 1 & 4 & 0 & 0 & 1 & 1 & 4 & 1 & 1 & 0 & 1 \\
\hline 2042022 & 1 & 5 & 1 & 1 & 1 & 2 & 1 & 1 & 1 & 4 & 0 & 0 & 1 & 1 & 2 & 6 & 1 & 1 & 1 \\
\hline 2042023 & 1 & 2 & 1 & 2 & 1 & 2 & 2 & 2 & 1 & 4 & 0 & 0 & 1 & 1 & 1 & 1 & 0 & 0 & 0 \\
\hline 2042024 & 1 & 1 & 1 & 4 & 1 & 2 & 2 & 1 & 1 & 4 & 0 & 0 & 1 & 1 & 4 & 1 & 1 & 0 & 1 \\
\hline 2042025 & 1 & 3 & 1 & 2 & 1 & 2 & 1 & 2 & 1 & 4 & 0 & 0 & 1 & 1 & 3 & 1 & 1 & 0 & 1 \\
\hline 205211 & 0 & 3 & 1 & 2 & 1 & 2 & 1 & 2 & 3 & 1 & 0 & 0 & 1 & 1 & 4 & 4 & 1 & 1 & 1 \\
\hline 205212 & 1 & 3 & 1 & 2 & 1 & 2 & 3 & 2 & 3 & 1 & 0 & 0 & 1 & 1 & 1 & 1 & 0 & 0 & 0 \\
\hline 205213 & 0 & 5 & 1 & 2 & 1 & 2 & 1 & 2 & 3 & 1 & 0 & 0 & 1 & 1 & 2 & 1 & 1 & 0 & 1 \\
\hline 205214 & 0 & 3 & 1 & 2 & 1 & 2 & 1 & 1 & 3 & 2 & 0 & 0 & 1 & 1 & 2 & 1 & 1 & 0 & 1 \\
\hline 205215 & 1 & 4 & 1 & 4 & 1 & 2 & 1 & 1 & 3 & 3 & 0 & 0 & 1 & 1 & 2 & 1 & 1 & 0 & 1 \\
\hline 205216 & 0 & 4 & 1 & 1 & 1 & 2 & 1 & 1 & 3 & 3 & 0 & 0 & 1 & 1 & 3 & 1 & 1 & 0 & 1 \\
\hline 205217 & 0 & 2 & 1 & 2 & 1 & 2 & 1 & 2 & 3 & 3 & 0 & 0 & 1 & 1 & 2 & 1 & 1 & 0 & 1 \\
\hline 205218 & 1 & 2 & 1 & 4 & 1 & 2 & 1 & 2 & 3 & 3 & 0 & 0 & 1 & 1 & 1 & 1 & 0 & 0 & 0 \\
\hline 205219 & 1 & 5 & 1 & 2 & 1 & 2 & 1 & 2 & 3 & 4 & 0 & 0 & 1 & 1 & 1 & 1 & 0 & 0 & 0 \\
\hline 2052110 & 0 & 3 & 1 & 2 & 1 & 2 & 2 & 2 & 3 & 2 & 0 & 0 & 1 & 1 & 1 & 1 & 0 & 0 & 0 \\
\hline 2052111 & 0 & 4 & 1 & 2 & 1 & 2 & 1 & 1 & 3 & 1 & 0 & 0 & 1 & 1 & 2 & 1 & 1 & 0 & 1 \\
\hline 2052112 & 1 & 3 & 1 & 2 & 1 & 2 & 2 & 1 & 3 & 3 & 0 & 0 & 1 & 1 & 2 & 1 & 1 & 0 & 1 \\
\hline 2052113 & 0 & 3 & 1 & 1 & 1 & 2 & 2 & 1 & 3 & 2 & 0 & 0 & 1 & 1 & 2 & 1 & 1 & 0 & 1 \\
\hline 2052114 & 0 & 2 & 1 & 2 & 1 & 2 & 1 & 2 & 3 & 2 & 0 & 0 & 1 & 1 & 1 & 1 & 0 & 0 & 0 \\
\hline 2052115 & 0 & 1 & 1 & 5 & 1 & 5 & 2 & 1 & 3 & 1 & 0 & 0 & 1 & 1 & 1 & 1 & 0 & 0 & 0 \\
\hline 2052116 & 1 & 3 & 1 & 2 & 1 & 2 & 1 & 2 & 3 & 4 & 0 & 0 & 1 & 1 & 3 & 1 & 1 & 0 & 1 \\
\hline 2052117 & 1 & 2 & 1 & 2 & 1 & 2 & 2 & 1 & 3 & 1 & 0 & 0 & 1 & 1 & 1 & 1 & 0 & 0 & 0 \\
\hline 2052118 & 0 & 3 & 1 & 2 & 1 & 2 & 1 & 2 & 3 & 1 & 0 & 0 & 1 & 1 & 5 & 4 & 1 & 1 & 1 \\
\hline 2052119 & 0 & 3 & 1 & 2 & 1 & 2 & 1 & 2 & 3 & 1 & 0 & 0 & 1 & 1 & 1 & 1 & 0 & 0 & 0 \\
\hline 2052120 & 1 & 5 & 1 & 1 & 1 & 2 & 1 & 1 & 3 & 1 & 0 & 0 & 1 & 1 & 2 & 1 & 1 & 0 & 1 \\
\hline 2052121 & 0 & 3 & 1 & 2 & 1 & 2 & 2 & 2 & 3 & 1 & 0 & 0 & 1 & 1 & 3 & 1 & 1 & 0 & 1 \\
\hline 2052122 & 1 & 3 & 1 & 2 & 1 & 2 & 1 & 2 & 1 & 1 & 0 & 0 & 0 & 99 & 99 & 99 & 99 & 99 & 99 \\
\hline 2052123 & 0 & 3 & 1 & 2 & 1 & 2 & 2 & 2 & 3 & 1 & 0 & 0 & 1 & 1 & 1 & 1 & 0 & 0 & 0 \\
\hline 2052124 & 0 & 3 & 1 & 2 & 1 & 2 & 1 & 2 & 3 & 1 & 0 & 0 & 1 & 1 & 2 & 1 & 1 & 0 & 1 \\
\hline 2052125 & 1 & 2 & 1 & 2 & 1 & 2 & 1 & 2 & 1 & 4 & 0 & 0 & 1 & 1 & 2 & 1 & 1 & 0 & 1 \\
\hline 2052126 & 1 & 2 & 1 & 4 & 1 & 2 & 2 & 2 & 3 & 2 & 0 & 0 & 1 & 1 & 1 & 1 & 0 & 0 & 0 \\
\hline 2052127 & 0 & 3 & 1 & 2 & 1 & 2 & 1 & 2 & 3 & 2 & 0 & 0 & 1 & 1 & 2 & 1 & 1 & 0 & 1 \\
\hline 2052128 & 0 & 3 & 1 & 3 & 2 & 2 & 2 & 2 & 3 & 2 & 0 & 0 & 1 & 1 & 1 & 1 & 0 & 0 & 0 \\
\hline 2052129 & 1 & 4 & 1 & 2 & 1 & 2 & 2 & 1 & 2 & 1 & 0 & 0 & 1 & 1 & 3 & 1 & 1 & 0 & 1 \\
\hline 2052130 & 0 & 5 & 1 & 1 & 1 & 2 & 1 & 1 & 3 & 3 & 0 & 0 & 0 & 99 & 99 & 99 & 99 & 99 & 99 \\
\hline 205221 & 0 & 2 & 1 & 1 & 1 & 2 & 2 & 2 & 4 & 4 & 0 & 0 & 1 & 1 & 1 & 1 & 0 & 0 & 0 \\
\hline 205222 & 1 & 3 & 1 & 1 & 1 & 2 & 2 & 2 & 4 & 4 & 0 & 0 & 1 & 1 & 2 & 1 & 1 & 0 & 1 \\
\hline 205223 & 1 & 1 & 1 & 1 & 1 & 2 & 1 & 2 & 4 & 4 & 0 & 0 & 1 & 1 & 3 & 1 & 1 & 0 & 1 \\
\hline 205224 & 0 & 1 & 1 & 1 & 2 & 3 & 2 & 1 & 4 & 4 & 0 & 0 & 1 & 1 & 2 & 7 & 1 & 1 & 1 \\
\hline 205225 & 1 & 2 & 1 & 3 & 1 & 2 & 1 & 2 & 4 & 4 & 0 & 0 & 1 & 1 & 3 & 1 & 1 & 0 & 1 \\
\hline 205226 & 1 & 3 & 1 & 2 & 1 & 2 & 1 & 1 & 4 & 4 & 0 & 0 & 1 & 1 & 3 & 1 & 1 & 0 & 1 \\
\hline 205227 & 0 & 4 & 1 & 1 & 1 & 2 & 2 & 2 & 4 & 4 & 0 & 0 & 1 & 1 & 3 & 1 & 1 & 0 & 1 \\
\hline 205228 & 0 & 4 & 1 & 1 & 1 & 2 & 1 & 2 & 3 & 4 & 0 & 0 & 1 & 1 & 2 & 1 & 1 & 0 & 1 \\
\hline 205229 & 0 & 1 & 1 & 4 & 1 & 2 & 2 & 2 & 3 & 2 & 0 & 0 & 1 & 1 & 2 & 1 & 1 & 0 & 1 \\
\hline 2052210 & 1 & 5 & 1 & 1 & 1 & 4 & 2 & 2 & 3 & 1 & 0 & 0 & 1 & 1 & 2 & 1 & 1 & 0 & 1 \\
\hline 2052211 & 0 & 3 & 1 & 2 & 1 & 2 & 2 & 2 & 3 & 1 & 0 & 0 & 1 & 1 & 1 & 1 & 0 & 0 & 0 \\
\hline 2052212 & 0 & 1 & 1 & 4 & 1 & 2 & 2 & 1 & 3 & 1 & 0 & 0 & 1 & 1 & 1 & 1 & 0 & 0 & 0 \\
\hline 2052213 & 0 & 4 & 1 & 3 & 2 & 1 & 2 & 2 & 3 & 2 & 0 & 0 & 1 & 1 & 2 & 1 & 1 & 0 & 1 \\
\hline 2052214 & 0 & 4 & 1 & 2 & 2 & 1 & 2 & 2 & 3 & 2 & 0 & 0 & 1 & 1 & 2 & 1 & 1 & 0 & 1 \\
\hline 2052215 & 0 & 3 & 1 & 3 & 1 & 2 & 2 & 2 & 3 & 1 & 0 & 0 & 1 & 1 & 1 & 1 & 0 & 0 & 0 \\
\hline 2052216 & 0 & 2 & 1 & 1 & 1 & 2 & 1 & 2 & 3 & 2 & 0 & 0 & 1 & 1 & 1 & 2 & 0 & 0 & 0 \\
\hline 2052217 & 0 & 2 & 1 & 2 & 1 & 2 & 1 & 2 & 3 & 3 & 0 & 0 & 1 & 1 & 1 & 1 & 0 & 0 & 0 \\
\hline 2052218 & 0 & 1 & 1 & 2 & 1 & 2 & 1 & 1 & 3 & 4 & 0 & 0 & 1 & 1 & 2 & 1 & 1 & 0 & 1 \\
\hline 2052219 & 0 & 5 & 1 & 1 & 1 & 2 & 1 & 2 & 3 & 4 & 0 & 0 & 1 & 1 & 2 & 1 & 1 & 0 & 1 \\
\hline 2052220 & 0 & 2 & 1 & 2 & 1 & 2 & 2 & 2 & 3 & 4 & 0 & 0 & 1 & 1 & 3 & 1 & 1 & 0 & 1 \\
\hline 2052221 & 0 & 3 & 1 & 2 & 1 & 2 & 2 & 2 & 3 & 4 & 0 & 0 & 1 & 1 & 3 & 1 & 1 & 0 & \\
\hline
\end{tabular}




\begin{tabular}{|c|c|c|c|c|c|c|c|c|c|c|c|c|c|c|c|c|c|c|c|}
\hline 2052222 & 0 & 2 & 1 & 1 & 1 & 3 & 3 & 2 & 2 & 4 & 0 & 0 & 1 & 1 & 2 & 1 & 1 & 0 & 1 \\
\hline 2052223 & 0 & 2 & 1 & 1 & 1 & 2 & 1 & 2 & 3 & 4 & 0 & 0 & 1 & 1 & 2 & 1 & 1 & 0 & 1 \\
\hline 2052224 & 1 & 5 & 1 & 2 & 1 & 2 & 2 & 1 & 3 & 4 & 0 & 0 & 1 & 1 & 2 & 1 & 1 & 0 & 1 \\
\hline 2052225 & 0 & 3 & 1 & 1 & 1 & 2 & 2 & 1 & 3 & 1 & 0 & 0 & 1 & 1 & 2 & 1 & 1 & 0 & 1 \\
\hline 2052226 & 0 & 4 & 1 & 1 & 1 & 1 & 2 & 2 & 3 & 1 & 0 & 0 & 1 & 1 & 1 & 1 & \begin{tabular}{l|l}
0 \\
\end{tabular} & 0 & 0 \\
\hline 2052227 & 0 & 2 & 1 & 1 & 1 & 1 & 1 & 2 & 3 & 1 & 0 & 0 & 1 & 1 & 1 & 1 & 0 & 0 & 0 \\
\hline 2052228 & 0 & 1 & 1 & 1 & 1 & 2 & 1 & 2 & 4 & 2 & 0 & 0 & 1 & 1 & 2 & 1 & 1 & 0 & 1 \\
\hline 2052229 & 0 & 5 & 1 & 3 & 2 & 2 & 2 & 1 & 4 & 2 & 0 & 0 & 1 & 1 & 1 & 1 & 0 & 0 & 0 \\
\hline 2052230 & 0 & 2 & 1 & 1 & 1 & 2 & 1 & 2 & 3 & 1 & 0 & 0 & 1 & 1 & 2 & 1 & 1 & 0 & 1 \\
\hline 205231 & 0 & 1 & 1 & 5 & 1 & 5 & 2 & 1 & 3 & 1 & 0 & 0 & 1 & 1 & 2 & 1 & 1 & 0 & 1 \\
\hline 205232 & 0 & 3 & 1 & 1 & 1 & 1 & 2 & 2 & 3 & 1 & 0 & 0 & 1 & 1 & 3 & 1 & 1 & 0 & 1 \\
\hline 205233 & 0 & 1 & 1 & 4 & 1 & 2 & 2 & 2 & 3 & 1 & 0 & 0 & 1 & 1 & 1 & 1 & 0 & 0 & 0 \\
\hline 205234 & 0 & 3 & 1 & 4 & 1 & 2 & 2 & 1 & 3 & 1 & 0 & 0 & 1 & 1 & 2 & 1 & 1 & 0 & 1 \\
\hline 205235 & 1 & 2 & 1 & 3 & 1 & 3 & 3 & 1 & 3 & 1 & 1 & 0 & 1 & 1 & 4 & 1 & 1 & 0 & 1 \\
\hline 205236 & 0 & 3 & 1 & 2 & 1 & 2 & 2 & 1 & 3 & 1 & 0 & 0 & 1 & 1 & 2 & 1 & 1 & 0 & 1 \\
\hline 205237 & 0 & 2 & 1 & 2 & 1 & 3 & 2 & 2 & 4 & 2 & 0 & 0 & 1 & 1 & 1 & 1 & 0 & 0 & 0 \\
\hline 205238 & 1 & 3 & 1 & 1 & 1 & 2 & 2 & 2 & 4 & 3 & 0 & 0 & 1 & 1 & 2 & 1 & 1 & 0 & 1 \\
\hline 205239 & 0 & 1 & 1 & 3 & 2 & 1 & 1 & 2 & 4 & 4 & 0 & 0 & 1 & 1 & 3 & 1 & 1 & 0 & 1 \\
\hline 2052310 & 1 & 3 & 1 & 1 & 1 & 2 & 2 & 2 & 4 & 4 & 0 & 0 & 1 & 1 & 3 & 1 & 1 & 0 & 1 \\
\hline 2052311 & 1 & 2 & 1 & 1 & 1 & 4 & 1 & 2 & 4 & 2 & 0 & 0 & 1 & 1 & 2 & 1 & 1 & 0 & 1 \\
\hline 2052312 & 1 & 3 & 1 & 1 & 1 & 4 & 2 & 2 & 4 & 3 & 0 & 0 & 1 & 1 & 2 & 1 & 1 & 0 & 1 \\
\hline 2052313 & 0 & 3 & 1 & 2 & 1 & 2 & 2 & 1 & 4 & 4 & 0 & 0 & 1 & 1 & 2 & 1 & 1 & 0 & 1 \\
\hline 2052314 & 0 & 3 & 1 & 5 & 1 & 5 & 2 & 2 & 4 & 4 & 0 & 0 & 1 & 1 & 1 & 1 & 0 & 0 & 0 \\
\hline 2052315 & 0 & 2 & 1 & 1 & 1 & 2 & 2 & 2 & 4 & 2 & 0 & 0 & 1 & 1 & 2 & 1 & 1 & 0 & 1 \\
\hline 2052316 & 1 & 5 & 1 & 1 & 1 & 2 & 2 & 2 & 4 & 3 & 0 & 0 & 1 & 1 & 3 & 1 & 1 & 0 & 1 \\
\hline 2052317 & 0 & 1 & 1 & 1 & 1 & 2 & 2 & 1 & 4 & 4 & 0 & 0 & 1 & 1 & 3 & 1 & 1 & 0 & 1 \\
\hline 2052318 & 1 & 5 & 1 & 4 & 1 & 5 & 3 & 2 & 4 & 4 & 1 & 1 & 1 & 1 & 1 & 1 & 0 & 0 & 0 \\
\hline 2052319 & 1 & 5 & 1 & 5 & 1 & 5 & 3 & 2 & 4 & 1 & 0 & 0 & 1 & 1 & 1 & 1 & 0 & 0 & 0 \\
\hline 2052320 & 0 & 2 & 1 & 2 & 1 & 3 & 3 & 2 & 3 & 1 & 1 & 1 & 1 & 1 & 2 & 1 & 1 & 0 & 1 \\
\hline 2052321 & 1 & 5 & 1 & 4 & 1 & 2 & 2 & 2 & 3 & 1 & 0 & 0 & 1 & 1 & 2 & 1 & 1 & 0 & 1 \\
\hline 2052322 & 0 & 4 & 1 & 1 & 1 & 2 & 2 & 2 & 3 & 1 & 0 & 0 & 1 & 1 & 3 & 1 & 1 & 0 & 1 \\
\hline 2052323 & 1 & 5 & 1 & 1 & 1 & 2 & 2 & 2 & 3 & 1 & 0 & 0 & 1 & 1 & 4 & 1 & 1 & 0 & 1 \\
\hline 2052324 & 0 & 1 & 1 & 1 & 1 & 2 & 1 & 2 & 3 & 1 & 0 & 0 & 1 & 1 & 2 & 1 & 1 & 0 & 1 \\
\hline 2052325 & 1 & 3 & 1 & 1 & 1 & 2 & 2 & 2 & 3 & 1 & 0 & 0 & 1 & 1 & 3 & 1 & 1 & 0 & 1 \\
\hline 2052326 & 0 & 1 & 1 & 2 & 1 & 2 & 2 & 2 & 3 & 1 & 0 & 0 & 1 & 1 & 3 & 1 & 1 & 0 & 1 \\
\hline 2052327 & 1 & 5 & 1 & 1 & 1 & 2 & 1 & 1 & 3 & 1 & 0 & 0 & 1 & 1 & 2 & 1 & 1 & 0 & 1 \\
\hline 2052328 & 1 & 3 & 1 & 2 & 1 & 2 & 2 & 2 & 3 & 1 & 0 & 0 & 1 & 1 & 1 & 1 & 0 & 0 & 0 \\
\hline 2052329 & 1 & 1 & 1 & 1 & 1 & 2 & 2 & 1 & 4 & 1 & 0 & 0 & 1 & 1 & 2 & 6 & 1 & 1 & 1 \\
\hline 2052330 & 0 & 1 & 1 & 1 & 1 & 1 & 1 & 2 & 4 & 1 & 0 & 0 & 1 & 1 & 1 & 1 & 0 & 0 & 0 \\
\hline 205241 & 0 & 1 & 1 & 4 & 2 & 1 & 2 & 1 & 4 & 4 & 0 & 0 & 1 & 1 & 1 & 1 & 0 & 0 & 0 \\
\hline 205242 & 0 & 3 & 1 & 2 & 1 & 2 & 3 & 2 & 4 & 3 & 0 & 0 & 1 & 1 & 1 & 6 & 0 & 1 & 1 \\
\hline 205243 & 0 & 3 & 1 & 4 & 2 & 1 & 3 & 1 & 4 & 4 & 1 & 1 & 1 & 1 & 1 & 1 & 0 & 0 & 0 \\
\hline 205244 & 0 & 3 & 1 & 3 & 2 & 1 & 3 & 2 & 4 & 3 & 1 & 1 & 1 & 1 & 1 & 1 & 0 & 0 & 0 \\
\hline 205245 & 1 & 3 & 1 & 4 & 2 & 2 & 2 & 2 & 4 & 4 & 1 & 1 & 1 & 1 & 1 & 1 & 0 & 0 & 0 \\
\hline 205246 & 0 & 4 & 1 & 5 & 2 & 5 & 3 & 2 & 4 & 4 & 1 & 1 & 1 & 1 & 1 & 1 & 0 & 0 & 0 \\
\hline 205247 & 1 & 4 & 1 & 3 & 1 & 2 & 3 & 2 & 4 & 4 & 0 & 0 & 1 & 1 & 1 & 1 & 0 & 0 & 0 \\
\hline 205248 & 1 & 3 & 1 & 2 & 1 & 4 & 2 & 2 & 4 & 4 & 0 & 0 & 1 & 1 & 2 & 5 & 1 & 1 & 1 \\
\hline 205249 & 0 & 2 & 1 & 2 & 2 & 1 & 2 & 2 & 4 & 4 & 0 & 0 & 1 & 1 & 3 & 1 & 1 & 0 & 1 \\
\hline 2052410 & 0 & 2 & 1 & 2 & 1 & 1 & 3 & 2 & 4 & 4 & 0 & 0 & 1 & 1 & 3 & 1 & 1 & 0 & 1 \\
\hline 2052411 & 1 & 2 & 1 & 1 & 1 & 4 & 3 & 1 & 4 & 4 & 1 & 0 & 1 & 1 & 1 & 1 & 0 & 0 & 0 \\
\hline 2052412 & 0 & 4 & 1 & 3 & 2 & 3 & 3 & $\overline{1}$ & 4 & 4 & 1 & 1 & 1 & 1 & 1 & 1 & 0 & 0 & 0 \\
\hline 2052413 & 1 & 5 & 1 & 3 & 1 & 4 & 2 & 2 & 4 & 4 & 0 & 0 & 1 & 1 & 1 & 1 & 0 & 0 & 0 \\
\hline 2052414 & 1 & 4 & 1 & 2 & 2 & 2 & 3 & 2 & 4 & 4 & 0 & 0 & 1 & 1 & 3 & 1 & 1 & 0 & 1 \\
\hline 2052415 & 0 & 3 & 1 & 4 & 1 & 5 & 3 & 2 & 4 & 2 & 1 & 1 & 1 & 1 & 1 & 1 & 0 & 0 & 0 \\
\hline 2052416 & 1 & 4 & 1 & 1 & 1 & 2 & 2 & 2 & 4 & 3 & 0 & 0 & 1 & 1 & 1 & 6 & 0 & 1 & 1 \\
\hline 2052417 & 0 & 3 & 1 & 5 & 2 & 5 & 3 & 1 & 4 & 3 & 0 & 0 & 1 & 1 & 1 & 1 & 0 & 0 & 0 \\
\hline
\end{tabular}




\begin{tabular}{|c|c|c|c|c|c|c|c|c|c|c|c|c|c|c|c|c|c|c|c|}
\hline 2052418 & 1 & 3 & 1 & 1 & 1 & 5 & 2 & 2 & 4 & 4 & 0 & 0 & 1 & 1 & 3 & 1 & 1 & 0 & 1 \\
\hline 2052419 & 0 & 1 & 1 & 1 & 1 & 1 & 2 & 2 & 4 & 2 & 0 & 0 & 1 & 1 & 2 & 1 & 1 & 0 & 1 \\
\hline 2052420 & 0 & 2 & 1 & 1 & 1 & 1 & 3 & 2 & 4 & 3 & 1 & 1 & 1 & 1 & 3 & 1 & 1 & 0 & 1 \\
\hline 2052421 & 1 & 5 & 1 & 1 & 1 & 2 & 2 & 2 & 4 & 4 & 0 & 0 & 1 & 1 & 2 & 1 & 1 & 0 & 1 \\
\hline 2052422 & 1 & 1 & 1 & 3 & 2 & 5 & 3 & 2 & 4 & 4 & 0 & 0 & 1 & 1 & 1 & 1 & 0 & 0 & 0 \\
\hline 2052423 & 0 & 1 & 1 & 4 & 2 & 1 & 2 & 1 & 4 & 4 & 0 & 0 & 1 & 1 & 3 & 6 & 1 & 1 & 1 \\
\hline 2052424 & 0 & 2 & 1 & 2 & 2 & 1 & 2 & 1 & 4 & 4 & 0 & 0 & 1 & 1 & 1 & 1 & 0 & 0 & 0 \\
\hline 2052425 & 1 & 3 & 1 & 2 & 1 & 4 & 3 & 2 & 4 & 4 & 0 & 0 & 1 & 1 & 2 & 1 & 1 & 0 & 1 \\
\hline 2052426 & 1 & 3 & 1 & 2 & 1 & 4 & 3 & 2 & 4 & 4 & 0 & 0 & 1 & 1 & 1 & 1 & 0 & 0 & 0 \\
\hline 2052427 & 0 & 2 & 1 & 3 & 1 & 1 & 3 & 1 & 4 & 4 & 1 & 1 & 1 & 1 & 2 & 1 & 1 & 0 & 1 \\
\hline 2052428 & 1 & 3 & 1 & 4 & 2 & 4 & 3 & 1 & 4 & 4 & 1 & 1 & 1 & 1 & 3 & 1 & 1 & 0 & 1 \\
\hline 2052429 & 0 & 4 & 1 & 1 & 1 & 1 & 3 & 2 & 4 & 2 & 0 & 0 & 1 & 1 & 3 & 1 & 1 & 0 & 1 \\
\hline 2052430 & 0 & 5 & 1 & 1 & 2 & 3 & 2 & 1 & 4 & 4 & 0 & 0 & 1 & 1 & 4 & 1 & 1 & 0 & 1 \\
\hline 205251 & 0 & 2 & 1 & 2 & 1 & 2 & 2 & 1 & 2 & 1 & 0 & 0 & 1 & 1 & 1 & 1 & 0 & 0 & 0 \\
\hline 205252 & 0 & 3 & 1 & 1 & 1 & 2 & 1 & 1 & 2 & 1 & 0 & 0 & 1 & 1 & 3 & 1 & 1 & 0 & 1 \\
\hline 205253 & 0 & 2 & 1 & 4 & 1 & 1 & 2 & 2 & 2 & 1 & 0 & 0 & 1 & 1 & 2 & 1 & 1 & 0 & 1 \\
\hline 205254 & 1 & 5 & 1 & 2 & 1 & 2 & 2 & 1 & 2 & 1 & 0 & 0 & 1 & 1 & 3 & 1 & 1 & 0 & 1 \\
\hline 205255 & 0 & 2 & 1 & 1 & 1 & 3 & 1 & 2 & 2 & 1 & 0 & 0 & 1 & 1 & 2 & 1 & 1 & 0 & 1 \\
\hline 205256 & 0 & 2 & 1 & 2 & 1 & 1 & 2 & 2 & 2 & 1 & 0 & 0 & 1 & 1 & 2 & 1 & 1 & 0 & 1 \\
\hline 205257 & 0 & 2 & 1 & 2 & 1 & 2 & 2 & 2 & 2 & 1 & 0 & 0 & 1 & 1 & 3 & 1 & 1 & 0 & 1 \\
\hline 205258 & 1 & 2 & 1 & 1 & 1 & 2 & 1 & 1 & 2 & 1 & 0 & 0 & 1 & 1 & 3 & 1 & 1 & 0 & 1 \\
\hline 205259 & 0 & 3 & 1 & 1 & 2 & 2 & 1 & 2 & 2 & 1 & 0 & 0 & 1 & 1 & 1 & 1 & 0 & 0 & 0 \\
\hline 2052510 & 1 & 5 & 1 & 1 & 1 & 2 & 1 & 1 & 2 & 1 & 0 & 0 & 1 & 1 & 3 & 1 & 1 & 0 & 1 \\
\hline 2052511 & 1 & 2 & 1 & 2 & 1 & 2 & 1 & 2 & 2 & 1 & 0 & 0 & 1 & 1 & 3 & 1 & 1 & 0 & 1 \\
\hline 2052512 & 0 & 1 & 1 & 2 & 1 & 2 & 1 & 2 & 2 & 1 & 0 & 0 & 1 & 1 & 2 & 1 & 1 & 0 & 1 \\
\hline 2052513 & 0 & 2 & 1 & 1 & 1 & 2 & 1 & 2 & 2 & 1 & 0 & 0 & 1 & 1 & 2 & 1 & 1 & 0 & 1 \\
\hline 2052514 & 0 & 2 & 1 & 4 & 1 & 2 & 2 & 2 & 2 & 1 & 0 & 0 & 1 & 1 & 2 & 1 & 1 & 0 & 1 \\
\hline 2052515 & 0 & 2 & 1 & 2 & 1 & 2 & 2 & 2 & 2 & 1 & 1 & 0 & 1 & 1 & 3 & 1 & 1 & 0 & 1 \\
\hline 2052516 & 0 & 2 & 1 & 4 & 1 & 3 & 2 & 2 & 2 & 1 & 0 & 0 & 1 & 1 & 1 & 1 & 0 & 0 & 0 \\
\hline 2052517 & 0 & 2 & 1 & 1 & 1 & 2 & 1 & 1 & 2 & 2 & 0 & 0 & 1 & 1 & 2 & 1 & 1 & 0 & 1 \\
\hline 2052518 & 0 & 2 & 1 & 1 & 1 & 2 & 1 & 2 & 2 & 2 & 0 & 0 & 1 & 1 & 2 & 1 & 1 & 0 & 1 \\
\hline 2052519 & 1 & 2 & 1 & 1 & 1 & 2 & 1 & 2 & 2 & 1 & 0 & 0 & 1 & 1 & 3 & 1 & 1 & 0 & 1 \\
\hline 2052520 & 0 & 1 & 1 & 1 & 1 & 2 & 2 & 2 & 2 & 1 & 0 & 0 & 1 & 1 & 2 & 1 & 1 & 0 & 1 \\
\hline 2052521 & 0 & 3 & 1 & 1 & 1 & 2 & 1 & 2 & 3 & 1 & 0 & 0 & 1 & 1 & 2 & 1 & 1 & 0 & 1 \\
\hline 2052522 & 0 & 2 & 1 & 1 & 1 & 2 & 2 & 2 & 2 & 1 & 0 & 0 & 1 & 1 & 2 & 1 & 1 & 0 & 1 \\
\hline 2052523 & 0 & 3 & 1 & 1 & 1 & 2 & 2 & 2 & 3 & 1 & 0 & 0 & 1 & 1 & 3 & 1 & 1 & 0 & 1 \\
\hline 2052524 & 0 & 3 & 1 & 1 & 1 & 2 & 2 & 2 & 3 & 1 & 0 & 0 & 1 & 1 & 2 & 1 & 1 & 0 & 1 \\
\hline 2052525 & 0 & 3 & 1 & 2 & 1 & 3 & 3 & 2 & 3 & 2 & 0 & 0 & 1 & 1 & 2 & 1 & 1 & 0 & 1 \\
\hline 2052526 & 0 & 2 & 1 & 2 & 1 & 2 & 1 & 2 & 3 & 2 & 0 & 0 & 1 & 1 & 2 & 1 & 1 & 0 & 1 \\
\hline 2052527 & 0 & 1 & 1 & 1 & 1 & 1 & 2 & 1 & 3 & 3 & 0 & 0 & 1 & 1 & 2 & 1 & 1 & 0 & 1 \\
\hline 2052528 & 1 & 3 & 1 & 1 & 1 & 2 & 2 & 2 & 3 & 3 & 0 & 0 & 1 & 1 & 2 & 1 & 1 & 0 & 1 \\
\hline 2052529 & 0 & 3 & 1 & 1 & 1 & 2 & 1 & 2 & 2 & 1 & 0 & 0 & 1 & 1 & 1 & 1 & 0 & 0 & 0 \\
\hline 2052530 & 1 & 3 & 1 & 3 & 1 & 3 & 2 & 2 & 2 & 2 & 0 & 0 & 1 & 1 & 2 & 1 & 1 & 0 & 1 \\
\hline 206261 & 1 & 3 & 1 & 2 & 1 & 2 & 2 & 1 & 3 & 2 & 0 & $\overline{0}$ & 1 & 1 & 1 & 1 & 0 & 0 & 0 \\
\hline 206262 & 1 & 1 & 1 & 2 & 1 & 2 & 1 & 1 & 3 & 2 & 0 & 0 & 1 & 1 & 3 & 1 & 1 & 0 & 1 \\
\hline 206263 & 1 & 3 & 1 & 3 & 1 & 2 & 2 & 2 & 3 & 1 & 0 & 0 & 1 & 1 & 1 & 1 & 0 & 0 & 0 \\
\hline 206264 & 1 & 2 & 1 & 4 & 1 & 2 & 2 & 2 & 3 & 1 & 0 & 0 & 1 & 1 & 1 & 1 & 0 & 0 & 0 \\
\hline 206265 & 1 & 4 & 1 & 2 & 1 & 2 & 2 & 2 & 3 & 1 & 0 & 0 & 1 & 1 & 1 & 1 & 0 & 0 & 0 \\
\hline 206266 & 0 & 1 & 1 & 2 & 1 & 2 & 1 & 1 & 3 & 3 & 0 & $\overline{0}$ & 1 & 1 & 3 & 1 & 1 & 0 & 1 \\
\hline 206267 & 1 & 2 & 1 & 3 & 1 & 2 & 2 & 1 & 3 & 2 & 0 & 0 & 1 & 1 & 1 & 1 & 0 & 0 & 0 \\
\hline 206268 & 0 & 1 & 1 & 4 & 1 & 1 & 2 & 2 & 3 & 3 & 0 & 0 & 1 & 1 & 1 & 1 & 0 & 0 & 0 \\
\hline 206269 & 0 & 4 & 1 & 2 & 1 & 2 & 2 & 2 & 3 & 4 & 0 & 0 & 1 & 1 & 1 & 1 & 0 & 0 & 0 \\
\hline 2062610 & 0 & 1 & 1 & 2 & 1 & 2 & 2 & 2 & 3 & 4 & 0 & 0 & 1 & 1 & 3 & 1 & 1 & 0 & 1 \\
\hline 2062611 & 0 & 2 & 1 & 1 & 1 & 2 & 1 & 1 & 3 & 4 & 0 & $\overline{0}$ & 1 & 1 & 1 & 1 & 0 & 0 & $\overline{0}$ \\
\hline 2062612 & 0 & 1 & 1 & 4 & 1 & 2 & 1 & 2 & 3 & 4 & 0 & 0 & 1 & 1 & 1 & 1 & 0 & 0 & 0 \\
\hline 2062613 & 0 & 3 & 1 & 1 & 1 & 2 & 2 & 2 & 3 & 4 & 0 & 0 & 1 & 1 & 3 & 1 & 1 & 0 & 1 \\
\hline
\end{tabular}




\begin{tabular}{|c|c|c|c|c|c|c|c|c|c|c|c|c|c|c|c|c|c|c|c|}
\hline 2062614 & 0 & 3 & 1 & 2 & 1 & 2 & 2 & 1 & 3 & 4 & 1 & 1 & 1 & 1 & 1 & 1 & 0 & 0 & 0 \\
\hline 2062615 & 1 & 4 & 1 & 1 & 1 & 2 & 1 & 1 & 3 & 3 & 0 & 0 & 1 & 1 & 3 & 1 & 1 & 0 & 1 \\
\hline 2062616 & 1 & 3 & 1 & 1 & 1 & 2 & 1 & 1 & 3 & 4 & 0 & 0 & 1 & 1 & 3 & 1 & 1 & 0 & 1 \\
\hline 2062617 & 0 & 1 & 1 & 3 & 1 & 5 & 1 & 2 & 3 & 3 & 0 & 0 & 1 & 1 & 2 & 1 & 1 & 0 & 1 \\
\hline 2062618 & 1 & 5 & 1 & 1 & 1 & 2 & 2 & 2 & 3 & 3 & 0 & 0 & 1 & 1 & 2 & 1 & 1 & 0 & 1 \\
\hline 2062619 & 0 & 2 & 1 & 2 & 1 & 2 & 2 & 2 & 3 & 4 & 0 & 0 & 1 & 1 & 3 & 1 & 1 & 0 & 1 \\
\hline 2062620 & 0 & 2 & 1 & 1 & 1 & 2 & 2 & 2 & 3 & 2 & 0 & 0 & 1 & 1 & 2 & 1 & 1 & 0 & 1 \\
\hline 2062621 & 0 & 2 & 1 & 1 & 1 & 1 & 1 & 2 & 3 & 2 & 0 & 0 & 1 & 1 & 1 & 1 & 0 & 0 & 0 \\
\hline 2062622 & 0 & 2 & 1 & 1 & 1 & 3 & 3 & 2 & 3 & 1 & 0 & 0 & 1 & 1 & 1 & 1 & 0 & 0 & 0 \\
\hline 2062623 & 0 & 4 & 1 & 1 & 1 & 2 & 2 & 1 & 3 & 2 & 0 & 0 & 1 & 1 & 3 & 1 & 1 & 0 & 1 \\
\hline 2062624 & 1 & 3 & 1 & 2 & 1 & 2 & 2 & 2 & 3 & 1 & 0 & 0 & 1 & 1 & 1 & 1 & 0 & 0 & 0 \\
\hline 2062625 & 1 & 3 & 1 & 3 & 1 & 2 & 2 & 1 & 3 & 2 & 0 & 0 & 1 & 1 & 3 & 1 & 1 & 0 & 1 \\
\hline 206271 & 0 & 4 & 1 & 1 & 1 & 2 & 1 & 2 & 3 & 1 & 0 & 0 & 1 & 1 & 2 & 1 & 1 & 0 & 1 \\
\hline 206272 & 0 & 1 & 1 & 4 & 1 & 2 & 3 & 1 & 3 & 1 & 0 & 0 & 1 & 1 & 2 & 1 & 1 & 0 & 1 \\
\hline 206273 & 0 & 2 & 1 & 2 & 1 & 2 & 2 & 2 & 3 & 1 & 0 & 0 & 1 & 1 & 2 & 1 & 1 & 0 & 1 \\
\hline 206274 & 0 & 1 & 1 & 2 & 1 & 1 & 3 & 1 & 3 & 1 & 0 & 0 & 1 & 1 & 2 & 1 & 1 & 0 & 1 \\
\hline 206275 & 1 & 1 & 1 & 1 & 1 & 2 & 1 & 2 & 3 & 1 & 0 & 0 & 1 & 1 & 2 & 1 & 1 & 0 & 1 \\
\hline 206276 & 0 & 3 & 1 & 2 & 1 & 2 & 2 & 2 & 3 & 4 & 0 & 0 & 1 & 1 & 2 & 1 & 1 & 0 & 1 \\
\hline 206277 & 1 & 1 & 1 & 4 & 1 & 2 & 2 & 1 & 3 & 4 & 0 & 0 & 1 & 1 & 1 & 1 & 0 & 0 & 0 \\
\hline 206278 & 0 & 2 & 1 & 4 & 1 & 2 & 2 & 1 & 3 & 4 & 0 & 0 & 1 & 1 & 2 & 1 & 1 & 0 & 1 \\
\hline 206279 & 1 & 3 & 1 & 2 & 1 & 2 & 1 & 2 & 3 & 4 & 0 & 0 & 1 & 1 & 1 & 1 & 0 & 0 & 0 \\
\hline 2062710 & 0 & 2 & 1 & 2 & 1 & 2 & 1 & 1 & 3 & 4 & 0 & 0 & 1 & 1 & 1 & 1 & 0 & 0 & 0 \\
\hline 2062711 & 0 & 3 & 1 & 1 & 1 & 2 & 1 & 2 & 1 & 2 & 0 & 0 & 1 & 1 & 2 & 1 & 1 & 0 & 1 \\
\hline 2062712 & 0 & 2 & 1 & 1 & 1 & 2 & 2 & 1 & 3 & 4 & 0 & 0 & 1 & 1 & 1 & 1 & 0 & 0 & 0 \\
\hline 2062713 & 1 & 1 & 1 & 3 & 1 & 2 & 2 & 2 & 3 & 4 & 0 & 0 & 1 & 1 & 1 & 1 & 0 & 0 & 0 \\
\hline 2062714 & 1 & 1 & 1 & 2 & 1 & 2 & 2 & 1 & 3 & 4 & 0 & 0 & 1 & 1 & 2 & 1 & 1 & 0 & 1 \\
\hline 2062715 & 1 & 1 & 1 & 1 & 1 & 2 & 2 & 2 & 3 & 4 & 0 & 0 & 1 & 1 & 1 & 1 & 0 & 0 & 0 \\
\hline 2062716 & 1 & 2 & 1 & 3 & 1 & 2 & 1 & 2 & 3 & 4 & 0 & 0 & 1 & 1 & 1 & 1 & 0 & 0 & 0 \\
\hline 2062717 & 0 & 1 & 1 & 3 & 1 & 2 & 2 & 1 & 3 & 4 & 0 & 0 & 1 & 1 & 2 & 1 & 1 & 0 & 1 \\
\hline 2062718 & 1 & 1 & 1 & 1 & 1 & 2 & 1 & 1 & 3 & 4 & 0 & 0 & 1 & 1 & 2 & 1 & 1 & 0 & 1 \\
\hline 2062719 & 0 & 2 & 1 & 2 & 1 & 2 & 2 & 1 & 3 & 4 & 0 & 0 & 1 & 1 & 2 & 1 & 1 & 0 & 1 \\
\hline 2062720 & 0 & 1 & 1 & 3 & 1 & 1 & 2 & 1 & 3 & 4 & 0 & 0 & 1 & 1 & 2 & 1 & 1 & 0 & 1 \\
\hline 2062721 & 1 & 4 & 1 & 1 & 1 & 2 & 1 & 2 & 3 & 4 & 0 & 0 & 1 & 1 & 2 & 1 & 1 & 0 & 1 \\
\hline 2062722 & 0 & 2 & 1 & 3 & 1 & 2 & 1 & 2 & 3 & 4 & 0 & 0 & 1 & 1 & 1 & 1 & 0 & 0 & 0 \\
\hline 2062723 & 1 & 3 & 1 & 2 & 1 & 2 & 1 & 2 & 3 & 4 & 0 & 0 & 1 & 1 & 1 & 1 & 0 & 0 & 0 \\
\hline 2062724 & 0 & 2 & 1 & 2 & 1 & 2 & 2 & 2 & 3 & 4 & 0 & 0 & 1 & 1 & 1 & 1 & 0 & 0 & 0 \\
\hline 2062725 & 1 & 2 & 1 & 2 & 1 & 2 & 2 & 2 & 3 & 4 & 0 & 0 & 1 & 1 & 1 & 1 & 0 & 0 & 0 \\
\hline 2062726 & 1 & 1 & 1 & 1 & 1 & 2 & 1 & 1 & 1 & 2 & 0 & 0 & 1 & 1 & 4 & 5 & 1 & 1 & 1 \\
\hline 2062727 & 1 & 3 & 1 & 1 & 1 & 2 & 2 & 2 & 3 & 2 & 0 & 0 & 1 & 1 & 3 & 6 & 1 & 1 & 1 \\
\hline 2062728 & 0 & 1 & 1 & 2 & 1 & 2 & 2 & 2 & 3 & 2 & 0 & 0 & 1 & 1 & 2 & 1 & 1 & 0 & 1 \\
\hline 2062729 & 0 & 3 & 1 & 1 & 1 & 2 & 2 & 2 & 3 & 2 & 0 & 0 & 1 & 1 & 2 & 1 & 1 & 0 & 1 \\
\hline 2062730 & 0 & 2 & 1 & 2 & 1 & 1 & 1 & 2 & 3 & 2 & 0 & 0 & 1 & 1 & 2 & 1 & 1 & 0 & 1 \\
\hline 206281 & 0 & 3 & 1 & 3 & 1 & 1 & 2 & 2 & 3 & 3 & 0 & 0 & 1 & 1 & 2 & 2 & 1 & 0 & 1 \\
\hline 206282 & 1 & 2 & 1 & 4 & 1 & 2 & 2 & 2 & 3 & 2 & 0 & 0 & 1 & 1 & 5 & 5 & 1 & 1 & 1 \\
\hline 206283 & 1 & 3 & 1 & 2 & 1 & 2 & 2 & 2 & 3 & 1 & 0 & 0 & 1 & 1 & 1 & 1 & 0 & 0 & 0 \\
\hline 206284 & 1 & 3 & 1 & 2 & 1 & 2 & 2 & 2 & 3 & 1 & 0 & 0 & 1 & 1 & 2 & 1 & 1 & 0 & 1 \\
\hline 206285 & 0 & 2 & 1 & 2 & 1 & 3 & 2 & 2 & 3 & 1 & \begin{tabular}{l|l}
0 \\
\end{tabular} & 0 & 1 & 1 & 3 & 1 & 1 & 0 & 1 \\
\hline 206286 & 0 & 5 & 1 & 2 & 1 & 1 & 2 & 2 & 3 & 1 & 0 & 0 & 1 & 1 & 2 & 1 & 1 & 0 & 1 \\
\hline 206287 & 0 & 2 & 1 & 2 & 1 & 3 & 2 & 2 & 3 & 1 & 0 & 0 & 1 & 1 & 2 & 1 & 1 & 0 & 1 \\
\hline 206288 & 0 & 2 & 1 & 1 & 1 & 1 & 2 & 2 & 2 & 4 & 0 & 0 & 1 & 1 & 2 & 1 & 1 & 0 & 1 \\
\hline 206289 & 0 & 1 & 1 & 4 & 1 & 1 & 2 & 2 & 2 & 4 & 0 & 0 & 1 & 1 & 4 & 1 & 1 & 0 & 1 \\
\hline 2062810 & 1 & 2 & 1 & 1 & 1 & 2 & 2 & 2 & 2 & 4 & 0 & 0 & 1 & 1 & 2 & 1 & 1 & 0 & 1 \\
\hline 2062811 & 1 & 4 & 1 & 1 & 1 & 2 & 1 & 2 & 2 & 4 & 0 & 0 & 1 & 0 & 5 & 5 & 1 & 1 & 1 \\
\hline 2062812 & 1 & 2 & 1 & 1 & 1 & 2 & 1 & 2 & 2 & 4 & 0 & 0 & 1 & 1 & 4 & 2 & 1 & 0 & 1 \\
\hline 2062813 & 0 & 1 & 1 & 3 & 1 & 3 & 1 & 1 & 2 & 4 & 0 & 0 & 1 & 1 & 2 & 1 & 1 & 0 & 1 \\
\hline 2062814 & 1 & 4 & 1 & 4 & 2 & 2 & 2 & 2 & 1 & 1 & 0 & 0 & 1 & 1 & 4 & 2 & 1 & 0 & 1 \\
\hline
\end{tabular}




\begin{tabular}{|c|c|c|c|c|c|c|c|c|c|c|c|c|c|c|c|c|c|c|c|}
\hline 2062815 & 0 & 3 & 1 & 2 & 1 & 1 & 1 & 2 & 1 & 3 & 0 & 0 & 1 & 1 & 5 & 5 & 1 & 1 & 1 \\
\hline 2062816 & 1 & 1 & 1 & 2 & 2 & 2 & 2 & 1 & 1 & 2 & 0 & 0 & 1 & 1 & 2 & 2 & 1 & 0 & 1 \\
\hline 2062817 & 0 & 4 & 1 & 2 & 1 & 1 & 2 & 2 & 1 & 1 & 0 & 0 & 1 & 1 & 1 & 1 & 0 & 0 & 0 \\
\hline 2062818 & 0 & 3 & 1 & 1 & 1 & 1 & 2 & 2 & 2 & 4 & 0 & 0 & 1 & 1 & 4 & 1 & 1 & 0 & 1 \\
\hline 2062819 & 0 & 4 & 1 & 2 & 2 & 5 & 2 & 2 & 1 & 2 & 0 & 0 & 1 & 1 & 2 & 1 & 1 & 0 & 1 \\
\hline 2062820 & 1 & 4 & 1 & 1 & 2 & 2 & 3 & 2 & 1 & 4 & 0 & 0 & 1 & 0 & 5 & 5 & 1 & 1 & 1 \\
\hline 2062821 & 0 & 3 & 1 & 1 & 2 & 2 & 2 & 2 & 2 & 1 & 0 & 0 & 1 & 1 & 3 & 2 & 1 & 0 & 1 \\
\hline 2062822 & 1 & 3 & 1 & 2 & 2 & 2 & 3 & 2 & 1 & 2 & 0 & 0 & 1 & 1 & 1 & 6 & 0 & 1 & 1 \\
\hline 2062823 & 1 & 3 & 1 & 1 & 1 & 2 & 3 & 2 & 1 & 1 & 0 & 0 & 1 & 1 & 2 & 1 & 1 & 0 & 1 \\
\hline 2062824 & 0 & 3 & 1 & 1 & 2 & 2 & 2 & 2 & 1 & 1 & 0 & 0 & 1 & 1 & 1 & 1 & 0 & 0 & 0 \\
\hline 2062825 & 0 & 2 & 1 & 2 & 2 & 1 & 2 & 2 & 1 & 1 & 0 & 0 & 1 & 1 & 1 & 1 & 0 & 0 & 0 \\
\hline 2062826 & 1 & 3 & 1 & 1 & 2 & 2 & 1 & 1 & 1 & 1 & 0 & 0 & 1 & 1 & 3 & 5 & 1 & 1 & 1 \\
\hline 2062827 & 1 & 3 & 1 & 4 & 1 & 3 & 3 & 2 & 1 & 2 & 0 & 0 & 1 & 1 & 5 & 7 & 1 & 1 & 1 \\
\hline 2062828 & 1 & 5 & 1 & 2 & 1 & 2 & 2 & 1 & 3 & 3 & 0 & 0 & 1 & 1 & 2 & 2 & 1 & 0 & 1 \\
\hline 2062829 & 1 & 5 & 1 & 1 & 1 & 2 & 2 & 2 & 3 & 1 & 0 & 0 & 1 & 1 & 3 & 1 & 1 & 0 & 1 \\
\hline 2062830 & 1 & 4 & 1 & 4 & 2 & 2 & 3 & 2 & 1 & 1 & 0 & 0 & 1 & 1 & 1 & 1 & 0 & 0 & 0 \\
\hline 206291 & 1 & 5 & 1 & 1 & 1 & 2 & 2 & 1 & 1 & 1 & 0 & 0 & 1 & 1 & 2 & 1 & 1 & 0 & 1 \\
\hline 206292 & 0 & 3 & 1 & 2 & 1 & 2 & 2 & 2 & 1 & 1 & 0 & 0 & 1 & 1 & 1 & 1 & 0 & 0 & 0 \\
\hline 206293 & 0 & 3 & 1 & 1 & 1 & 3 & 2 & 1 & 1 & 2 & 0 & 0 & 1 & 1 & 1 & 1 & 0 & 0 & 0 \\
\hline 206294 & 1 & 2 & 1 & 2 & 1 & 2 & 2 & 2 & 1 & 3 & 0 & 0 & 1 & 1 & 2 & 1 & 1 & 0 & 1 \\
\hline 206295 & 1 & 5 & 1 & 1 & 1 & 2 & 2 & 2 & 1 & 2 & 1 & 0 & 1 & 1 & 2 & 1 & 1 & 0 & 1 \\
\hline 206296 & 0 & 4 & 1 & 1 & 1 & 2 & 2 & 2 & 1 & 3 & 0 & 0 & 1 & 1 & 4 & 1 & 1 & 0 & 1 \\
\hline 206297 & 1 & 5 & 1 & 1 & 1 & 2 & 2 & 2 & 1 & 4 & 0 & 0 & 1 & 1 & 2 & 1 & 1 & 0 & 1 \\
\hline 206298 & 1 & 5 & 1 & 1 & 1 & 2 & 1 & 1 & 1 & 4 & 0 & 0 & 0 & 99 & 99 & 99 & 99 & 99 & 99 \\
\hline 206299 & 0 & 4 & 1 & 2 & 1 & 2 & 3 & 2 & 1 & 4 & 0 & 0 & 1 & 1 & 1 & 1 & 0 & 0 & 0 \\
\hline 2062910 & 1 & 5 & 1 & 1 & 1 & 2 & 3 & 1 & 1 & 4 & 0 & 0 & 0 & 99 & 99 & 99 & 99 & 99 & 99 \\
\hline 2062911 & 0 & 2 & 1 & 2 & 2 & 1 & 2 & 2 & 1 & 4 & 1 & 1 & 1 & 1 & 2 & 1 & 1 & 0 & 1 \\
\hline 2062912 & 0 & 3 & 1 & 1 & 2 & 2 & 2 & 2 & 1 & 4 & 0 & 0 & 1 & 1 & 2 & 1 & 1 & 0 & 1 \\
\hline 2062913 & 0 & 2 & 1 & 1 & 1 & 2 & 2 & 2 & 1 & 1 & 0 & 0 & 1 & 1 & 1 & 1 & 0 & 0 & 0 \\
\hline 2062914 & 0 & 3 & 1 & 1 & 1 & 2 & 2 & 2 & 1 & 1 & 0 & 0 & 1 & 1 & 2 & 1 & 1 & 0 & 1 \\
\hline 2062915 & 1 & 1 & 1 & 5 & 1 & 4 & 2 & 2 & 1 & 1 & 1 & 1 & 1 & 1 & 1 & 1 & 0 & 0 & 0 \\
\hline 2062916 & 1 & 2 & 1 & 4 & 1 & 5 & 3 & 2 & 1 & 1 & 1 & 1 & 1 & 1 & 2 & 1 & 1 & 0 & 1 \\
\hline 2062917 & 1 & 5 & 1 & 1 & 1 & 2 & 2 & 2 & 1 & 3 & 0 & 0 & 1 & 1 & 1 & 1 & 0 & 0 & 0 \\
\hline 2062918 & 0 & 2 & 1 & 3 & 1 & 1 & 2 & 2 & 1 & 2 & 0 & 0 & 1 & 1 & 2 & 1 & 1 & 0 & 1 \\
\hline 2062919 & 0 & 3 & 1 & 1 & 1 & 1 & 2 & 2 & 1 & 1 & 0 & 0 & 1 & 1 & 2 & 4 & 1 & 1 & 1 \\
\hline 2062920 & 0 & 1 & 1 & 4 & 1 & 3 & 3 & 2 & 1 & 1 & 0 & 0 & 1 & 1 & 2 & 6 & 1 & 1 & 1 \\
\hline 2062921 & 1 & 3 & 1 & 2 & 1 & 2 & 3 & 2 & 1 & 1 & 0 & 0 & 1 & 1 & 1 & 1 & 0 & 0 & 0 \\
\hline 2062922 & 0 & 2 & 1 & 1 & 1 & 1 & 2 & 2 & 1 & 2 & 0 & 0 & 1 & 1 & 3 & 1 & 1 & 0 & 1 \\
\hline 2062923 & 0 & 3 & 1 & 4 & 1 & 1 & 2 & 2 & 1 & 3 & 0 & 0 & 1 & 1 & 2 & 1 & 1 & 0 & 1 \\
\hline 2062924 & 1 & 4 & 1 & 1 & 1 & 2 & 3 & 2 & 1 & 1 & 0 & 0 & 1 & 1 & 3 & 6 & 1 & 1 & 1 \\
\hline 2062925 & 1 & 2 & 1 & 1 & 1 & 2 & 2 & 2 & 1 & 1 & 0 & 0 & 1 & 1 & 2 & 1 & 1 & 0 & 1 \\
\hline 2062926 & 0 & 2 & 1 & 2 & 1 & 1 & 2 & 2 & 1 & 1 & 0 & 0 & 1 & 1 & 2 & 5 & 1 & 1 & 1 \\
\hline 2062927 & 0 & 2 & 1 & 2 & 1 & 1 & 2 & 2 & 1 & 1 & 0 & 0 & 1 & 1 & 1 & 2 & 0 & 0 & 0 \\
\hline 2062928 & 0 & 2 & 1 & 1 & 1 & 1 & 2 & 2 & 1 & 2 & 0 & 0 & 1 & 1 & 1 & 1 & 0 & 0 & 0 \\
\hline 2062929 & 0 & 2 & 1 & 1 & 1 & 1 & 2 & 2 & 1 & 3 & 0 & 0 & 1 & 1 & 2 & 1 & 1 & 0 & 1 \\
\hline 2062930 & 1 & 4 & 1 & 2 & 1 & 2 & 2 & 2 & 1 & 3 & 0 & 0 & 1 & 1 & 2 & 2 & 1 & 0 & 1 \\
\hline 206301 & 0 & 5 & 1 & 2 & 1 & 3 & 2 & 1 & 1 & 1 & 0 & 0 & 1 & 1 & 1 & 1 & 0 & 0 & 0 \\
\hline 206302 & 0 & 4 & 1 & 1 & 1 & 3 & 2 & 1 & 1 & 2 & 0 & 0 & 1 & 1 & 1 & 1 & 0 & 0 & 0 \\
\hline 206303 & 0 & 5 & 1 & 4 & 2 & 1 & 1 & 1 & 1 & 1 & 0 & 0 & 0 & 99 & 99 & 99 & 99 & 99 & 99 \\
\hline 206304 & 0 & 1 & 1 & 4 & 2 & 1 & 2 & 1 & 1 & 4 & 1 & 1 & 1 & 1 & 1 & 1 & o & 0 & 0 \\
\hline 206305 & 0 & 5 & 1 & 1 & 1 & 3 & 2 & 1 & 1 & 2 & 0 & 0 & 0 & 99 & 99 & 99 & 99 & 99 & 99 \\
\hline 206306 & 1 & 4 & 1 & 3 & 1 & 2 & 2 & 2 & 1 & 1 & 0 & 0 & 1 & 1 & 1 & 1 & 0 & 0 & 0 \\
\hline 206307 & 1 & 3 & 1 & 3 & 1 & 4 & 2 & 1 & 1 & 1 & 0 & 0 & 1 & 1 & 1 & 1 & 0 & 0 & 0 \\
\hline 206308 & 0 & 5 & 1 & 1 & 1 & 1 & 2 & 1 & 1 & 3 & 0 & 0 & 0 & 99 & 99 & 99 & 99 & 99 & 99 \\
\hline 206309 & 0 & 2 & 1 & 5 & 1 & 5 & 2 & 1 & 1 & 1 & 1 & 1 & 1 & 1 & 1 & 1 & 0 & 0 & 0 \\
\hline 2063010 & 1 & 3 & 1 & 1 & 2 & 4 & 3 & 1 & 1 & 3 & 1 & 0 & 1 & 1 & 1 & 1 & 0 & 0 & \\
\hline
\end{tabular}




\begin{tabular}{|c|c|c|c|c|c|c|c|c|c|c|c|c|c|c|c|c|c|c|c|}
\hline 2063011 & 1 & 4 & 1 & 2 & 1 & 2 & 3 & 1 & 1 & 1 & 1 & 1 & 1 & 1 & 1 & 1 & 0 & 0 & 0 \\
\hline 2063012 & 0 & 2 & 1 & 2 & 2 & 1 & 2 & 1 & 1 & 1 & 0 & 0 & 1 & 1 & 1 & 1 & 0 & 0 & 0 \\
\hline 2063013 & 0 & 1 & 1 & 5 & 2 & 1 & 3 & 1 & 1 & 4 & 0 & 0 & 1 & 1 & 1 & 1 & 0 & 0 & 0 \\
\hline 2063014 & 0 & 5 & 1 & 4 & 2 & 3 & 3 & 1 & 1 & 2 & 1 & 1 & 1 & 1 & 1 & 1 & 0 & 0 & 0 \\
\hline 2063015 & 1 & 5 & 1 & 1 & 2 & 2 & 2 & 1 & 1 & 3 & 0 & 0 & 0 & 99 & 99 & 99 & 99 & 99 & 99 \\
\hline 2063016 & 0 & 3 & 1 & 2 & 1 & 1 & 2 & 1 & 1 & 4 & 0 & 0 & 1 & 1 & 1 & 1 & 0 & 0 & 0 \\
\hline 2063017 & 1 & 4 & 1 & 3 & 1 & 4 & 3 & 1 & 1 & 4 & 1 & 1 & 1 & 1 & 1 & 1 & 0 & 0 & 0 \\
\hline 2063018 & 0 & 3 & 1 & 4 & 1 & 1 & 2 & 1 & 1 & 3 & 1 & 0 & 1 & 1 & 1 & 1 & 0 & 0 & 0 \\
\hline 2063019 & 0 & 3 & 1 & 2 & 2 & 1 & 1 & 1 & 1 & 1 & 0 & 0 & 1 & 1 & 1 & 5 & 0 & 1 & 1 \\
\hline 2063020 & 0 & 2 & 1 & 2 & 2 & 3 & 3 & 1 & 1 & 1 & 1 & 0 & 1 & 1 & 1 & 1 & 0 & 0 & 0 \\
\hline 2063021 & 0 & 1 & 1 & 2 & 1 & 3 & 2 & 1 & 1 & 4 & 0 & 0 & 1 & 1 & 1 & 1 & 0 & 0 & 0 \\
\hline 2063022 & 1 & 2 & 1 & 2 & 1 & 2 & 2 & 1 & 1 & 1 & 0 & 0 & 1 & 1 & 1 & 1 & 0 & 0 & 0 \\
\hline 2063023 & 1 & 2 & 1 & 2 & 1 & 2 & 2 & 1 & 1 & 1 & 0 & 0 & 1 & 1 & 1 & 1 & 0 & 0 & 0 \\
\hline 2063024 & 1 & 3 & 1 & 2 & 1 & 2 & 2 & 1 & 1 & 3 & 0 & 0 & 1 & 1 & 1 & 5 & 0 & 1 & 1 \\
\hline 2063025 & 0 & 5 & 1 & 2 & 1 & 2 & 2 & 1 & 1 & 1 & 0 & 0 & 0 & 99 & 99 & 99 & 99 & 99 & 99 \\
\hline 206311 & 0 & 1 & 1 & 2 & 1 & 1 & 2 & 1 & 4 & 4 & 0 & 0 & 1 & 1 & 1 & 1 & 0 & 0 & 0 \\
\hline 206312 & 0 & 2 & 1 & 3 & 1 & 1 & 2 & 1 & 4 & 4 & 0 & 0 & 1 & 1 & 1 & 1 & 0 & 0 & 0 \\
\hline 206313 & 1 & 2 & 1 & 2 & 1 & 2 & 2 & 2 & 4 & 3 & 0 & 0 & 0 & 99 & 99 & 99 & 99 & 99 & 99 \\
\hline 206314 & 1 & 3 & 1 & 1 & 1 & 2 & 2 & 1 & 4 & 4 & 0 & 0 & 1 & 1 & 1 & 1 & 0 & 0 & 0 \\
\hline 206315 & 0 & 2 & 1 & 2 & 1 & 1 & 2 & 1 & 4 & 2 & 1 & 0 & 1 & 1 & 1 & 1 & 0 & 0 & 0 \\
\hline 206316 & 0 & 1 & 1 & 5 & 1 & 1 & 3 & 1 & 4 & 4 & 0 & 0 & 1 & 1 & 1 & 1 & 0 & 0 & 0 \\
\hline 206317 & 0 & 2 & 1 & 1 & 1 & 3 & 2 & 1 & 4 & 4 & 1 & 0 & 1 & 1 & 1 & 1 & 0 & 0 & 0 \\
\hline 206318 & 1 & 3 & 1 & 1 & 1 & 2 & 2 & 1 & 4 & 1 & 1 & 0 & 1 & 1 & 1 & 1 & 0 & 0 & 0 \\
\hline 206319 & 0 & 5 & 1 & 1 & 1 & 1 & 1 & 1 & 4 & 1 & 0 & 0 & 0 & 99 & 99 & 99 & 99 & 99 & 99 \\
\hline 2063110 & 1 & 2 & 1 & 2 & 1 & 2 & 2 & 1 & 4 & 3 & 1 & 0 & 1 & 1 & 1 & 1 & 0 & 0 & 0 \\
\hline 2063111 & 0 & 3 & 1 & 1 & 1 & 3 & 2 & 1 & 4 & 4 & 0 & 0 & 1 & 1 & 1 & 1 & 0 & 0 & 0 \\
\hline 2063112 & 0 & 2 & 1 & 1 & 1 & 1 & 2 & 1 & 4 & 3 & 0 & 0 & 1 & 1 & 1 & 1 & 0 & 0 & 0 \\
\hline 2063113 & 1 & 2 & 1 & 2 & 1 & 2 & 2 & 1 & 4 & 2 & 0 & 0 & 1 & 1 & 1 & 1 & 0 & 0 & 0 \\
\hline 2063114 & 1 & 3 & 1 & 2 & 1 & 2 & 2 & 1 & 4 & 2 & 1 & 0 & 1 & 1 & 1 & 1 & 0 & 0 & 0 \\
\hline 2063115 & 0 & 3 & 1 & 2 & 1 & 1 & 2 & 1 & 4 & 4 & 0 & 0 & 0 & 99 & 99 & 99 & 99 & 99 & 99 \\
\hline 2063116 & 1 & 2 & 1 & 2 & 1 & 2 & 2 & 1 & 4 & 3 & 1 & 0 & 1 & 1 & 1 & 1 & 0 & 0 & 0 \\
\hline 2063117 & 0 & 3 & 1 & 3 & 2 & 1 & 2 & 1 & 4 & 4 & 0 & 0 & 1 & 1 & 1 & 1 & 0 & 0 & 0 \\
\hline 2063118 & 1 & 3 & 1 & 2 & 1 & 3 & 2 & 1 & 4 & 3 & 0 & 0 & 1 & 1 & 4 & 1 & 1 & 0 & 1 \\
\hline 2063119 & 0 & 2 & 1 & 2 & 1 & 1 & 2 & 1 & 4 & 3 & 0 & 0 & 1 & 1 & 1 & 1 & 0 & 0 & 0 \\
\hline 2063120 & 1 & 1 & 1 & 2 & 1 & 2 & 2 & 1 & 4 & 1 & 0 & 0 & 1 & 1 & 1 & 1 & 0 & 0 & 0 \\
\hline 2063121 & 1 & 2 & 1 & 2 & 1 & 2 & 2 & 1 & 4 & 4 & 0 & 0 & 1 & 1 & 1 & 1 & 0 & 0 & 0 \\
\hline 2063122 & 1 & 4 & 1 & 1 & 1 & 2 & 2 & 1 & 4 & 3 & 0 & 0 & 1 & 1 & 1 & 1 & 0 & 0 & 0 \\
\hline 2063123 & 0 & 2 & 1 & 1 & 1 & 1 & 2 & 1 & 4 & 3 & 0 & 0 & 1 & 1 & 1 & 1 & 0 & 0 & 0 \\
\hline 2063124 & 0 & 1 & 1 & 1 & 1 & 1 & 3 & 1 & 4 & 1 & 0 & 0 & 1 & 1 & 1 & 1 & 0 & 0 & 0 \\
\hline 2063125 & 1 & 2 & 1 & 2 & 1 & 2 & 3 & 2 & 4 & 4 & 0 & 0 & 1 & 1 & 1 & 1 & 0 & 0 & 0 \\
\hline 206321 & 0 & 2 & 1 & 1 & 1 & 1 & 1 & 2 & 4 & 4 & 0 & 0 & 1 & 1 & 2 & 1 & 1 & 0 & 1 \\
\hline 206322 & 0 & 2 & 1 & 1 & 1 & 3 & 1 & 1 & 4 & 4 & 0 & 0 & 1 & 1 & 4 & 1 & 1 & 0 & 1 \\
\hline 206323 & 0 & 1 & 1 & 2 & 1 & 1 & 2 & 1 & 4 & 4 & 0 & 0 & 1 & 1 & 3 & 1 & 1 & 0 & 1 \\
\hline 206324 & 1 & 4 & 1 & 2 & 1 & 2 & 1 & 1 & 4 & 4 & 0 & 0 & 1 & 1 & 2 & 1 & 1 & 0 & 1 \\
\hline 206325 & 0 & 1 & 1 & 3 & 1 & 1 & 2 & 1 & 4 & 4 & 0 & 0 & 1 & 1 & 3 & 1 & 1 & 0 & 1 \\
\hline 206326 & 0 & 2 & 1 & 1 & 1 & 2 & 1 & 2 & 4 & 4 & 0 & 0 & 1 & 1 & 3 & 1 & 1 & 0 & 1 \\
\hline 206327 & 0 & 5 & 1 & 1 & 1 & 2 & 1 & 2 & 4 & 4 & 0 & 0 & 1 & 1 & 3 & 1 & 1 & 0 & 1 \\
\hline 206328 & 0 & 3 & 1 & 1 & 1 & 1 & 1 & 2 & 4 & 4 & 0 & 0 & 1 & 1 & 3 & 2 & 1 & 0 & 1 \\
\hline 206329 & 0 & 1 & 1 & 3 & 1 & 1 & 3 & 2 & 1 & 1 & 0 & 0 & 1 & 1 & 3 & 1 & 1 & 0 & 1 \\
\hline 2063210 & 0 & 1 & 1 & 3 & 1 & 3 & 1 & 2 & 1 & 1 & 0 & 0 & 1 & 1 & 2 & 1 & 1 & 0 & 1 \\
\hline 2063211 & 1 & 4 & 1 & 1 & 1 & 2 & 1 & 2 & 1 & 1 & 0 & 0 & 1 & 1 & 3 & 1 & 1 & 0 & 1 \\
\hline 2063212 & 1 & 3 & 1 & 2 & 1 & 2 & 2 & 2 & 1 & 1 & 1 & 1 & 1 & 1 & 3 & 1 & 1 & 0 & 1 \\
\hline 2063213 & 0 & 3 & 1 & 1 & 1 & 2 & 2 & 2 & 1 & 1 & 0 & 0 & 1 & 1 & 2 & 1 & 1 & 0 & 1 \\
\hline 2063214 & 0 & 2 & 1 & 2 & 2 & 1 & 2 & 2 & 4 & 4 & 0 & 0 & 1 & 1 & 2 & 1 & 1 & 0 & 1 \\
\hline 2063215 & 0 & 1 & 1 & 3 & 1 & 5 & 2 & 2 & 4 & 4 & 0 & 0 & 1 & 1 & 2 & 1 & 1 & 0 & 1 \\
\hline 2063216 & 0 & 4 & 1 & 1 & 1 & 3 & 2 & 1 & 4 & 4 & 0 & 0 & 1 & 1 & 2 & 1 & 1 & 0 & \\
\hline
\end{tabular}




\begin{tabular}{|c|c|c|c|c|c|c|c|c|c|c|c|c|c|c|c|c|c|c|c|}
\hline 2063217 & 1 & 2 & 1 & 2 & 1 & 2 & 2 & 2 & 1 & 2 & 0 & 0 & 1 & 1 & 1 & 1 & 0 & 0 & 0 \\
\hline 2063218 & 1 & 1 & 1 & 4 & 1 & 2 & 2 & 2 & 1 & 2 & 0 & 0 & 1 & 1 & 2 & 1 & 1 & 0 & 1 \\
\hline 2063219 & 0 & 5 & 1 & 2 & 1 & 2 & 2 & 2 & 1 & 1 & 0 & 0 & 1 & 1 & 3 & 1 & 1 & 0 & 1 \\
\hline 2063220 & 0 & 2 & 1 & 1 & 1 & 1 & 2 & 2 & 1 & 1 & 0 & 0 & 1 & 1 & 3 & 1 & 1 & 0 & 1 \\
\hline 2063221 & 1 & 2 & 1 & 2 & 1 & 2 & 2 & 1 & 1 & 4 & 0 & 0 & 1 & 1 & 2 & 1 & 1 & 0 & 1 \\
\hline 2063222 & 0 & 3 & 1 & 2 & 1 & 1 & 3 & 2 & 1 & 1 & 0 & 0 & 1 & 1 & 3 & 1 & 1 & 0 & 1 \\
\hline 2063223 & 0 & 2 & 1 & 2 & 1 & 1 & 2 & 2 & 4 & 1 & 0 & 0 & 1 & 1 & 4 & 1 & 1 & \begin{tabular}{l|l}
0 \\
\end{tabular} & 1 \\
\hline 2063224 & 0 & 5 & 1 & 1 & 1 & 3 & 2 & 1 & 1 & 1 & 0 & 0 & 1 & 1 & 4 & 1 & 1 & 0 & 1 \\
\hline 2063225 & 0 & 3 & 1 & 1 & 1 & 3 & 1 & 2 & 4 & 4 & 0 & 0 & 1 & 1 & 2 & 4 & 1 & 1 & 1 \\
\hline 2063226 & 1 & 2 & 1 & 2 & 1 & 2 & 1 & 1 & 1 & 2 & 0 & 0 & 1 & 1 & 2 & 1 & 1 & 0 & 1 \\
\hline 2063227 & 1 & 3 & 1 & 3 & 1 & 2 & 2 & 2 & 1 & 2 & 0 & 0 & 1 & 1 & 2 & 1 & 1 & 0 & 1 \\
\hline 2063228 & 1 & 1 & 1 & 3 & 1 & 2 & 2 & 2 & 1 & 2 & 0 & 0 & 1 & 1 & 3 & 1 & 1 & 0 & 1 \\
\hline 2063229 & 0 & 1 & 1 & 4 & 1 & 1 & 3 & 2 & 1 & 1 & 0 & 0 & 1 & 1 & 1 & 1 & 0 & 0 & 0 \\
\hline 2063230 & 1 & 4 & 1 & 1 & 1 & 2 & 1 & 1 & 4 & 4 & 0 & 0 & 1 & 1 & 5 & 5 & 1 & 1 & 1 \\
\hline 206331 & 1 & 3 & 1 & 1 & 1 & 2 & 1 & 1 & 3 & 4 & 0 & 0 & 1 & 1 & 3 & 1 & 1 & 0 & 1 \\
\hline 206332 & 1 & 3 & 1 & 1 & 1 & 2 & 1 & 2 & 3 & 4 & 0 & 0 & 1 & 1 & 3 & 1 & 1 & 0 & 1 \\
\hline 206333 & 0 & 2 & 1 & 2 & 1 & 2 & 1 & 2 & 3 & 4 & 0 & 0 & 1 & 1 & 2 & 1 & 1 & 0 & 1 \\
\hline 206334 & 1 & 5 & 1 & 3 & 1 & 2 & 1 & 1 & 3 & 3 & 0 & 0 & 1 & 0 & 5 & 5 & 1 & 1 & 1 \\
\hline 206335 & 1 & 3 & 1 & 2 & 1 & 2 & 1 & 2 & 1 & 2 & 0 & 0 & 1 & 1 & 1 & 1 & 0 & 0 & 0 \\
\hline 206336 & 1 & 2 & 1 & 2 & 1 & 2 & 2 & 2 & 1 & 1 & 0 & 0 & 1 & 1 & 1 & 1 & 0 & 0 & 0 \\
\hline 206337 & 0 & 3 & 1 & 1 & 1 & 2 & 1 & 2 & 1 & 1 & 0 & 0 & 1 & 1 & 1 & 1 & 0 & 0 & 0 \\
\hline 206338 & 0 & 4 & 1 & 2 & 1 & 1 & 1 & 2 & 1 & 1 & 0 & 0 & 1 & 1 & 1 & 1 & 0 & 0 & 0 \\
\hline 206339 & 1 & 3 & 1 & 2 & 1 & 2 & 2 & 2 & 1 & 2 & 0 & 0 & 1 & 1 & 1 & 1 & 0 & 0 & 0 \\
\hline 2063310 & 0 & 2 & 1 & 1 & 1 & 2 & 1 & 2 & 1 & 1 & 0 & 0 & 1 & 1 & 3 & 5 & 1 & 1 & 1 \\
\hline 2063311 & 1 & 5 & 1 & 2 & 1 & 2 & 1 & 1 & 1 & 1 & 0 & 0 & 1 & 1 & 2 & 1 & 1 & 0 & 1 \\
\hline 2063312 & 1 & 2 & 1 & 1 & 1 & 4 & 1 & 1 & 1 & 1 & 0 & 0 & 1 & 1 & 3 & 1 & 1 & 0 & 1 \\
\hline 2063313 & 1 & 2 & 1 & 2 & 1 & 2 & 2 & 1 & 3 & 1 & 0 & 0 & 1 & 1 & 3 & 1 & 1 & 0 & 1 \\
\hline 2063314 & 0 & 4 & 1 & 2 & 1 & 2 & 1 & 1 & 4 & 2 & 0 & 0 & 1 & 1 & 1 & 1 & 0 & 0 & 0 \\
\hline 2063315 & 1 & 4 & 1 & 2 & 1 & 2 & 2 & 1 & 3 & 1 & 0 & 0 & 1 & 1 & 1 & 1 & \begin{tabular}{l|l}
0 \\
\end{tabular} & 0 & 0 \\
\hline 2063316 & 0 & 1 & 1 & 2 & 1 & 2 & 1 & 2 & 3 & 1 & 0 & 0 & 1 & 1 & 2 & 1 & 1 & 0 & 1 \\
\hline 2063317 & 0 & 2 & 1 & 1 & 1 & 2 & 1 & 2 & 3 & 1 & 0 & 0 & 1 & 1 & 1 & 1 & 0 & 0 & 0 \\
\hline 2063318 & 0 & 4 & 1 & 1 & 1 & 2 & 1 & 2 & 3 & 1 & 0 & 0 & 1 & 1 & 1 & 1 & 0 & 0 & 0 \\
\hline 2063319 & 1 & 2 & 1 & 1 & 1 & 2 & 1 & 2 & 3 & 1 & 0 & 0 & 1 & 1 & 1 & 1 & 0 & 0 & 0 \\
\hline 2063320 & 1 & 3 & 1 & 2 & 1 & 2 & 2 & 2 & 3 & 1 & 0 & 0 & 1 & 1 & 3 & 1 & 1 & 0 & 1 \\
\hline 2063321 & 0 & 3 & 1 & 4 & 1 & 2 & 1 & 2 & 3 & 2 & 0 & 0 & 1 & 1 & 1 & 1 & \begin{tabular}{l|l}
0 \\
\end{tabular} & 0 & 0 \\
\hline 2063322 & 0 & 1 & 1 & 2 & 1 & 2 & 1 & 1 & 3 & 1 & 0 & 0 & 1 & 1 & 2 & 1 & 1 & 0 & 1 \\
\hline 2063323 & 1 & 2 & 1 & 4 & 1 & 2 & 2 & 2 & 3 & 3 & 0 & 0 & 1 & 1 & 2 & 1 & 1 & 0 & 1 \\
\hline 2063324 & 1 & 4 & 1 & 2 & 1 & 2 & 1 & 1 & 3 & 3 & 0 & 0 & 1 & 1 & 3 & 1 & 1 & 0 & 1 \\
\hline 2063325 & 0 & 5 & 1 & 2 & 1 & 2 & 1 & 1 & 3 & 3 & 0 & 0 & 1 & 1 & 2 & 1 & 1 & 0 & 1 \\
\hline 2063326 & 1 & 5 & 1 & 1 & 1 & 4 & 1 & 1 & 3 & 4 & 0 & 0 & 1 & 1 & 2 & 1 & 1 & 0 & 1 \\
\hline 2063327 & 0 & 5 & 1 & 2 & 1 & 2 & 1 & 1 & 1 & 1 & 0 & 0 & 1 & 1 & 1 & 1 & 0 & 0 & 0 \\
\hline 2063328 & 1 & 3 & 1 & 1 & 1 & 2 & 1 & 2 & 1 & 1 & 0 & 0 & 1 & 1 & 1 & 1 & \begin{tabular}{l|l}
0 \\
\end{tabular} & 0 & 0 \\
\hline 2063329 & 0 & 2 & 1 & 1 & 1 & 2 & 2 & 2 & 3 & 1 & 0 & 0 & 1 & 1 & 2 & 1 & 1 & 0 & 1 \\
\hline 2063330 & 0 & 1 & 1 & 2 & 1 & 2 & 1 & 1 & 3 & 1 & 0 & 0 & 1 & 1 & 1 & 1 & 0 & 0 & 0 \\
\hline 307341 & 1 & 5 & 3 & 4 & 4 & 5 & 3 & 2 & 4 & 1 & 1 & 1 & 1 & 1 & 1 & 1 & 0 & 0 & 0 \\
\hline 307342 & 1 & 5 & 3 & 5 & 4 & 5 & 2 & 1 & 4 & 1 & 1 & 1 & 1 & 1 & 1 & 1 & 0 & 0 & 0 \\
\hline 307343 & 0 & 2 & 3 & 3 & 4 & 1 & 1 & 2 & 4 & 2 & 0 & 0 & 1 & 1 & 1 & 1 & 0 & 0 & 0 \\
\hline 307344 & 1 & 4 & 3 & 2 & 4 & 2 & 2 & 1 & 4 & 1 & 0 & 0 & 1 & 1 & 1 & 1 & 0 & 0 & 0 \\
\hline 307345 & 1 & 4 & 3 & 3 & 4 & 2 & 2 & 2 & 4 & 1 & 0 & 0 & 1 & 1 & 1 & 1 & 0 & 0 & 0 \\
\hline 307346 & 1 & 4 & 3 & 5 & 4 & 5 & 2 & 2 & 4 & 1 & 1 & 1 & 1 & 1 & 1 & 1 & 0 & 0 & 0 \\
\hline 307347 & 0 & 3 & 3 & 4 & 4 & 1 & 2 & 2 & 4 & 3 & 1 & 1 & 1 & 1 & 1 & 1 & 0 & 0 & 0 \\
\hline 307348 & 1 & 2 & 3 & 4 & 4 & 5 & 1 & 2 & 4 & 3 & 0 & 0 & 1 & 1 & 1 & 1 & 0 & 0 & 0 \\
\hline 307349 & 1 & 3 & 3 & 3 & 4 & 2 & 2 & 1 & 4 & 3 & 0 & 0 & 1 & 1 & 1 & 1 & 0 & 0 & 0 \\
\hline 3073410 & 0 & 2 & 3 & 3 & 4 & 1 & 1 & 2 & 4 & 3 & 0 & 0 & 1 & 1 & 1 & 1 & 0 & 0 & $\overline{0}$ \\
\hline 3073411 & 1 & 1 & 3 & 3 & 4 & 2 & 2 & 2 & 4 & 3 & 0 & 0 & 1 & 1 & 1 & 1 & 0 & 0 & 0 \\
\hline 3073412 & 0 & 1 & 3 & 5 & 4 & 5 & 3 & 1 & 4 & 3 & 1 & 1 & 1 & 1 & 1 & 1 & 0 & 0 & \\
\hline
\end{tabular}




\begin{tabular}{|c|c|c|c|c|c|c|c|c|c|c|c|c|c|c|c|c|c|c|c|}
\hline 3073413 & 0 & 1 & 3 & 2 & 4 & 1 & 1 & 2 & 4 & 3 & 0 & 0 & 1 & 1 & 1 & 1 & 0 & 0 & 0 \\
\hline 3073414 & 1 & 5 & 3 & 3 & 4 & 2 & 2 & 2 & 4 & 3 & 0 & 0 & 0 & 99 & 99 & 99 & 99 & 99 & 99 \\
\hline 3073415 & 1 & 4 & 3 & 2 & 4 & 2 & 1 & 2 & 4 & 3 & 0 & 0 & 1 & 1 & 1 & 1 & 0 & 0 & 0 \\
\hline 3073416 & 1 & 2 & 3 & 3 & 4 & 2 & 1 & 1 & 4 & 3 & 0 & 0 & 1 & 1 & 1 & 1 & 0 & 0 & 0 \\
\hline 3073417 & 1 & 2 & 3 & 3 & 4 & 2 & 1 & 2 & 4 & 3 & 0 & 0 & 1 & 1 & 1 & 1 & 0 & 0 & 0 \\
\hline 3073418 & 1 & 4 & 3 & 2 & 4 & 2 & 1 & 1 & 4 & 3 & 0 & 0 & 0 & 99 & 99 & 99 & 99 & 99 & 99 \\
\hline 3073419 & 1 & 2 & 3 & 3 & 4 & 2 & 1 & 2 & 4 & 3 & 0 & 0 & 1 & 1 & 1 & 1 & 0 & 0 & 0 \\
\hline 3073420 & 1 & 2 & 3 & 2 & 4 & 2 & 1 & 2 & 4 & 3 & 0 & 0 & 1 & 1 & 1 & 1 & 0 & 0 & 0 \\
\hline 3073421 & 1 & 3 & 3 & 2 & 4 & 2 & 2 & 1 & 4 & 3 & 0 & 0 & 1 & 1 & 1 & 1 & 0 & 0 & 0 \\
\hline 3073422 & 1 & 3 & 3 & 2 & 4 & 2 & 2 & 2 & 4 & 3 & 0 & 0 & 1 & 1 & 1 & 1 & 0 & 0 & 0 \\
\hline 3073423 & 1 & 4 & 3 & 4 & 4 & 5 & 2 & 2 & 4 & 3 & 0 & 0 & 1 & 1 & 1 & 4 & 0 & 1 & 1 \\
\hline 3073424 & 1 & 2 & 3 & 5 & 4 & 5 & 3 & 2 & 4 & 3 & 1 & 1 & 1 & 1 & 1 & 1 & 0 & 0 & 0 \\
\hline 3073425 & 1 & 3 & 3 & 2 & 4 & 2 & 1 & 2 & 4 & 3 & 0 & 0 & 1 & 1 & 1 & 1 & 0 & 0 & 0 \\
\hline 3073426 & 1 & 4 & 3 & 2 & 4 & 2 & 1 & 1 & 4 & 3 & 0 & 0 & 1 & 1 & 1 & 1 & 0 & 0 & 0 \\
\hline 3073427 & 1 & 4 & 3 & 2 & 4 & 2 & 1 & 2 & 4 & 3 & 0 & 0 & 1 & 1 & 1 & 1 & 0 & 0 & 0 \\
\hline 3073428 & 1 & 1 & 3 & 5 & 4 & 5 & 3 & 2 & 4 & 3 & 1 & 1 & 1 & 1 & 1 & 1 & 0 & 0 & 0 \\
\hline 3073429 & 1 & 2 & 3 & 4 & 4 & 2 & 2 & 1 & 4 & 3 & 0 & 0 & 1 & 1 & 1 & 1 & 0 & 0 & 0 \\
\hline 3073430 & 1 & 4 & 3 & 4 & 4 & 2 & 1 & 1 & 4 & 3 & 0 & 0 & 0 & 99 & 99 & 99 & 99 & 99 & 99 \\
\hline 3073431 & 1 & 4 & 3 & 2 & 4 & 2 & 1 & 1 & 4 & 3 & 0 & 0 & 1 & 1 & 1 & 1 & 0 & 0 & 0 \\
\hline 3073432 & 1 & 2 & 3 & 5 & 4 & 5 & 2 & 2 & 4 & 3 & 1 & 1 & 1 & 1 & 1 & 1 & 0 & 0 & 0 \\
\hline 307351 & 0 & 2 & 3 & 5 & 4 & 5 & 3 & 2 & 4 & 2 & 1 & 1 & 1 & 1 & 1 & 1 & 0 & 0 & 0 \\
\hline 307352 & 1 & 2 & 3 & 2 & 4 & 2 & 1 & 2 & 4 & 2 & 0 & 0 & 1 & 1 & 1 & 1 & 0 & 0 & 0 \\
\hline 307353 & 1 & 2 & 3 & 2 & 4 & 2 & 1 & 2 & 4 & 2 & 0 & 0 & 1 & 1 & 1 & 1 & 0 & 0 & 0 \\
\hline 307354 & 0 & 4 & 3 & 2 & 4 & 1 & 1 & 2 & 4 & 2 & 0 & 0 & 1 & 1 & 1 & 1 & 0 & 0 & 0 \\
\hline 307355 & 1 & 3 & 3 & 3 & 4 & 2 & 2 & 2 & 4 & 2 & 0 & 0 & 1 & 1 & 1 & 1 & 0 & 0 & 0 \\
\hline 307356 & 0 & 2 & 3 & 2 & 4 & 1 & 1 & 2 & 4 & 2 & 0 & 0 & 1 & 1 & 1 & 1 & 0 & 0 & 0 \\
\hline 307357 & 0 & 5 & 3 & 2 & 4 & 1 & 1 & 1 & 4 & 2 & 0 & 0 & 1 & 1 & 1 & 1 & 0 & 0 & 0 \\
\hline 307358 & 0 & 4 & 3 & 2 & 4 & 1 & 1 & 1 & 4 & 2 & 0 & 0 & 1 & 1 & 1 & 1 & 0 & 0 & 0 \\
\hline 307359 & 0 & 2 & 3 & 3 & 4 & 1 & 1 & 1 & 4 & 2 & 0 & 0 & 1 & 1 & 1 & 1 & 0 & 0 & 0 \\
\hline 3073510 & 1 & 2 & 3 & 3 & 4 & 2 & 1 & 2 & 4 & 2 & 0 & 0 & 1 & 1 & 1 & 1 & 0 & 0 & 0 \\
\hline 3073511 & 1 & 5 & 3 & 2 & 4 & 2 & 2 & 1 & 4 & 2 & 0 & 0 & 1 & 1 & 1 & 1 & 0 & 0 & 0 \\
\hline 3073512 & 0 & 1 & 3 & 4 & 4 & 1 & 2 & 2 & 4 & 2 & 1 & 1 & 1 & 1 & 1 & 1 & 0 & 0 & 0 \\
\hline 3073513 & 1 & 3 & 3 & 2 & 4 & 2 & 1 & 2 & 4 & 2 & 0 & 0 & 1 & 1 & 1 & 1 & 0 & 0 & 0 \\
\hline 3073514 & 1 & 3 & 3 & 3 & 4 & 2 & 1 & 2 & 4 & 2 & 0 & 0 & 1 & 1 & 1 & 1 & 0 & 0 & 0 \\
\hline 3073515 & 0 & 3 & 3 & 2 & 4 & 1 & 1 & 2 & 4 & 2 & 0 & 0 & 1 & 1 & 1 & 1 & 0 & 0 & 0 \\
\hline 3073516 & 0 & 3 & 3 & 2 & 4 & 1 & 1 & 2 & 4 & 2 & 0 & 0 & 1 & 1 & 1 & 1 & 0 & 0 & 0 \\
\hline 3073517 & 0 & 4 & 3 & 2 & 4 & 1 & 1 & 2 & 4 & 2 & 0 & 0 & 1 & 1 & 1 & 1 & 0 & 0 & 0 \\
\hline 3073518 & 0 & 2 & 3 & 3 & 4 & 1 & 1 & 1 & 4 & 2 & 0 & 0 & 1 & 1 & 1 & 1 & 0 & 0 & 0 \\
\hline 3073519 & 0 & 2 & 3 & 3 & 4 & 1 & 1 & 2 & 4 & 2 & 0 & 0 & 1 & 1 & 1 & 1 & 0 & 0 & 0 \\
\hline 3073520 & 1 & 2 & 3 & 2 & 4 & 2 & 1 & 2 & 4 & 2 & 0 & 0 & 1 & 1 & 1 & 1 & 0 & 0 & 0 \\
\hline 3073521 & 0 & 2 & 3 & 5 & 4 & 5 & 2 & 1 & 4 & 1 & 1 & 1 & 1 & 1 & 1 & 1 & 0 & 0 & 0 \\
\hline 3073522 & 1 & 2 & 3 & 5 & 4 & 5 & 2 & 1 & 4 & 1 & 1 & 1 & 1 & 1 & 1 & 1 & 0 & 0 & 0 \\
\hline 3073523 & 0 & 3 & 3 & 3 & 4 & 1 & 2 & 2 & 4 & 2 & 0 & 0 & 0 & 99 & 99 & 99 & 99 & 99 & 99 \\
\hline 3073524 & 1 & 3 & 3 & 5 & 4 & 5 & 3 & 2 & 4 & 2 & 1 & 1 & 1 & 1 & 1 & 1 & 0 & 0 & 0 \\
\hline 3073525 & 1 & 2 & 3 & 5 & 4 & 5 & 3 & 1 & 4 & 1 & 1 & 1 & 1 & 1 & 1 & 1 & 0 & 0 & 0 \\
\hline 3073526 & 1 & 4 & 3 & 4 & 4 & 5 & 3 & 1 & 4 & 2 & 1 & 1 & 1 & 1 & 1 & 1 & 0 & 0 & 0 \\
\hline 3073527 & 1 & 1 & 3 & 5 & 4 & 5 & 2 & 1 & 4 & 1 & 1 & 1 & 1 & 1 & 1 & 1 & 0 & 0 & 0 \\
\hline 3073528 & 1 & 3 & 3 & 4 & 4 & 5 & 3 & 2 & 4 & 2 & 1 & 1 & 1 & 1 & 1 & 1 & 0 & 0 & 0 \\
\hline 3073529 & 1 & 2 & 3 & 5 & 4 & 5 & 3 & 1 & 4 & 1 & 1 & 1 & 1 & 1 & 1 & 1 & 0 & 0 & 0 \\
\hline 3073530 & 1 & 2 & 3 & 2 & 4 & 2 & 2 & 1 & 4 & 1 & 0 & 0 & 1 & 1 & 1 & 1 & 0 & 0 & 0 \\
\hline 3073531 & 1 & 2 & 3 & 3 & 4 & 2 & 2 & 2 & 4 & 1 & 0 & 0 & 1 & 1 & 1 & 1 & 0 & 0 & 0 \\
\hline 3073532 & 1 & 5 & 3 & 4 & 4 & 5 & 2 & 1 & 4 & 1 & 1 & 0 & 1 & 1 & 1 & 1 & 0 & 0 & 0 \\
\hline 3073533 & 1 & 2 & 3 & 5 & 4 & 5 & 2 & 1 & 4 & 1 & 1 & 1 & 1 & 1 & 1 & 1 & 0 & 0 & 0 \\
\hline 307361 & 1 & 2 & 3 & 4 & 4 & 2 & 2 & 2 & 3 & 1 & 1 & 1 & 1 & 1 & 1 & 1 & 0 & 0 & 0 \\
\hline 307362 & 1 & 2 & 3 & 5 & 4 & 5 & 3 & 1 & 3 & 1 & 1 & 1 & 1 & 1 & 1 & 1 & 0 & 0 & 0 \\
\hline 307363 & 1 & 5 & 3 & 5 & 4 & 5 & 2 & 1 & 3 & 1 & 1 & 1 & 1 & 1 & 3 & 1 & 1 & 0 & \\
\hline
\end{tabular}




\begin{tabular}{|c|c|c|c|c|c|c|c|c|c|c|c|c|c|c|c|c|c|c|c|}
\hline 307364 & 1 & 5 & 3 & 2 & 4 & 2 & 2 & 1 & 3 & 1 & 0 & 0 & 1 & 1 & 1 & 1 & 0 & 0 & 0 \\
\hline 307365 & 0 & 3 & 3 & 3 & 4 & 1 & 2 & 2 & 3 & 1 & 0 & 0 & 1 & 1 & 1 & 1 & 0 & 0 & 0 \\
\hline 307366 & 1 & 4 & 3 & 4 & 4 & 2 & 2 & 2 & 3 & 1 & 0 & 0 & 1 & 1 & 3 & 1 & 1 & 0 & 1 \\
\hline 307367 & 1 & 5 & 3 & 2 & 4 & 2 & 1 & 1 & 3 & 4 & 0 & 0 & 1 & 1 & 1 & 1 & 0 & 0 & 0 \\
\hline 307368 & 1 & 4 & 3 & 5 & 2 & 5 & 3 & 2 & 3 & 1 & 1 & 1 & 1 & 1 & 1 & 2 & 0 & 0 & 0 \\
\hline 307369 & 0 & 4 & 3 & 5 & 4 & 5 & 3 & 2 & 3 & 1 & 1 & 1 & 1 & 1 & 1 & 1 & 0 & 0 & 0 \\
\hline 3073610 & 0 & 2 & 3 & 2 & 4 & 1 & 2 & 1 & 3 & 1 & 0 & 0 & 1 & 1 & 4 & 1 & 1 & 0 & 1 \\
\hline 3073611 & 0 & 4 & 3 & 2 & 4 & 2 & 2 & 1 & 3 & 1 & 0 & 0 & 1 & 1 & 1 & 1 & 0 & 0 & 0 \\
\hline 3073612 & 1 & 3 & 3 & 5 & 4 & 5 & 3 & 1 & 3 & 2 & 0 & 0 & 1 & 1 & 1 & 1 & 0 & 0 & 0 \\
\hline 3073613 & 0 & 2 & 3 & 4 & 4 & 5 & 2 & 1 & 3 & 2 & 1 & 0 & 1 & 1 & 3 & 1 & 1 & 0 & 1 \\
\hline 3073614 & 1 & 5 & 3 & 2 & 4 & 2 & 1 & 1 & 3 & 2 & 0 & 0 & 1 & 1 & 3 & 1 & 1 & 0 & 1 \\
\hline 3073615 & 0 & 3 & 3 & 4 & 4 & 5 & 3 & 1 & 3 & 2 & 1 & 1 & 1 & 1 & 1 & 2 & 0 & 0 & 0 \\
\hline 3073616 & 1 & 4 & 3 & 2 & 4 & 2 & 1 & 2 & 3 & 2 & 0 & 0 & 1 & 1 & 4 & 1 & 1 & 0 & 1 \\
\hline 3073617 & 1 & 4 & 3 & 5 & 4 & 5 & 3 & 1 & 3 & 2 & 1 & 1 & 1 & 1 & 1 & 1 & 0 & 0 & 0 \\
\hline 3073618 & 1 & 2 & 3 & 4 & 4 & 5 & 3 & 1 & 3 & 2 & 0 & 0 & 1 & 1 & 3 & 1 & 1 & 0 & 1 \\
\hline 3073619 & 1 & 5 & 3 & 2 & 4 & 2 & 2 & 1 & 3 & 2 & 0 & 0 & 1 & 1 & 3 & 1 & 1 & 0 & 1 \\
\hline 3073620 & 1 & 2 & 3 & 2 & 4 & 2 & 2 & 2 & 3 & 2 & 0 & 0 & 1 & 1 & 1 & 1 & 0 & 0 & 0 \\
\hline 3073621 & 1 & 2 & 3 & 4 & 4 & 2 & 2 & 2 & 3 & 2 & 0 & 0 & 1 & 1 & 3 & 1 & 1 & 0 & 1 \\
\hline 3073622 & 0 & 3 & 3 & 3 & 4 & 2 & 2 & 2 & 3 & 4 & 0 & 0 & 1 & 1 & 3 & 1 & 1 & 0 & 1 \\
\hline 3073623 & 1 & 5 & 3 & 4 & 4 & 2 & 2 & 1 & 3 & 4 & 0 & 0 & 1 & 1 & 3 & 1 & 1 & 0 & 1 \\
\hline 3073624 & 1 & 4 & 3 & 3 & 4 & 2 & 1 & 2 & 3 & 4 & 0 & 0 & 1 & 1 & 3 & 1 & 1 & 0 & 1 \\
\hline 3073625 & 0 & 5 & 3 & 2 & 4 & 1 & 2 & 1 & 3 & 4 & 0 & 0 & 1 & 1 & 3 & 1 & 1 & 0 & 1 \\
\hline 3073626 & 1 & 4 & 3 & 4 & 4 & 2 & 2 & 2 & 3 & 4 & 0 & 0 & 1 & 1 & 4 & 1 & 1 & 0 & 1 \\
\hline 3073627 & 0 & 3 & 3 & 2 & 4 & 1 & 1 & 1 & 3 & 4 & 0 & 0 & 1 & 1 & 1 & 4 & 0 & 1 & 1 \\
\hline 3073628 & 1 & 5 & 3 & 2 & 4 & 2 & 2 & 2 & 3 & 4 & 0 & 0 & 1 & 1 & 4 & 1 & 1 & 0 & 1 \\
\hline 3073629 & 1 & 2 & 3 & 3 & 4 & 2 & 2 & 2 & 3 & 4 & 0 & 0 & 1 & 1 & 3 & 1 & 1 & 0 & 1 \\
\hline 3073630 & 1 & 4 & 3 & 3 & 4 & 2 & 1 & 2 & 3 & 2 & 0 & 0 & 1 & 1 & 4 & 1 & 1 & 0 & 1 \\
\hline 307371 & 0 & 1 & 3 & 4 & 4 & 1 & 2 & 1 & 1 & 3 & 0 & 0 & 1 & 1 & 1 & 1 & 0 & 0 & 0 \\
\hline 307372 & 0 & 2 & 3 & 4 & 4 & 1 & 2 & 1 & 1 & 3 & 0 & 0 & 1 & 1 & 1 & 1 & 0 & 0 & 0 \\
\hline 307373 & 0 & 2 & 3 & 2 & 4 & 1 & 2 & 2 & 1 & 2 & 0 & 0 & 1 & 1 & 1 & 1 & 0 & 0 & 0 \\
\hline 307374 & 0 & 3 & 3 & 2 & 4 & 1 & 2 & 2 & 1 & 1 & 0 & 0 & 0 & 99 & 99 & 99 & 99 & 99 & 99 \\
\hline 307375 & 0 & 2 & 3 & 2 & 4 & 2 & 1 & 2 & 1 & 4 & 0 & 0 & 1 & 1 & 3 & 1 & 1 & 0 & 1 \\
\hline 307376 & 0 & 1 & 3 & 4 & 4 & 2 & 1 & 1 & 1 & 4 & 0 & 0 & 1 & 1 & 4 & 1 & 1 & 0 & 1 \\
\hline 307377 & 1 & 2 & 3 & 2 & 4 & 2 & 2 & 2 & 1 & 1 & 1 & 1 & 1 & 1 & 1 & 1 & 0 & 0 & 0 \\
\hline 307378 & 0 & 5 & 3 & 2 & 4 & 1 & 2 & 1 & 1 & 4 & 0 & 0 & 0 & 99 & 99 & 99 & 99 & 99 & 99 \\
\hline 307379 & 1 & 2 & 3 & 2 & 4 & 2 & 2 & 2 & 1 & 1 & 0 & 0 & 1 & 1 & 1 & 1 & 0 & 0 & 0 \\
\hline 3073710 & 0 & 3 & 3 & 3 & 4 & 1 & 2 & 1 & 1 & 1 & 0 & 0 & 1 & 1 & 1 & 1 & 0 & 0 & 0 \\
\hline 3073711 & 1 & 1 & 3 & 2 & 4 & 2 & 1 & 1 & 1 & 4 & 0 & 0 & 0 & 99 & 99 & 99 & 99 & 99 & 99 \\
\hline 3073712 & 1 & 5 & 3 & 1 & 4 & 2 & 1 & 1 & 1 & 4 & 0 & 0 & 0 & 99 & 99 & 99 & 99 & 99 & 99 \\
\hline 3073713 & 1 & 1 & 3 & 2 & 2 & 2 & 1 & 1 & 1 & 3 & 0 & 0 & 1 & 1 & 3 & 1 & 1 & 0 & 1 \\
\hline 3073714 & 1 & 4 & 3 & 4 & 4 & 2 & 2 & 2 & 1 & 3 & 0 & 0 & 1 & 1 & 2 & 1 & 1 & 0 & 1 \\
\hline 3073715 & 0 & 2 & 3 & 3 & 4 & 3 & 3 & 2 & 1 & 2 & 1 & 0 & 1 & 1 & 1 & 1 & 0 & 0 & 0 \\
\hline 3073716 & 1 & 2 & 3 & 2 & 4 & 2 & 2 & 1 & 4 & 3 & 0 & 0 & 1 & 1 & 1 & 1 & 0 & 0 & 0 \\
\hline 3073717 & 0 & 1 & 3 & 3 & 4 & 1 & 1 & 1 & 4 & 4 & 0 & $\overline{0}$ & 1 & 1 & 2 & 1 & 1 & 0 & 1 \\
\hline 3073718 & 0 & 2 & 3 & 3 & 4 & 1 & 2 & 2 & 4 & 4 & 0 & 0 & 0 & 99 & 99 & 99 & 99 & 99 & 99 \\
\hline 3073719 & 1 & 5 & 3 & 2 & 4 & 2 & 1 & 1 & 4 & 4 & 0 & 0 & 0 & 99 & 99 & 99 & 99 & 99 & 99 \\
\hline 3073720 & 0 & 2 & 3 & 3 & 4 & 1 & 1 & 1 & 4 & 4 & 0 & 0 & 1 & 1 & 2 & 1 & 1 & 0 & 1 \\
\hline 3073721 & 1 & 5 & 3 & 2 & 4 & 2 & 2 & 2 & 4 & 3 & 0 & 0 & 1 & 1 & 2 & 1 & 1 & 0 & 1 \\
\hline 3073722 & 0 & 2 & 3 & 2 & 4 & 1 & 1 & 1 & 4 & 4 & 0 & $\overline{0}$ & 1 & 1 & 2 & 2 & 1 & 0 & 1 \\
\hline 3073723 & 0 & 3 & 3 & 2 & 4 & 1 & 2 & 2 & 4 & 4 & 0 & 0 & 1 & 1 & 1 & 1 & 0 & 0 & 0 \\
\hline 3073724 & 0 & 1 & 3 & 2 & 4 & 1 & 2 & 1 & 4 & 2 & 0 & 0 & 1 & 1 & 1 & 1 & 0 & 0 & 0 \\
\hline 3073725 & 0 & 4 & 3 & 3 & 4 & 3 & 2 & 1 & 4 & 3 & 0 & 0 & 1 & 1 & 1 & 1 & 0 & 0 & 0 \\
\hline 3073726 & 1 & 2 & 3 & 2 & 4 & 4 & 2 & 1 & 4 & 3 & 0 & 0 & 0 & 99 & 99 & 99 & 99 & 99 & 99 \\
\hline 3073727 & 1 & 2 & 3 & 2 & 4 & 4 & 2 & 2 & 4 & 3 & 0 & $\overline{0}$ & 1 & 1 & 1 & 1 & 0 & 0 & 0 \\
\hline 3073728 & 0 & 1 & 3 & 2 & 4 & 1 & 1 & 2 & 4 & 2 & 0 & 0 & 0 & 99 & 99 & 99 & 99 & 99 & 99 \\
\hline 3073729 & 0 & 2 & 3 & 3 & 4 & 1 & 2 & 2 & 4 & 3 & 0 & 0 & 0 & 99 & 99 & 99 & 99 & 99 & 99 \\
\hline
\end{tabular}




\begin{tabular}{|c|c|c|c|c|c|c|c|c|c|c|c|c|c|c|c|c|c|c|c|}
\hline 3073730 & 0 & 1 & 3 & 2 & 3 & 1 & 1 & 1 & 4 & 2 & 0 & 0 & 0 & 99 & 99 & 99 & 99 & 99 & 99 \\
\hline 307381 & 0 & 2 & 3 & 2 & 4 & 2 & 2 & 1 & 4 & 1 & 0 & 0 & 1 & 1 & 1 & 4 & 0 & 1 & 1 \\
\hline 307382 & 1 & 4 & 3 & 3 & 4 & 2 & 2 & 2 & 4 & 1 & 0 & 0 & 1 & 1 & 1 & 4 & 0 & 1 & 1 \\
\hline 307383 & 0 & 2 & 3 & 3 & 4 & 2 & 2 & 2 & 4 & 1 & 0 & 0 & 1 & 1 & 1 & 4 & 0 & 1 & 1 \\
\hline 307384 & 1 & 3 & 3 & 2 & 4 & 2 & 2 & 2 & 4 & 1 & 0 & 0 & 1 & 1 & 1 & 4 & 0 & 1 & 1 \\
\hline 307385 & 0 & 1 & 3 & 4 & 4 & 2 & 2 & 2 & 4 & 1 & 0 & 0 & 1 & 1 & 1 & 1 & 0 & 0 & 0 \\
\hline 307386 & 1 & 3 & 3 & 2 & 4 & 2 & 2 & 2 & 4 & 1 & 0 & 0 & 1 & 1 & 1 & 4 & 0 & 1 & 1 \\
\hline 307387 & 0 & 1 & 3 & 3 & 4 & 2 & 2 & 2 & 4 & 1 & 0 & 0 & 1 & 1 & 1 & 4 & 0 & 1 & 1 \\
\hline 307388 & 1 & 3 & 3 & 3 & 4 & 2 & 2 & 1 & 4 & 1 & 0 & 0 & 1 & 1 & 3 & 1 & 1 & 0 & 1 \\
\hline 307389 & 1 & 3 & 3 & 4 & 4 & 2 & 2 & 1 & 4 & 1 & 0 & 0 & 1 & 1 & 3 & 1 & 1 & 0 & 1 \\
\hline 3073810 & 0 & 2 & 3 & 4 & 4 & 3 & 2 & 1 & 4 & 1 & 1 & 0 & 1 & 1 & 1 & 1 & 0 & 0 & 0 \\
\hline 3073811 & 0 & 2 & 3 & 5 & 4 & 5 & 2 & 2 & 4 & 1 & 1 & 0 & 1 & 1 & 1 & 1 & 0 & 0 & 0 \\
\hline 3073812 & 1 & 2 & 3 & 3 & 4 & 2 & 2 & 2 & 4 & 1 & 0 & 0 & 1 & 1 & 1 & 1 & 0 & 0 & 0 \\
\hline 3073813 & 0 & 3 & 3 & 2 & 4 & 2 & 2 & 1 & 4 & 1 & 0 & 0 & 1 & 1 & 1 & 4 & 0 & 1 & 1 \\
\hline 3073814 & 1 & 2 & 3 & 5 & 4 & 5 & 2 & 2 & 4 & 1 & 0 & 0 & 1 & 1 & 3 & 1 & 1 & 0 & 1 \\
\hline 3073815 & 0 & 2 & 3 & 5 & 4 & 5 & 3 & 1 & 4 & 1 & 1 & 0 & 1 & 1 & 3 & 1 & 1 & 0 & 1 \\
\hline 3073816 & 1 & 2 & 3 & 2 & 4 & 2 & 1 & 2 & 4 & 3 & 0 & 0 & 1 & 1 & 1 & 1 & 0 & 0 & 0 \\
\hline 3073817 & 0 & 2 & 3 & 5 & 4 & 5 & 3 & 1 & 4 & 1 & 1 & 0 & 1 & 1 & 1 & 1 & 0 & 0 & 0 \\
\hline 3073818 & 1 & 4 & 3 & 3 & 4 & 2 & 2 & 2 & 4 & 2 & 0 & 0 & 1 & 1 & 1 & 1 & 0 & 0 & 0 \\
\hline 3073819 & 0 & 1 & 3 & 4 & 4 & 3 & 3 & 1 & 4 & 1 & 1 & 0 & 1 & 1 & 1 & 1 & 0 & 0 & 0 \\
\hline 3073820 & 1 & 3 & 3 & 4 & 4 & 2 & 2 & 2 & 4 & 2 & 0 & 0 & 1 & 1 & 3 & 1 & 1 & 0 & 1 \\
\hline 3073821 & 0 & 2 & 3 & 5 & 4 & 5 & 2 & 1 & 4 & 4 & 0 & 0 & 1 & 1 & 1 & 1 & 0 & 0 & 0 \\
\hline 3073822 & 1 & 4 & 3 & 2 & 4 & 2 & 2 & 2 & 4 & 3 & 0 & 0 & 1 & 1 & 1 & 1 & 0 & 0 & 0 \\
\hline 3073823 & 0 & 2 & 3 & 3 & 4 & 2 & 2 & 2 & 4 & 2 & 0 & 0 & 1 & 1 & 1 & 1 & 0 & 0 & 0 \\
\hline 3073824 & 1 & 4 & 3 & 2 & 4 & 2 & 2 & 2 & 4 & 3 & 0 & 0 & 1 & 1 & 1 & 1 & 0 & 0 & 0 \\
\hline 3073825 & 0 & 1 & 3 & 4 & 4 & 2 & 2 & 1 & 4 & 3 & 0 & 0 & 1 & 1 & 1 & 1 & 0 & 0 & 0 \\
\hline 3073826 & 0 & 4 & 3 & 2 & 4 & 1 & 2 & 1 & 4 & 3 & 0 & 0 & 1 & 1 & 1 & 1 & 0 & 0 & 0 \\
\hline 3073827 & 1 & 4 & 3 & 2 & 2 & 2 & 2 & 2 & 4 & 3 & 0 & 0 & 1 & 1 & 1 & 1 & 0 & 0 & 0 \\
\hline 3073828 & 1 & 4 & 3 & 4 & 4 & 5 & 3 & 2 & 4 & 2 & 1 & 1 & 1 & 1 & 1 & 1 & 0 & 0 & 0 \\
\hline 3073829 & 0 & 2 & 3 & 2 & 4 & 2 & 2 & 2 & 4 & 4 & 0 & 0 & 1 & 1 & 1 & 4 & 0 & 1 & 1 \\
\hline 3073830 & 1 & 4 & 3 & 2 & 4 & 2 & 2 & 2 & 4 & 3 & 0 & 0 & 1 & 1 & 1 & 1 & 0 & 0 & 0 \\
\hline 307391 & 1 & 3 & 3 & 4 & 4 & 5 & 2 & 2 & 1 & 3 & 1 & 1 & 1 & 1 & 1 & 1 & 0 & 0 & 0 \\
\hline 307392 & 1 & 1 & 3 & 4 & 4 & 2 & 2 & 2 & 2 & 3 & 0 & 0 & 1 & 1 & 1 & 6 & 0 & 1 & 1 \\
\hline 307393 & 1 & 2 & 3 & 2 & 4 & 2 & 2 & 2 & 2 & 3 & 0 & 0 & 1 & 1 & 2 & 1 & 1 & 0 & 1 \\
\hline 307394 & 0 & 2 & 3 & 2 & 4 & 1 & 2 & 2 & 2 & 3 & 0 & 0 & 0 & 99 & 99 & 99 & 99 & 99 & 99 \\
\hline 307395 & 1 & 1 & 3 & 4 & 4 & 2 & 2 & 1 & 2 & 3 & 0 & 0 & 0 & 99 & 99 & 99 & 99 & 99 & 99 \\
\hline 307396 & 0 & 2 & 3 & 2 & 4 & 1 & 2 & 2 & 3 & 2 & 0 & 0 & 0 & 99 & 99 & 99 & 99 & 99 & 99 \\
\hline 307397 & 1 & 3 & 3 & 2 & 4 & 2 & 2 & 2 & 3 & 2 & 0 & 0 & 0 & 99 & 99 & 99 & 99 & 99 & 99 \\
\hline 307398 & 1 & 4 & 3 & 3 & 4 & 2 & 2 & 1 & 2 & 3 & 0 & 0 & 0 & 99 & 99 & 99 & 99 & 99 & 99 \\
\hline 307399 & 0 & 4 & 3 & 3 & 4 & 1 & 1 & 1 & 2 & 2 & 0 & 0 & 1 & 1 & 4 & 1 & 1 & 0 & 1 \\
\hline 3073910 & 1 & 4 & 3 & 2 & 4 & 2 & 2 & 2 & 2 & 2 & 0 & 0 & 1 & 1 & 1 & 1 & 0 & 0 & 0 \\
\hline 3073911 & 1 & 5 & 3 & 3 & 4 & 2 & 1 & 1 & 2 & 2 & 0 & 0 & 1 & 1 & 1 & 1 & 0 & 0 & 0 \\
\hline 3073912 & 0 & 1 & 3 & 5 & 4 & 5 & 2 & 1 & 2 & 1 & 0 & 0 & 1 & 1 & 1 & 1 & 0 & 0 & 0 \\
\hline 3073913 & 1 & 3 & 3 & 5 & 4 & 5 & 2 & 1 & 2 & 1 & 1 & 0 & 1 & 1 & 1 & 1 & 0 & 0 & 0 \\
\hline 3073914 & 0 & 2 & 3 & 2 & 4 & 1 & 1 & 2 & 2 & 2 & 0 & 0 & 0 & 99 & 99 & 99 & 99 & 99 & 99 \\
\hline 3073915 & 0 & 1 & 3 & 3 & 4 & 1 & 2 & 1 & 2 & 1 & 0 & 0 & 0 & 99 & 99 & 99 & 99 & 99 & 99 \\
\hline 3073916 & 0 & 2 & 3 & 2 & 4 & 2 & 1 & 2 & 2 & 2 & 0 & 0 & 0 & 99 & 99 & 99 & 99 & 99 & 99 \\
\hline 3073917 & 0 & 2 & 3 & 2 & 4 & 1 & 1 & 2 & 2 & 1 & 0 & 0 & 0 & 99 & 99 & 99 & 99 & 99 & 99 \\
\hline 3073918 & 1 & 4 & 3 & 2 & 4 & 2 & 1 & 1 & 2 & 2 & 0 & 0 & 0 & 99 & 99 & 99 & 99 & 99 & 99 \\
\hline 3073919 & 1 & 4 & 3 & 2 & 4 & 2 & 1 & 2 & 2 & 1 & 0 & 0 & 0 & 99 & 99 & 99 & 99 & 99 & 99 \\
\hline 3073920 & 0 & 2 & 3 & 2 & 4 & 1 & 2 & 1 & 2 & 2 & 0 & 0 & 0 & 99 & 99 & 99 & 99 & 99 & 99 \\
\hline 3073921 & 1 & 2 & 3 & 2 & 4 & 2 & 1 & 1 & 2 & 2 & 0 & 0 & 1 & 1 & 3 & 1 & 1 & 0 & 1 \\
\hline 3073922 & 1 & 5 & 3 & 2 & 4 & 2 & 1 & 2 & 2 & 2 & 0 & 0 & 1 & 1 & 2 & 1 & 1 & 0 & 1 \\
\hline 3073923 & 1 & 2 & 3 & 2 & 4 & 2 & 1 & 1 & 2 & 2 & 0 & 0 & 0 & 99 & 99 & 99 & 99 & 99 & 99 \\
\hline 3073924 & 0 & 2 & 3 & 2 & 4 & 1 & 2 & 2 & 2 & 2 & 0 & 0 & 0 & 99 & 99 & 99 & 99 & 99 & 99 \\
\hline 3073925 & 1 & 5 & 3 & 2 & 4 & 2 & 1 & 1 & 2 & 2 & 0 & 0 & 0 & 99 & 99 & 99 & 99 & 99 & 99 \\
\hline
\end{tabular}




\begin{tabular}{|c|c|c|c|c|c|c|c|c|c|c|c|c|c|c|c|c|c|c|c|}
\hline 3073926 & 0 & 2 & 3 & 2 & 4 & 1 & 1 & 1 & 2 & 2 & 0 & 0 & 1 & 1 & 2 & 1 & 1 & 0 & 1 \\
\hline 3073927 & 0 & 2 & 3 & 2 & 4 & 1 & 2 & 2 & 2 & 2 & 0 & 0 & 1 & 1 & 2 & 6 & 1 & 1 & 1 \\
\hline 3073928 & 0 & 3 & 3 & 2 & 4 & 1 & 2 & 1 & 4 & 2 & 0 & 0 & 0 & 99 & 99 & 99 & 99 & 99 & 99 \\
\hline 3073929 & 0 & 3 & 3 & 2 & 4 & 2 & 1 & 1 & 2 & 2 & 0 & 0 & 0 & 99 & 99 & 99 & 99 & 99 & 99 \\
\hline 3073930 & 1 & 2 & 3 & 4 & 4 & 5 & 2 & 2 & 2 & 3 & 0 & 0 & 0 & 99 & 99 & 99 & 99 & 99 & 99 \\
\hline 307401 & 0 & 3 & 3 & 3 & 4 & 2 & 2 & 1 & 2 & 4 & 0 & 0 & 1 & 1 & 3 & 1 & 1 & 0 & 1 \\
\hline 307402 & 1 & 3 & 3 & 2 & 4 & 2 & 1 & 1 & 2 & 4 & 0 & 0 & 1 & 1 & 3 & 1 & 1 & 0 & 1 \\
\hline 307403 & 0 & 2 & 3 & 2 & 4 & 2 & 1 & 2 & 2 & 4 & 0 & 0 & 1 & 1 & 1 & 4 & 0 & 1 & 1 \\
\hline 307404 & 1 & 3 & 3 & 3 & 4 & 2 & 2 & 2 & 2 & 4 & 0 & 0 & 1 & 1 & 1 & 4 & 0 & 1 & 1 \\
\hline 307405 & 1 & 3 & 3 & 2 & 4 & 2 & 2 & 2 & 2 & 4 & 0 & 0 & 1 & 1 & 1 & 4 & 0 & 1 & 1 \\
\hline 307406 & 1 & 3 & 3 & 3 & 4 & 2 & 2 & 2 & 2 & 4 & 0 & 0 & 1 & 1 & 1 & 4 & 0 & 1 & 1 \\
\hline 307407 & 0 & 2 & 3 & 2 & 4 & 2 & 2 & 2 & 2 & 4 & 0 & 0 & 1 & 1 & 3 & 1 & 1 & 0 & 1 \\
\hline 307408 & 0 & 1 & 3 & 4 & 4 & 2 & 2 & 2 & 2 & 4 & 0 & 0 & 1 & 1 & 3 & 1 & 1 & 0 & 1 \\
\hline 307409 & 0 & 3 & 3 & 3 & 4 & 2 & 2 & 2 & 2 & 4 & 0 & 0 & 1 & 1 & 1 & 4 & 0 & 1 & 1 \\
\hline 3074010 & 1 & 1 & 3 & 5 & 4 & 5 & 2 & 1 & 2 & 4 & 0 & 0 & 1 & 1 & 1 & 1 & 0 & 0 & 0 \\
\hline 3074011 & 1 & 3 & 3 & 2 & 4 & 2 & 1 & 1 & 2 & 1 & 0 & 0 & 1 & 1 & 3 & 1 & 1 & 0 & 1 \\
\hline 3074012 & 1 & 2 & 3 & 5 & 4 & 5 & 3 & 1 & 2 & 1 & 1 & 0 & 1 & 1 & 3 & 1 & 1 & 0 & 1 \\
\hline 3074013 & 0 & 1 & 3 & 4 & 4 & 2 & 2 & 1 & 2 & 1 & 0 & 0 & 1 & 1 & 3 & 1 & 1 & 0 & 1 \\
\hline 3074014 & 0 & 2 & 3 & 3 & 4 & 2 & 2 & 2 & 2 & 1 & 0 & 0 & 1 & 1 & 1 & 4 & 0 & 1 & 1 \\
\hline 3074015 & 0 & 2 & 3 & 4 & 4 & 2 & 2 & 1 & 2 & 1 & 0 & 0 & 1 & 1 & 1 & 4 & 0 & 1 & 1 \\
\hline 3074016 & 1 & 2 & 3 & 5 & 4 & 5 & 3 & 2 & 2 & 1 & 1 & 1 & 1 & 1 & 1 & 1 & 0 & 0 & 0 \\
\hline 3074017 & 0 & 2 & 3 & 2 & 4 & 2 & 1 & 2 & 2 & 1 & 0 & 0 & 1 & 1 & 1 & 4 & 0 & 1 & 1 \\
\hline 3074018 & 1 & 2 & 3 & 2 & 4 & 2 & 1 & 1 & 2 & 3 & 0 & 0 & 1 & 1 & 1 & 4 & 0 & 1 & 1 \\
\hline 3074019 & 1 & 1 & 3 & 3 & 4 & 2 & 1 & 2 & 2 & 3 & 0 & 0 & 1 & 1 & 1 & 1 & 0 & 0 & 0 \\
\hline 3074020 & 1 & 1 & 3 & 2 & 4 & 2 & 1 & 1 & 2 & 1 & 0 & 0 & 1 & 1 & 1 & 4 & 0 & 1 & 1 \\
\hline 3074021 & 0 & 2 & 3 & 3 & 4 & 2 & 2 & 2 & 2 & 4 & 1 & 0 & 1 & 1 & 1 & 4 & 0 & 1 & 1 \\
\hline 3074022 & 0 & 2 & 3 & 2 & 4 & 2 & 1 & 2 & 2 & 3 & 0 & 0 & 1 & 1 & 1 & 1 & 0 & 0 & 0 \\
\hline 3074023 & 0 & 2 & 3 & 3 & 4 & 2 & 2 & 2 & 2 & 3 & 0 & 0 & 1 & 1 & 1 & 1 & 0 & 0 & 0 \\
\hline 3074024 & 1 & 5 & 3 & 2 & 4 & 2 & 2 & 2 & 2 & 3 & 0 & 0 & 1 & 1 & 1 & 1 & 0 & 0 & 0 \\
\hline 3074025 & 0 & 3 & 3 & 2 & 4 & 2 & 1 & 2 & 2 & 3 & 0 & 0 & 1 & 1 & 1 & 1 & 0 & 0 & 0 \\
\hline 3074026 & 0 & 2 & 3 & 4 & 4 & 2 & 2 & 1 & 2 & 3 & 0 & 0 & 1 & 1 & 3 & 1 & 1 & 0 & 1 \\
\hline 3074027 & 1 & 1 & 3 & 3 & 4 & 2 & 2 & 1 & 2 & 3 & 0 & 0 & 1 & 1 & 1 & 1 & 0 & 0 & 0 \\
\hline 3074028 & 0 & 3 & 3 & 2 & 4 & 2 & 2 & 2 & 2 & 3 & 0 & 0 & 1 & 1 & 1 & 4 & 0 & 1 & 1 \\
\hline 3074029 & 1 & 2 & 3 & 3 & 4 & 2 & 2 & 2 & 2 & 3 & 0 & 0 & 1 & 1 & 1 & 4 & 0 & 1 & 1 \\
\hline 3074030 & 1 & 3 & 3 & 3 & 4 & 2 & 1 & 1 & 2 & 1 & 0 & 0 & 1 & 1 & 1 & 4 & 0 & 1 & 1 \\
\hline 3074031 & 1 & 2 & 3 & 2 & 4 & 2 & 2 & 2 & 2 & 2 & 0 & 0 & 1 & 1 & 1 & 1 & 0 & 0 & 0 \\
\hline 3074032 & 0 & 1 & 3 & 4 & 4 & 2 & 2 & 1 & 2 & 1 & 0 & 0 & 1 & 1 & 1 & 1 & 0 & 0 & 0 \\
\hline 3074033 & 0 & 1 & 3 & 5 & 4 & 5 & 2 & 1 & 2 & 1 & 0 & 0 & 1 & 1 & 1 & 1 & 0 & 0 & 0 \\
\hline 3074034 & 1 & 4 & 3 & 4 & 4 & 2 & 1 & 1 & 2 & 1 & 0 & 0 & 1 & 1 & 3 & 1 & 1 & 0 & 1 \\
\hline 3074035 & 0 & 2 & 3 & 5 & 4 & 5 & 2 & 1 & 2 & 1 & 1 & 0 & 1 & 1 & 3 & 1 & 1 & 0 & 1 \\
\hline 307411 & 0 & 2 & 3 & 2 & 2 & 1 & 2 & 2 & 3 & 2 & 0 & 0 & 1 & 1 & 1 & 1 & 0 & 0 & 0 \\
\hline 307412 & 0 & 4 & 3 & 2 & 4 & 1 & 2 & 1 & 3 & 2 & 0 & 0 & 1 & 1 & 1 & 1 & 0 & 0 & 0 \\
\hline 307413 & 0 & 3 & 3 & 2 & 4 & 1 & 2 & 2 & 3 & 3 & 0 & 0 & 1 & 1 & 1 & 1 & 0 & 0 & 0 \\
\hline 307414 & 0 & 1 & 3 & 4 & 4 & 1 & 1 & 2 & 3 & 3 & 0 & 0 & 1 & 1 & 1 & 1 & 0 & 0 & 0 \\
\hline 307415 & 0 & 2 & 3 & 2 & 4 & 1 & 2 & 2 & 2 & 2 & 0 & 0 & 1 & 1 & 1 & 1 & 0 & 0 & 0 \\
\hline 307416 & 1 & 3 & 3 & 2 & 4 & 2 & 2 & 2 & 2 & 2 & 0 & 0 & 1 & 1 & 2 & 1 & 1 & 0 & 1 \\
\hline 307417 & 0 & 1 & 3 & 2 & 4 & 1 & 2 & 2 & 3 & 2 & 0 & 0 & 1 & 1 & 1 & 1 & 0 & 0 & 0 \\
\hline 307418 & 0 & 2 & 3 & 4 & 4 & 3 & 2 & 1 & 3 & 3 & 0 & 0 & 1 & 1 & 2 & 1 & 1 & 0 & 1 \\
\hline 307419 & 0 & 2 & 3 & 5 & 2 & 5 & 2 & 2 & 3 & 3 & 0 & 0 & 1 & 1 & 2 & 1 & 1 & 0 & 1 \\
\hline 3074110 & 0 & 2 & 3 & 2 & 4 & 1 & 2 & 2 & 3 & 2 & 0 & 0 & 1 & 1 & 2 & 1 & 1 & 0 & 1 \\
\hline 3074111 & 1 & 1 & 3 & 3 & 4 & 2 & 2 & 2 & 3 & 2 & 0 & 0 & 1 & 1 & 2 & 1 & 1 & 0 & 1 \\
\hline 3074112 & 0 & 2 & 3 & 4 & 4 & 1 & 1 & 2 & 3 & 2 & 0 & 0 & 1 & 1 & 2 & 1 & 1 & 0 & 1 \\
\hline 3074113 & 0 & 2 & 3 & 2 & 4 & 1 & 2 & 2 & 3 & 2 & 0 & 0 & 1 & 1 & 1 & 1 & 0 & 0 & 0 \\
\hline 3074114 & 1 & 2 & 3 & 3 & 4 & 2 & 2 & 1 & 3 & 2 & 0 & 0 & 1 & 1 & 1 & 1 & 0 & 0 & 0 \\
\hline 3074115 & 1 & 3 & 3 & 3 & 4 & 4 & 2 & 1 & 3 & 2 & 0 & 0 & 1 & 1 & 1 & 1 & 0 & 0 & 0 \\
\hline 3074116 & 1 & 3 & 3 & 2 & 4 & 3 & 3 & 2 & 3 & 2 & 0 & 0 & 1 & 1 & 1 & 1 & 0 & 0 & \\
\hline
\end{tabular}




\begin{tabular}{|c|c|c|c|c|c|c|c|c|c|c|c|c|c|c|c|c|c|c|c|}
\hline 3074117 & 1 & 3 & 3 & 2 & 4 & 2 & 2 & 2 & 3 & 2 & 0 & 0 & 1 & 1 & 1 & 1 & 0 & 0 & 0 \\
\hline 3074118 & 0 & 3 & 3 & 3 & 4 & 1 & 2 & 2 & 3 & 2 & 0 & 0 & 1 & 1 & 1 & 1 & 0 & 0 & 0 \\
\hline 3074119 & 0 & 4 & 3 & 5 & 4 & 5 & 2 & 1 & 3 & 2 & 0 & 0 & 1 & 1 & 1 & 1 & 0 & 0 & 0 \\
\hline 3074120 & 1 & 5 & 3 & 5 & 4 & 5 & 3 & 2 & 3 & 1 & 1 & 1 & 1 & 1 & 1 & 1 & 0 & 0 & 0 \\
\hline 3074121 & 1 & 4 & 3 & 3 & 4 & 2 & 2 & 1 & 3 & 3 & 0 & 0 & 1 & 1 & 1 & 1 & 0 & 0 & 0 \\
\hline 3074122 & 1 & 3 & 3 & 3 & 4 & 5 & 2 & 1 & 3 & 1 & 0 & 0 & 1 & 1 & 1 & 1 & 0 & 0 & 0 \\
\hline 3074123 & 0 & 4 & 3 & 3 & 4 & 1 & 2 & 1 & 3 & 1 & 0 & 0 & 1 & 1 & 1 & 1 & 0 & 0 & 0 \\
\hline 3074124 & 0 & 5 & 3 & 2 & 4 & 1 & 1 & 1 & 3 & 1 & 0 & 0 & 1 & 1 & 1 & 1 & 0 & 0 & 0 \\
\hline 3074125 & 1 & 5 & 3 & 2 & 4 & 2 & 2 & 2 & 3 & 1 & 0 & 0 & 1 & 1 & 1 & 1 & 0 & 0 & 0 \\
\hline 3074126 & 0 & 2 & 3 & 5 & 4 & 5 & 2 & 1 & 3 & 1 & 0 & 0 & 1 & 1 & 1 & 1 & 0 & 0 & 0 \\
\hline 3074127 & 0 & 5 & 3 & 2 & 4 & 1 & 2 & 2 & 3 & 1 & 0 & 0 & 1 & 1 & 1 & 1 & 0 & 0 & 0 \\
\hline 3074128 & 0 & 5 & 3 & 2 & 4 & 1 & 2 & 2 & 3 & 3 & 0 & 0 & 1 & 1 & 1 & 1 & 0 & 0 & 0 \\
\hline 3074129 & 1 & 4 & 3 & 5 & 4 & 5 & 2 & 2 & 3 & 3 & 1 & 0 & 1 & 1 & 4 & 1 & 1 & 0 & 1 \\
\hline 3074130 & 0 & 2 & 3 & 3 & 4 & 1 & 3 & 2 & 3 & 2 & 1 & 0 & 1 & 1 & 1 & 1 & 0 & 0 & 0 \\
\hline 3074131 & 1 & 5 & 3 & 2 & 4 & 2 & 2 & 2 & 3 & 2 & 0 & 0 & 1 & 1 & 2 & 1 & 1 & 0 & 1 \\
\hline 3074132 & 0 & 2 & 3 & 2 & 4 & 1 & 2 & 2 & 4 & 4 & 0 & 0 & 0 & 99 & 99 & 99 & 99 & 99 & $\overline{99}$ \\
\hline 3074133 & 1 & 4 & 3 & 2 & 4 & 2 & 2 & 1 & 4 & 4 & 0 & 0 & 0 & 99 & 99 & 99 & 99 & 99 & 99 \\
\hline 3074134 & 0 & 2 & 3 & 5 & 4 & 5 & 3 & 1 & 4 & 3 & 1 & 1 & 1 & 1 & 1 & 1 & 0 & 0 & 0 \\
\hline 3074135 & 1 & 5 & 3 & 2 & 4 & 2 & 2 & 1 & 4 & 3 & 0 & 0 & 1 & 1 & 2 & 1 & 1 & 0 & 1 \\
\hline 3074136 & 1 & 5 & 3 & 2 & 4 & 2 & 2 & 1 & 4 & 4 & 0 & 0 & 1 & 1 & 1 & 1 & 0 & 0 & 0 \\
\hline 3074137 & 1 & 5 & 3 & 2 & 4 & 2 & 2 & 2 & 4 & 3 & 0 & 0 & 0 & 99 & 99 & 99 & 99 & 99 & 99 \\
\hline 3074138 & 0 & 3 & 3 & 3 & 4 & 1 & 2 & 1 & 4 & 4 & 0 & 0 & 1 & 1 & 2 & 1 & 1 & 0 & 1 \\
\hline 3074139 & 1 & 3 & 3 & 2 & 4 & 4 & 2 & 1 & 4 & 3 & 0 & 0 & 0 & 99 & 99 & 99 & 99 & 99 & 99 \\
\hline 3074140 & 1 & 5 & 3 & 2 & 4 & 2 & 2 & 2 & 4 & 4 & 0 & 0 & 0 & 99 & 99 & 99 & 99 & 99 & 99 \\
\hline 308421 & 0 & 4 & 3 & 2 & 4 & 2 & 1 & 1 & 4 & 4 & 0 & 0 & 0 & 99 & 99 & 99 & 99 & 99 & 99 \\
\hline 308422 & 1 & 4 & 3 & 2 & 4 & 2 & 2 & 2 & 4 & 4 & 0 & 0 & 1 & 1 & 4 & 1 & 1 & 0 & 1 \\
\hline 308423 & 0 & 3 & 3 & 3 & 4 & 2 & 3 & 2 & 2 & 4 & 0 & 0 & 1 & 1 & 4 & 1 & 1 & 0 & 1 \\
\hline 308424 & 0 & 2 & 3 & 2 & 4 & 2 & 1 & 1 & 2 & 4 & 0 & 0 & 1 & 1 & 4 & 1 & 1 & 0 & 1 \\
\hline 308425 & 0 & 3 & 3 & 2 & 4 & 2 & 3 & 2 & 2 & 4 & 0 & 0 & 1 & 1 & 1 & 1 & 0 & 0 & 0 \\
\hline 308426 & 0 & 2 & 3 & 2 & 4 & 2 & 3 & 2 & 2 & 4 & 0 & 0 & 1 & 1 & 1 & 1 & 0 & 0 & 0 \\
\hline 308427 & 1 & 2 & 3 & 2 & 4 & 2 & 1 & 2 & 4 & 4 & 0 & 0 & 0 & 99 & 99 & 99 & 99 & 99 & 99 \\
\hline 308428 & 1 & 4 & 3 & 2 & 4 & 2 & 2 & 2 & 4 & 4 & 0 & 0 & 1 & 1 & 2 & 1 & 1 & 0 & 1 \\
\hline 308429 & 0 & 1 & 3 & 4 & 4 & 2 & 2 & 2 & 4 & 4 & 0 & 0 & 1 & 1 & 3 & 1 & 1 & 0 & 1 \\
\hline 3084210 & 0 & 2 & 3 & 2 & 4 & 2 & 2 & 1 & 2 & 4 & 0 & 0 & 0 & 99 & 99 & 99 & 99 & 99 & 99 \\
\hline 3084211 & 1 & 3 & 3 & 2 & 4 & 2 & 1 & 2 & 2 & 3 & 0 & 0 & 0 & 99 & 99 & 99 & 99 & 99 & 99 \\
\hline 3084212 & 0 & 3 & 3 & 1 & 4 & 2 & 2 & 1 & 2 & 1 & 0 & 0 & 0 & 99 & 99 & 99 & 99 & 99 & 99 \\
\hline 3084213 & 0 & 2 & 3 & 2 & 4 & 2 & 2 & 2 & 4 & 4 & 0 & 0 & 0 & 99 & 99 & 99 & 99 & 99 & 99 \\
\hline 3084214 & 0 & 2 & 3 & 2 & 4 & 2 & 2 & 1 & 4 & 4 & 0 & 0 & 0 & 99 & 99 & 99 & 99 & 99 & 99 \\
\hline 3084215 & 1 & 2 & 3 & 2 & 4 & 2 & 1 & 1 & 2 & 4 & 0 & 0 & 0 & 99 & 99 & 99 & 99 & 99 & 99 \\
\hline 3084216 & 1 & 3 & 3 & 2 & 4 & 2 & 2 & 2 & 2 & 4 & 0 & 0 & 0 & 99 & 99 & 99 & 99 & 99 & 99 \\
\hline 3084217 & 1 & 2 & 3 & 2 & 4 & 2 & 2 & 1 & 2 & 1 & 0 & 0 & 0 & 99 & 99 & 99 & 99 & 99 & 99 \\
\hline 3084218 & 1 & 2 & 3 & 2 & 4 & 2 & 2 & 1 & 4 & 1 & 0 & 0 & 1 & 1 & 3 & 1 & 1 & 0 & 1 \\
\hline 3084219 & 1 & 3 & 3 & 3 & 4 & 2 & 2 & 1 & 4 & 1 & 0 & 0 & 1 & 1 & 1 & 1 & 0 & 0 & 0 \\
\hline 3084220 & 1 & 4 & 3 & 4 & 4 & 5 & 3 & 2 & 4 & 1 & 1 & 1 & 0 & 99 & 99 & 99 & 99 & 99 & 99 \\
\hline 3084221 & 0 & 2 & 3 & 2 & 4 & 3 & 2 & 2 & 4 & 1 & 1 & 1 & 1 & 1 & 1 & 1 & 0 & 0 & 0 \\
\hline 3084222 & 0 & 4 & 3 & 3 & 4 & 1 & 2 & 2 & 4 & 1 & 0 & 0 & 0 & 99 & 99 & 99 & 99 & 99 & 99 \\
\hline 3084223 & 0 & 4 & 3 & 2 & 4 & 1 & 1 & 1 & 4 & 1 & 0 & 0 & 1 & 1 & 1 & 1 & 0 & 0 & 0 \\
\hline 3084224 & 0 & 2 & 3 & 3 & 4 & 1 & 2 & 2 & 4 & 1 & 0 & 0 & 0 & 99 & 99 & 99 & 99 & 99 & 99 \\
\hline 3084225 & 1 & 3 & 3 & 2 & 4 & 2 & 2 & 2 & 4 & 2 & 0 & 0 & 0 & 99 & 99 & 99 & 99 & 99 & 99 \\
\hline 3084226 & 0 & 2 & 3 & 4 & 4 & 1 & 3 & 2 & 4 & 3 & 0 & 0 & 1 & 1 & 1 & 1 & 0 & 0 & 0 \\
\hline 3084227 & 1 & 4 & 3 & 2 & 4 & 2 & 2 & 2 & 4 & 3 & 0 & 0 & 0 & 99 & 99 & 99 & 99 & 99 & 99 \\
\hline 3084228 & 1 & 5 & 3 & 1 & 4 & 2 & 2 & 1 & 4 & 2 & 0 & 0 & 0 & 99 & 99 & 99 & 99 & 99 & 99 \\
\hline 3084229 & 0 & 1 & 3 & 3 & 4 & 1 & 3 & 2 & 4 & 2 & 0 & 0 & 1 & 1 & 1 & 1 & 0 & 0 & 0 \\
\hline 3084230 & 1 & 4 & 3 & 2 & 4 & 5 & 2 & 2 & 4 & 1 & 1 & 1 & 0 & 99 & 99 & 99 & 99 & 99 & 99 \\
\hline 308431 & 0 & 3 & 3 & 2 & 4 & 1 & 2 & 2 & 2 & 4 & 0 & 0 & 1 & 1 & 1 & 1 & 0 & 0 & 0 \\
\hline 308432 & 1 & 5 & 3 & 4 & 4 & 5 & 2 & 1 & 2 & 4 & 0 & 0 & 1 & 1 & 2 & 1 & 1 & 0 & \\
\hline
\end{tabular}




\begin{tabular}{|c|c|c|c|c|c|c|c|c|c|c|c|c|c|c|c|c|c|c|c|}
\hline 308433 & 0 & 2 & 3 & 2 & 4 & 2 & 1 & 1 & 2 & 4 & 0 & 0 & 1 & 1 & 2 & 1 & 1 & 0 & 1 \\
\hline 308434 & 0 & 4 & 3 & 2 & 4 & 2 & 2 & 1 & 2 & 4 & 0 & 0 & 0 & 99 & 99 & 99 & 99 & 99 & 99 \\
\hline 308435 & 0 & 2 & 3 & 4 & 4 & 2 & 1 & 1 & 2 & 4 & 0 & 0 & 1 & 1 & 1 & 1 & 0 & 0 & 0 \\
\hline 308436 & 1 & 1 & 3 & 4 & 4 & 5 & 2 & 1 & 2 & 4 & 0 & 0 & 1 & 1 & 2 & 1 & 1 & 0 & 1 \\
\hline 308437 & 0 & 1 & 3 & 5 & 4 & 5 & 2 & 2 & 2 & 4 & 1 & 0 & 0 & 99 & 99 & 99 & 99 & 99 & 99 \\
\hline 308438 & 1 & 2 & 3 & 2 & 4 & 3 & 2 & 1 & 2 & 3 & 0 & 0 & 0 & 99 & 99 & 99 & 99 & 99 & 99 \\
\hline 308439 & 0 & 2 & 3 & 2 & 4 & 2 & 2 & 2 & 2 & 4 & 0 & 0 & 0 & 99 & 99 & 99 & 99 & 99 & 99 \\
\hline 3084310 & 1 & 3 & 3 & 3 & 4 & 2 & 1 & 2 & 2 & 3 & 0 & 0 & 1 & 1 & 1 & 1 & 0 & 0 & 0 \\
\hline 3084311 & 0 & 2 & 3 & 2 & 4 & 2 & 2 & 2 & 2 & 4 & 0 & 0 & 1 & 1 & 3 & 1 & 1 & 0 & 1 \\
\hline 3084312 & 0 & 2 & 3 & 2 & 2 & 1 & 2 & 2 & 4 & 1 & 0 & 0 & 0 & 99 & 99 & 99 & 99 & 99 & 99 \\
\hline 3084313 & 1 & 5 & 3 & 2 & 4 & 2 & 2 & 1 & 4 & 2 & 0 & 0 & 0 & 99 & 99 & 99 & 99 & 99 & 99 \\
\hline 3084314 & 0 & 1 & 3 & 4 & 4 & 1 & 2 & 2 & 4 & 4 & 0 & 0 & 0 & 99 & 99 & 99 & 99 & 99 & 99 \\
\hline 3084315 & 1 & 4 & 3 & 4 & 4 & 3 & 3 & 2 & 4 & 2 & 0 & 0 & 0 & 99 & 99 & 99 & 99 & 99 & 99 \\
\hline 3084316 & 1 & 1 & 3 & 2 & 4 & 2 & 3 & 1 & 4 & 4 & 0 & 0 & 1 & 1 & 1 & 6 & 0 & 1 & 1 \\
\hline 3084317 & 1 & 1 & 3 & 4 & 2 & 2 & 2 & 2 & 4 & 3 & 0 & 0 & 1 & 1 & 1 & 6 & 0 & 1 & 1 \\
\hline 3084318 & 0 & 1 & 3 & 2 & 4 & 1 & 2 & 1 & 4 & 4 & 0 & 0 & 0 & 99 & 99 & 99 & 99 & 99 & 99 \\
\hline 3084319 & 0 & 2 & 3 & 2 & 4 & 1 & 2 & 2 & 4 & 4 & 0 & 0 & 0 & 99 & 99 & 99 & 99 & 99 & 99 \\
\hline 3084320 & 1 & 2 & 3 & 3 & 4 & 2 & 3 & 2 & 4 & 4 & 0 & 0 & 0 & 99 & 99 & 99 & 99 & 99 & 99 \\
\hline 3084321 & 1 & 5 & 3 & 2 & 4 & 2 & 2 & 2 & 4 & 2 & 0 & 0 & 1 & 1 & 4 & 1 & 1 & 0 & 1 \\
\hline 3084322 & 0 & 3 & 3 & 2 & 4 & 3 & 3 & 2 & 4 & 2 & 0 & 0 & 0 & 99 & 99 & 99 & 99 & 99 & 99 \\
\hline 3084323 & 0 & 3 & 3 & 2 & 4 & 2 & 2 & 2 & 4 & 3 & 0 & 0 & 0 & 99 & 99 & 99 & 99 & 99 & 99 \\
\hline 3084324 & 1 & 1 & 3 & 2 & 4 & 2 & 3 & 1 & 4 & 4 & 0 & 0 & 0 & 99 & 99 & 99 & 99 & 99 & 99 \\
\hline 3084325 & 0 & 3 & 3 & 4 & 4 & 1 & 3 & 2 & 4 & 1 & 0 & 0 & 0 & 99 & 99 & 99 & 99 & 99 & 99 \\
\hline 3084326 & 0 & 1 & 3 & 2 & 4 & 2 & 1 & 1 & 4 & 4 & 0 & 0 & 0 & 99 & 99 & 99 & 99 & 99 & 99 \\
\hline 3084327 & 0 & 1 & 3 & 2 & 4 & 2 & 1 & 1 & 4 & 4 & 0 & 0 & 0 & 99 & 99 & 99 & 99 & 99 & 99 \\
\hline 3084328 & 0 & 1 & 3 & 5 & 4 & 5 & 2 & 2 & 4 & 4 & 0 & 0 & 0 & 99 & 99 & 99 & 99 & 99 & 99 \\
\hline 3084329 & 1 & 5 & 3 & 5 & 4 & 5 & 3 & 2 & 4 & 1 & 1 & 1 & 1 & 1 & 2 & 1 & 1 & 0 & 1 \\
\hline 3084330 & 0 & 2 & 3 & 2 & 4 & 2 & 2 & 1 & 4 & 4 & 0 & 0 & 0 & 99 & 99 & 99 & 99 & 99 & 99 \\
\hline 3084331 & 1 & 4 & 3 & 4 & 4 & 2 & 2 & 1 & 4 & 4 & 0 & 0 & 1 & 1 & 4 & 1 & 1 & 0 & 1 \\
\hline 3084332 & 1 & 2 & 3 & 5 & 4 & 5 & 2 & 1 & 4 & 3 & 1 & 1 & 1 & 1 & 1 & 6 & 0 & 1 & 1 \\
\hline 3084333 & 0 & 2 & 3 & 5 & 4 & 5 & 3 & 1 & 4 & 4 & 1 & 1 & 0 & 99 & 99 & 99 & 99 & 99 & 99 \\
\hline 3084334 & 1 & 4 & 3 & 5 & 4 & 5 & 3 & 2 & 4 & 4 & 1 & 1 & 0 & 99 & 99 & 99 & 99 & 99 & 99 \\
\hline 3084335 & 1 & 4 & 3 & 5 & 4 & 5 & 3 & 1 & 4 & 3 & 0 & 0 & 1 & 1 & 1 & 1 & 0 & 0 & 0 \\
\hline 3084336 & 1 & 4 & 3 & 5 & 3 & 5 & 3 & 2 & 4 & 4 & 1 & 1 & 1 & 1 & 1 & 1 & 0 & 0 & 0 \\
\hline 3084337 & 0 & 4 & 3 & 2 & 4 & 1 & 3 & 1 & 4 & 4 & 0 & 0 & 1 & 1 & 1 & 1 & 0 & 0 & 0 \\
\hline 3084338 & 1 & 3 & 3 & 4 & 4 & 3 & 3 & 2 & 4 & 3 & 1 & 0 & 0 & 99 & 99 & 99 & 99 & 99 & 99 \\
\hline 3084339 & 1 & 4 & 3 & 3 & 4 & 2 & 3 & 1 & 4 & 4 & 0 & 0 & 1 & 1 & 3 & 1 & 1 & 0 & 1 \\
\hline 308441 & 0 & 2 & 3 & 3 & 4 & 2 & 2 & 1 & 2 & 4 & 0 & 0 & 1 & 1 & 2 & 1 & 1 & 0 & 1 \\
\hline 308442 & 0 & 2 & 3 & 2 & 4 & 2 & 2 & 2 & 2 & 4 & 0 & 0 & 0 & 99 & 99 & 99 & 99 & 99 & 99 \\
\hline 308443 & 0 & 2 & 3 & 2 & 4 & 2 & 2 & 2 & 2 & 1 & 0 & 0 & 0 & 99 & 99 & 99 & 99 & 99 & 99 \\
\hline 308444 & 0 & 5 & 3 & 2 & 4 & 2 & 2 & 1 & 2 & 1 & 0 & 0 & 1 & 1 & 1 & 1 & 0 & 0 & 0 \\
\hline 308445 & 0 & 5 & 3 & 2 & 4 & 2 & 2 & 1 & 2 & 1 & 0 & 0 & 1 & 1 & 3 & 1 & 1 & 0 & 1 \\
\hline 308446 & 1 & 4 & 3 & 2 & 4 & 2 & 2 & 2 & 2 & 1 & 0 & 0 & 1 & 1 & 3 & 1 & 1 & 0 & 1 \\
\hline 308447 & 1 & 5 & 3 & 2 & 4 & 2 & 2 & 1 & 2 & 3 & 1 & 1 & 0 & 99 & 99 & 99 & 99 & 99 & 99 \\
\hline 308448 & 1 & 5 & 3 & 2 & 4 & 2 & 2 & 2 & 2 & 4 & 0 & 0 & 0 & 99 & 99 & 99 & 99 & 99 & 99 \\
\hline 308449 & 0 & 1 & 3 & 2 & 4 & 2 & 1 & 1 & 2 & 4 & 0 & 0 & 1 & 1 & 3 & 1 & 1 & 0 & 1 \\
\hline 3084410 & 1 & 3 & 3 & 3 & 4 & 2 & 2 & 1 & 2 & 4 & 0 & 0 & 0 & 99 & 99 & 99 & 99 & 99 & 99 \\
\hline 3084411 & 1 & 5 & 3 & 1 & 4 & 2 & 2 & 2 & 2 & 2 & 0 & 0 & 0 & 99 & 99 & 99 & 99 & 99 & 99 \\
\hline 3084412 & 1 & 3 & 3 & 4 & 4 & 3 & 3 & 1 & 2 & 1 & 1 & 0 & 1 & 1 & 4 & 6 & 1 & 1 & 1 \\
\hline 3084413 & 1 & 5 & 3 & 2 & 4 & 2 & 2 & 1 & 2 & 1 & 0 & 0 & 1 & 1 & 4 & 1 & 1 & 0 & 1 \\
\hline 3084414 & 1 & 3 & 3 & 4 & 4 & 5 & 3 & 2 & 2 & 1 & 0 & 0 & 1 & 1 & 1 & 1 & 0 & 0 & 0 \\
\hline 3084415 & 1 & 1 & 3 & 2 & 4 & 4 & 3 & 2 & 2 & 1 & 0 & 0 & 1 & 1 & 3 & 1 & 1 & 0 & 1 \\
\hline 3084416 & 0 & 3 & 3 & 2 & 4 & 2 & 2 & 1 & 2 & 1 & 0 & 0 & 0 & 99 & 99 & 99 & 99 & 99 & 99 \\
\hline 3084417 & 0 & 1 & 3 & 5 & 4 & 5 & 3 & 2 & 2 & 3 & 0 & 0 & 1 & 1 & 3 & 1 & 1 & 0 & 1 \\
\hline 3084418 & 1 & 2 & 3 & 3 & 4 & 2 & 3 & 1 & 2 & 3 & 1 & 1 & 0 & 99 & 99 & 99 & 99 & 99 & 99 \\
\hline 3084419 & 1 & 1 & 3 & 4 & 4 & 2 & 2 & 1 & 2 & 3 & 0 & 0 & 1 & 1 & 3 & 1 & 1 & 0 & \\
\hline
\end{tabular}




\begin{tabular}{|c|c|c|c|c|c|c|c|c|c|c|c|c|c|c|c|c|c|c|c|}
\hline 3084420 & 1 & 5 & 3 & 2 & 4 & 2 & 1 & 2 & 2 & 1 & 0 & 0 & 0 & 99 & 99 & 99 & \begin{tabular}{l|l}
99 \\
\end{tabular} & 99 & 99 \\
\hline 3084421 & 0 & 2 & 3 & 3 & 4 & 1 & 3 & 1 & 2 & 4 & 0 & 0 & 0 & 99 & 99 & 99 & 99 & 99 & 99 \\
\hline 3084422 & 0 & 2 & 3 & 2 & 4 & 2 & 2 & 2 & 2 & 4 & 0 & 0 & 0 & 99 & 99 & 99 & 99 & 99 & 99 \\
\hline 3084423 & 1 & 2 & 3 & 5 & 4 & 5 & 3 & 1 & 2 & 4 & 0 & 0 & 1 & 1 & 1 & 1 & 0 & 0 & 0 \\
\hline 3084424 & 1 & 1 & 3 & 5 & 4 & 5 & 3 & 1 & 2 & 4 & 0 & 0 & 1 & 1 & 1 & 6 & 0 & 1 & 1 \\
\hline 3084425 & 1 & 2 & 3 & 4 & 4 & 5 & 3 & 2 & 2 & 4 & 1 & 0 & 0 & 99 & 99 & 99 & 99 & 99 & $\overline{99}$ \\
\hline 3084426 & 0 & 1 & 3 & 5 & 4 & 5 & 3 & 1 & 2 & 3 & 1 & 0 & 1 & 1 & 1 & 1 & 0 & 0 & 0 \\
\hline 308451 & 0 & 4 & 3 & 1 & 4 & 2 & 2 & 2 & 3 & 2 & 0 & 0 & 1 & 1 & 1 & 1 & 0 & 0 & 0 \\
\hline 308452 & 1 & 2 & 3 & 2 & 4 & 2 & 1 & 2 & 3 & 2 & 0 & 0 & 1 & 1 & 1 & 4 & 0 & 1 & 1 \\
\hline 308453 & 0 & 3 & 3 & 2 & 4 & 2 & 2 & 1 & 3 & 2 & 0 & 0 & 0 & 99 & 99 & 99 & 99 & 99 & 99 \\
\hline 308454 & 0 & 2 & 3 & 2 & 4 & 2 & 1 & 2 & 3 & 3 & 0 & 0 & 0 & 99 & 99 & 99 & 99 & 99 & 99 \\
\hline 308455 & 0 & 4 & 3 & 1 & 4 & 2 & 1 & 1 & 3 & 2 & 0 & 0 & 1 & 1 & 1 & 6 & 0 & 1 & 1 \\
\hline 308456 & 1 & 2 & 3 & 2 & 4 & 2 & 2 & 2 & 1 & 2 & 0 & 0 & 1 & 1 & 1 & 1 & 0 & 0 & 0 \\
\hline 308457 & 1 & 2 & 3 & 2 & 4 & 2 & 1 & 2 & 3 & 3 & 0 & 0 & 1 & 1 & 1 & 4 & 0 & 1 & 1 \\
\hline 308458 & 0 & 3 & 3 & 2 & 4 & 2 & 2 & 1 & 3 & 3 & 0 & 0 & 0 & 99 & 99 & 99 & 99 & 99 & 99 \\
\hline 308459 & 0 & 2 & 3 & 1 & 4 & 2 & 1 & 1 & 3 & 2 & 0 & 0 & 1 & 1 & 1 & 4 & 0 & 1 & 1 \\
\hline 3084510 & 0 & 2 & 3 & 2 & 4 & 2 & 2 & 1 & 3 & 2 & 0 & 0 & 1 & 1 & 1 & 4 & 0 & 1 & 1 \\
\hline 3084511 & 1 & 4 & 3 & 1 & 4 & 2 & 1 & 2 & 3 & 2 & 0 & 0 & 1 & 1 & 1 & 4 & 0 & 1 & 1 \\
\hline 3084512 & 0 & 2 & 3 & 2 & 4 & 2 & 1 & 2 & 3 & 2 & 0 & 0 & 1 & 1 & 1 & 4 & 0 & 1 & 1 \\
\hline 3084513 & 0 & 1 & 3 & 2 & 4 & 2 & 1 & 1 & 3 & 2 & 0 & 0 & 1 & 1 & 1 & 4 & 0 & 1 & 1 \\
\hline 3084514 & 0 & 1 & 3 & 2 & 4 & 2 & 1 & 1 & 3 & 2 & 0 & 0 & 1 & 1 & 1 & 4 & 0 & 1 & 1 \\
\hline 3084515 & 1 & 4 & 3 & 1 & 4 & 2 & 1 & 1 & 3 & 2 & 0 & 0 & 1 & 1 & 1 & 4 & 0 & 1 & 1 \\
\hline 3084516 & 1 & 4 & 3 & 2 & 4 & 2 & 1 & 2 & 3 & 2 & 0 & 0 & 1 & 1 & 1 & 4 & 0 & 1 & 1 \\
\hline 3084517 & 0 & 4 & 3 & 2 & 4 & 2 & 1 & 2 & 3 & 2 & 0 & 0 & 1 & 1 & 3 & 4 & 1 & 1 & 1 \\
\hline 3084518 & 1 & 3 & 3 & 2 & 4 & 2 & 1 & 2 & 3 & 2 & 0 & 0 & 1 & 1 & 1 & 4 & 0 & 1 & 1 \\
\hline 3084519 & 0 & 4 & 3 & 1 & 4 & 2 & 1 & 1 & 3 & 2 & 0 & 0 & 1 & 1 & 1 & 4 & 0 & 1 & 1 \\
\hline 3084520 & 0 & 5 & 3 & 2 & 4 & 2 & 1 & 1 & 3 & 2 & 0 & 0 & 1 & 1 & 1 & 4 & 0 & 1 & 1 \\
\hline 3084521 & 0 & 4 & 3 & 2 & 4 & 2 & 1 & 2 & 3 & 2 & 0 & 0 & 1 & 1 & 1 & 4 & 0 & 1 & 1 \\
\hline 3084522 & 0 & 2 & 3 & 2 & 4 & 2 & 2 & 2 & 3 & 2 & 0 & 0 & 1 & 1 & 1 & 6 & 0 & 1 & 1 \\
\hline 3084523 & 0 & 1 & 3 & 2 & 4 & 2 & 1 & 1 & 3 & 2 & 0 & 0 & 1 & 1 & 1 & 6 & 0 & 1 & 1 \\
\hline 3084524 & 1 & 4 & 3 & 2 & 4 & 2 & 1 & 2 & 3 & 2 & 0 & 0 & 1 & 1 & 1 & 1 & 0 & 0 & 0 \\
\hline 3084525 & 1 & 5 & 3 & 2 & 4 & 2 & 1 & 1 & 3 & 2 & 0 & 0 & 1 & 1 & 1 & 4 & 0 & 1 & 1 \\
\hline 308461 & 0 & 4 & 3 & 3 & 4 & 2 & 3 & 2 & 3 & 2 & 1 & 1 & 1 & 1 & 1 & 6 & 0 & 1 & 1 \\
\hline 308462 & 1 & 3 & 3 & 1 & 4 & 2 & 1 & 1 & 3 & 2 & 0 & 0 & 1 & 1 & 1 & 1 & 0 & 0 & 0 \\
\hline 308463 & 1 & 2 & 3 & 2 & 4 & 2 & 2 & 1 & 3 & 2 & 0 & 0 & 1 & 1 & 1 & 4 & 0 & 1 & 1 \\
\hline 308464 & 1 & 4 & 3 & 1 & 4 & 2 & 1 & 2 & 3 & 2 & 0 & 0 & 1 & 1 & 1 & 4 & 0 & 1 & 1 \\
\hline 308465 & 0 & 5 & 3 & 2 & 4 & 2 & 1 & 1 & 3 & 2 & 0 & 0 & 1 & 1 & 1 & 4 & 0 & 1 & 1 \\
\hline 308466 & 1 & 3 & 3 & 2 & 4 & 2 & 1 & 1 & 3 & 2 & 0 & 0 & 1 & 1 & 2 & 1 & 1 & 0 & 1 \\
\hline 308467 & 0 & 4 & 3 & 2 & 4 & 2 & 2 & 1 & 3 & 2 & 0 & 0 & 1 & 1 & 1 & 6 & 0 & 1 & 1 \\
\hline 308468 & 0 & 3 & 3 & 2 & 4 & 2 & 2 & 2 & 3 & 2 & 0 & 0 & 1 & 1 & 1 & 1 & 0 & 0 & 0 \\
\hline 308469 & 0 & 5 & 3 & 2 & 4 & 2 & 1 & 1 & 3 & 2 & 0 & 0 & 1 & 1 & 1 & 4 & 0 & 1 & 1 \\
\hline 3084610 & 1 & 2 & 3 & 2 & 4 & 2 & 1 & 1 & 3 & 2 & 0 & 0 & 1 & 1 & 1 & 1 & 0 & 0 & 0 \\
\hline 3084611 & 1 & 2 & 3 & 2 & 4 & 2 & 1 & 2 & 3 & 2 & 0 & 0 & 1 & 1 & 1 & 4 & 0 & 1 & 1 \\
\hline 3084612 & 0 & 3 & 3 & 2 & 4 & 2 & 2 & 1 & 3 & 2 & 0 & 0 & 1 & 1 & 1 & 6 & 0 & 1 & 1 \\
\hline 3084613 & 0 & 3 & 3 & 4 & 4 & 2 & 2 & 1 & 3 & 2 & 0 & 0 & 1 & 1 & 1 & 1 & 0 & 0 & 0 \\
\hline 3084614 & 1 & 5 & 3 & 2 & 4 & 2 & 1 & 1 & 3 & 2 & 0 & 0 & 1 & 1 & 1 & 4 & 0 & 1 & 1 \\
\hline 3084615 & 1 & 5 & 3 & 2 & 4 & 2 & 2 & 1 & 3 & 2 & 0 & 0 & 1 & 1 & 1 & 4 & 0 & 1 & 1 \\
\hline 3084616 & 1 & 4 & 3 & 4 & 4 & 2 & 1 & 1 & 3 & 2 & 0 & 0 & 1 & 1 & 1 & 4 & 0 & 1 & 1 \\
\hline 3084617 & 0 & 4 & 3 & 2 & 4 & 2 & 1 & 2 & 3 & 2 & 0 & 0 & 1 & 1 & 1 & 4 & 0 & 1 & 1 \\
\hline 3084618 & 1 & 4 & 3 & 2 & 4 & 2 & 1 & 2 & 3 & 2 & 0 & 0 & 1 & 1 & 1 & 4 & 0 & 1 & 1 \\
\hline 3084619 & 1 & 4 & 3 & 2 & 4 & 2 & 1 & 2 & 3 & 2 & 0 & 0 & 1 & 1 & 1 & 4 & 0 & 1 & 1 \\
\hline 3084620 & 1 & 3 & 3 & 2 & 4 & 2 & 1 & 1 & 3 & 2 & 0 & 0 & 1 & 1 & 1 & 4 & 0 & 1 & 1 \\
\hline 3084621 & 0 & 5 & 3 & 1 & 4 & 2 & 1 & 2 & 3 & 2 & 0 & 0 & 1 & 1 & 1 & 4 & 0 & 1 & 1 \\
\hline 3084622 & 1 & 3 & 3 & 2 & 4 & 2 & 1 & 1 & 3 & 2 & 0 & 0 & 1 & 1 & 1 & 4 & 0 & 1 & 1 \\
\hline 3084623 & 1 & 4 & 3 & 2 & 4 & 2 & 1 & 1 & 3 & 2 & 0 & 0 & 1 & 1 & 1 & 4 & 0 & 1 & 1 \\
\hline 3084624 & 1 & 3 & 3 & 2 & 4 & 2 & 1 & 2 & 3 & 2 & 0 & 0 & 1 & 1 & 1 & 4 & 0 & 1 & \\
\hline
\end{tabular}




\begin{tabular}{|c|c|c|c|c|c|c|c|c|c|c|c|c|c|c|c|c|c|c|c|}
\hline 3084625 & 0 & 4 & 3 & 2 & 4 & 2 & 1 & 1 & 3 & 2 & 0 & 0 & 1 & 1 & 1 & 4 & 0 & 1 & 1 \\
\hline 309471 & 1 & 4 & 3 & 4 & 4 & 2 & 2 & 2 & 4 & 2 & 1 & 1 & 1 & 1 & 1 & 1 & 0 & 0 & 0 \\
\hline 309472 & 0 & 3 & 3 & 3 & 4 & 2 & 1 & 1 & 4 & 1 & 0 & 0 & 1 & 1 & 2 & 1 & 1 & 0 & 1 \\
\hline 309473 & 1 & 3 & 3 & 2 & 4 & 2 & 1 & 2 & 4 & 2 & 0 & 0 & 1 & 1 & 1 & 1 & 0 & 0 & 0 \\
\hline 309474 & 1 & 4 & 3 & 2 & 4 & 2 & 1 & 2 & 4 & 1 & 1 & 0 & 1 & 1 & 1 & 1 & 0 & 0 & 0 \\
\hline 309475 & 0 & 2 & 3 & 2 & 4 & 2 & 1 & 1 & 4 & 1 & 1 & 1 & 1 & 1 & 1 & 1 & 0 & 0 & 0 \\
\hline 309476 & 0 & 3 & 3 & 4 & 4 & 1 & 3 & 2 & 4 & 4 & 1 & 1 & 1 & 1 & 1 & 1 & 0 & 0 & 0 \\
\hline 309477 & 1 & 2 & 3 & 2 & 4 & 2 & 2 & 2 & 4 & 1 & 0 & 0 & 1 & 1 & 2 & 1 & 1 & 0 & 1 \\
\hline 309478 & 1 & 4 & 3 & 4 & 4 & 2 & 1 & 2 & 4 & 1 & 1 & 0 & 1 & 1 & 1 & 1 & 0 & 0 & 0 \\
\hline 309479 & 1 & 5 & 3 & 2 & 4 & 2 & 1 & 1 & 4 & 4 & 1 & 0 & 1 & 1 & 2 & 4 & 1 & 1 & 1 \\
\hline 3094710 & 1 & 2 & 3 & 2 & 4 & 2 & 3 & 1 & 4 & 4 & 0 & 0 & 1 & 1 & 2 & 1 & 1 & 0 & 1 \\
\hline 3094711 & 0 & 2 & 3 & 2 & 4 & 2 & 1 & 1 & 4 & 4 & 0 & 0 & 1 & 1 & 2 & 1 & 1 & 0 & 1 \\
\hline 3094712 & 0 & 2 & 3 & 5 & 4 & 5 & 2 & 2 & 4 & 3 & 0 & 0 & 1 & 1 & 1 & 1 & 0 & 0 & 0 \\
\hline 3094713 & 1 & 1 & 3 & 2 & 4 & 2 & 2 & 2 & 4 & 4 & 0 & 0 & 1 & 1 & 2 & 4 & 1 & 1 & 1 \\
\hline 3094714 & 1 & 1 & 3 & 2 & 4 & 2 & 1 & 1 & 4 & 4 & 0 & 0 & 1 & 1 & 3 & 1 & 1 & 0 & 1 \\
\hline 3094715 & 1 & 4 & 3 & 2 & 4 & 2 & 1 & 1 & 4 & 4 & 0 & 0 & 1 & 1 & 1 & 4 & 0 & 1 & 1 \\
\hline 3094716 & 1 & 2 & 3 & 2 & 4 & 2 & 2 & 2 & 4 & 4 & 0 & 0 & 1 & 1 & 3 & 1 & 1 & 0 & 1 \\
\hline 3094717 & 1 & 2 & 3 & 5 & 4 & 5 & 3 & 1 & 4 & 1 & 1 & 0 & 1 & 1 & 1 & 1 & 0 & 0 & 0 \\
\hline 3094718 & 1 & 2 & 3 & 2 & 4 & 2 & 1 & 2 & 4 & 2 & 0 & 0 & 1 & 1 & 3 & 1 & 1 & 0 & 1 \\
\hline 3094719 & 0 & 2 & 3 & 3 & 4 & 2 & 2 & 2 & 4 & 2 & 0 & 0 & 1 & 1 & 2 & 1 & 1 & 0 & 1 \\
\hline 3094720 & 0 & 4 & 3 & 3 & 4 & 2 & 1 & 1 & 4 & 2 & 0 & 0 & 1 & 1 & 2 & 1 & 1 & 0 & 1 \\
\hline 3094721 & 1 & 3 & 3 & 2 & 4 & 2 & 1 & 2 & 4 & 2 & 0 & 0 & 1 & 1 & 2 & 1 & 1 & 0 & 1 \\
\hline 3094722 & 0 & 3 & 3 & 2 & 4 & 2 & 1 & 2 & 4 & 2 & 0 & 0 & 1 & 1 & 2 & 1 & 1 & 0 & 1 \\
\hline 3094723 & 1 & 3 & 3 & 3 & 4 & 2 & 1 & 2 & 4 & 2 & 0 & 0 & 1 & 1 & 2 & 1 & 1 & 0 & 1 \\
\hline 3094724 & 0 & 1 & 3 & 5 & 4 & 5 & 2 & 2 & 4 & 1 & 0 & 0 & 1 & 1 & 2 & 1 & 1 & 0 & 1 \\
\hline 3094725 & 1 & 5 & 3 & 2 & 4 & 2 & 1 & 1 & 4 & 1 & 0 & 0 & 1 & 1 & 2 & 1 & 1 & 0 & 1 \\
\hline 3094726 & 0 & 4 & 3 & 2 & 4 & 2 & 1 & 1 & 4 & 1 & 0 & 0 & 1 & 1 & 2 & 1 & 1 & 0 & 1 \\
\hline 3094727 & 1 & 3 & 3 & 3 & 4 & 2 & 1 & 2 & 4 & 1 & 0 & 0 & 1 & 1 & 2 & 1 & 1 & 0 & 1 \\
\hline 3094728 & 0 & 1 & 3 & 4 & 4 & 2 & 2 & 1 & 4 & 1 & 0 & 0 & 1 & 1 & 2 & 1 & 1 & 0 & 1 \\
\hline 3094729 & 0 & 3 & 3 & 4 & 4 & 2 & 1 & 1 & 4 & 2 & 0 & 0 & 1 & 1 & 2 & 1 & 1 & 0 & 1 \\
\hline 3094730 & 1 & 3 & 3 & 3 & 4 & 2 & 1 & 2 & 4 & 2 & 0 & 0 & 1 & 1 & 2 & 1 & 1 & 0 & 1 \\
\hline 3094731 & 0 & 3 & 3 & 2 & 4 & 2 & 1 & 2 & 4 & 2 & 0 & 0 & 1 & 1 & 2 & 1 & 1 & 0 & 1 \\
\hline 3094732 & 1 & 2 & 3 & 2 & 4 & 2 & 1 & 1 & 4 & 2 & 0 & 0 & 1 & 1 & 2 & 1 & 1 & 0 & 1 \\
\hline 3094733 & 0 & 2 & 3 & 2 & 4 & 2 & 2 & 2 & 4 & 1 & 0 & 0 & 1 & 1 & 2 & 1 & 1 & 0 & 1 \\
\hline 3094734 & 0 & 2 & 3 & 4 & 4 & 2 & 3 & 2 & 4 & 1 & 0 & 0 & 1 & 1 & 2 & 1 & 1 & 0 & 1 \\
\hline 3094735 & 0 & 3 & 3 & 4 & 4 & 2 & 1 & 1 & 4 & 2 & 0 & 0 & 1 & 1 & 2 & 1 & 1 & 0 & 1 \\
\hline 3094736 & 0 & 3 & 3 & 4 & 4 & 2 & 1 & 1 & 4 & 1 & 0 & 0 & 1 & 1 & 2 & 1 & 1 & 0 & 1 \\
\hline 3094737 & 0 & 4 & 3 & 3 & 4 & 2 & 2 & 1 & 4 & 4 & 0 & 0 & 1 & 1 & 3 & 1 & 1 & 0 & 1 \\
\hline 3094738 & 0 & 3 & 3 & 3 & 4 & 2 & 1 & 1 & 4 & 2 & 0 & 0 & 1 & 1 & 2 & 1 & 1 & 0 & 1 \\
\hline 3094739 & 0 & 3 & 3 & 2 & 4 & 2 & 1 & 2 & 4 & 2 & 0 & 0 & 1 & 1 & 4 & 1 & 1 & 0 & 1 \\
\hline 3094740 & 0 & 3 & 3 & 4 & 4 & 2 & 2 & 1 & 4 & 2 & 1 & 0 & 1 & 1 & 1 & 1 & 0 & 0 & 0 \\
\hline 309481 & 1 & 2 & 3 & 3 & 4 & 2 & 1 & 1 & 2 & 1 & 0 & 0 & 1 & 1 & 2 & 1 & 1 & 0 & 1 \\
\hline 309482 & 1 & 2 & 3 & 3 & 4 & 2 & 1 & 1 & 2 & 4 & 0 & 0 & 1 & 1 & 2 & 1 & 1 & 0 & 1 \\
\hline 309483 & 0 & 2 & 3 & 5 & 4 & 5 & 1 & 2 & 2 & 1 & 0 & 0 & 1 & 1 & 3 & 1 & 1 & 0 & 1 \\
\hline 309484 & 0 & 5 & 3 & 2 & 4 & 2 & 1 & 2 & 2 & 1 & 0 & 0 & 1 & 1 & 3 & 1 & 1 & 0 & 1 \\
\hline 309485 & 0 & 2 & 3 & 4 & 4 & 2 & 2 & 2 & 2 & 1 & 0 & 0 & 1 & 1 & 2 & 1 & 1 & 0 & 1 \\
\hline 309486 & 0 & 3 & 3 & 2 & 4 & 2 & 2 & 2 & 2 & 1 & 0 & 0 & 1 & 1 & 1 & 1 & 0 & 0 & 0 \\
\hline 309487 & 0 & 3 & 3 & 2 & 4 & 2 & 1 & 2 & 2 & 1 & 0 & 0 & 1 & 1 & 3 & 1 & 1 & 0 & 1 \\
\hline 309488 & 0 & 4 & 3 & 2 & 4 & 2 & 1 & 2 & 2 & 1 & 0 & 0 & 1 & 1 & 1 & 1 & 0 & 0 & 0 \\
\hline 309489 & 0 & 3 & 3 & 2 & 4 & 2 & 2 & 1 & 2 & 1 & 0 & 0 & 1 & 1 & 2 & 1 & 1 & 0 & 1 \\
\hline 3094810 & 0 & 3 & 3 & 2 & 4 & 2 & 1 & 1 & 2 & 2 & 0 & 0 & 1 & 1 & 3 & 1 & 1 & 0 & 1 \\
\hline 3094811 & 0 & 4 & 3 & 2 & 4 & 2 & 1 & 2 & 2 & 2 & 0 & 0 & 1 & 1 & 3 & 1 & 1 & 0 & 1 \\
\hline 3094812 & 1 & 5 & 3 & 2 & 4 & 2 & 1 & 2 & 2 & 2 & 0 & 0 & 1 & 1 & 2 & 1 & 1 & 0 & 1 \\
\hline 3094813 & 1 & 3 & 3 & 2 & 4 & 2 & 1 & 1 & 2 & 1 & 0 & 0 & 1 & 1 & 1 & 1 & 0 & 0 & 0 \\
\hline 3094814 & 1 & 2 & 3 & 2 & 4 & 2 & 2 & 1 & 2 & 1 & 0 & 0 & 1 & 1 & 3 & 1 & 1 & 0 & 1 \\
\hline 3094815 & 1 & 2 & 3 & 4 & 4 & 2 & 1 & 2 & 2 & 1 & 0 & 0 & 1 & 1 & 2 & 1 & 1 & 0 & 1 \\
\hline
\end{tabular}




\begin{tabular}{|c|c|c|c|c|c|c|c|c|c|c|c|c|c|c|c|c|c|c|c|}
\hline 3094816 & 0 & 2 & 3 & 2 & 4 & 2 & 1 & 2 & 2 & 2 & 0 & 0 & 1 & 1 & 2 & 1 & 1 & 0 & 1 \\
\hline 3094817 & 1 & 2 & 3 & 2 & 4 & 2 & 1 & 1 & 2 & 2 & 0 & 0 & 1 & 1 & 3 & 1 & 1 & 0 & 1 \\
\hline 3094818 & 1 & 1 & 3 & 2 & 4 & 2 & 1 & 1 & 2 & 2 & 0 & 0 & 1 & 1 & 3 & 1 & 1 & 0 & 1 \\
\hline 3094819 & 1 & 3 & 3 & 2 & 4 & 2 & 1 & 2 & 2 & 2 & 0 & 0 & 1 & 1 & 3 & 1 & 1 & 0 & 1 \\
\hline 3094820 & 0 & 2 & 3 & 2 & 4 & 2 & 1 & 1 & 2 & 2 & 0 & 0 & 1 & 1 & 2 & 1 & 1 & 0 & 1 \\
\hline 3094821 & 1 & 3 & 3 & 4 & 4 & 2 & 1 & 2 & 2 & 2 & 0 & 0 & 1 & 1 & 3 & 1 & 1 & 0 & 1 \\
\hline 3094822 & 1 & 5 & 3 & 2 & 4 & 2 & 1 & 2 & 2 & 1 & 0 & 0 & 1 & 1 & 2 & 1 & 1 & 0 & 1 \\
\hline 3094823 & 0 & 4 & 3 & 2 & 4 & 2 & 1 & 1 & 2 & 1 & 0 & 0 & 1 & 1 & 2 & 1 & 1 & 0 & 1 \\
\hline 3094824 & 1 & 5 & 3 & 2 & 4 & 2 & 1 & 2 & 2 & 2 & 0 & 0 & 1 & 1 & 2 & 1 & 1 & 0 & 1 \\
\hline 3094825 & 1 & 4 & 3 & 5 & 4 & 2 & 2 & 2 & 2 & 2 & 0 & 0 & 1 & 1 & 2 & 1 & 1 & 0 & 1 \\
\hline 309491 & 1 & 4 & 3 & 2 & 4 & 2 & 1 & 2 & 2 & 1 & 0 & 0 & 1 & 1 & 1 & 4 & 0 & 1 & 1 \\
\hline 309492 & 0 & 2 & 3 & 2 & 4 & 2 & 1 & 1 & 2 & 4 & 0 & 0 & 1 & 1 & 4 & 1 & 1 & 0 & 1 \\
\hline 309493 & 0 & 4 & 3 & 2 & 4 & 2 & 1 & 2 & 2 & 4 & 0 & 0 & 1 & 1 & 2 & 1 & 1 & 0 & 1 \\
\hline 309494 & 0 & 5 & 3 & 2 & 4 & 2 & 1 & 1 & 2 & 3 & 0 & 0 & 1 & 1 & 3 & 1 & 1 & 0 & 1 \\
\hline 309495 & 0 & 2 & 3 & 2 & 4 & 2 & 1 & 1 & 2 & 1 & 0 & 0 & 1 & 1 & 2 & 1 & 1 & 0 & 1 \\
\hline 309496 & 1 & 3 & 3 & 5 & 4 & 5 & 2 & 1 & 2 & 1 & 1 & 1 & 1 & 1 & 1 & 1 & 0 & 0 & 0 \\
\hline 309497 & 1 & 4 & 3 & 2 & 4 & 2 & 1 & 1 & 2 & 2 & 0 & 0 & 1 & 1 & 2 & 1 & 1 & 0 & 1 \\
\hline 309498 & 0 & 1 & 3 & 2 & 4 & 2 & 1 & 1 & 2 & 4 & 0 & 0 & 1 & 1 & 3 & 1 & 1 & 0 & 1 \\
\hline 309499 & 0 & 3 & 3 & 2 & 4 & 2 & 1 & 2 & 2 & 4 & 0 & 0 & 1 & 1 & 3 & 1 & 1 & 0 & 1 \\
\hline 3094910 & 1 & 3 & 3 & 3 & 4 & 2 & 2 & 2 & 2 & 4 & 0 & 0 & 1 & 1 & 4 & 1 & 1 & 0 & 1 \\
\hline 3094911 & 0 & 3 & 3 & 2 & 4 & 2 & 1 & 2 & 2 & 4 & 0 & 0 & 1 & 1 & 3 & 1 & 1 & 0 & 1 \\
\hline 3094912 & 0 & 2 & 3 & 5 & 4 & 5 & 2 & 1 & 2 & 4 & 0 & 0 & 1 & 1 & 4 & 1 & 1 & 0 & 1 \\
\hline 3094913 & 1 & 2 & 3 & 2 & 4 & 2 & 1 & 1 & 3 & 4 & 0 & 0 & 1 & 1 & 2 & 1 & 1 & 0 & 1 \\
\hline 3094914 & 1 & 2 & 3 & 3 & 4 & 2 & 1 & 2 & 2 & 1 & 0 & 0 & 1 & 1 & 1 & 1 & 0 & 0 & 0 \\
\hline 3094915 & 1 & 3 & 3 & 2 & 4 & 2 & 1 & 1 & 2 & 1 & 0 & 0 & 1 & 1 & 2 & 1 & 1 & 0 & 1 \\
\hline 3094916 & 1 & 4 & 3 & 2 & 4 & 2 & 1 & 1 & 2 & 1 & 0 & 0 & 1 & 1 & 1 & 1 & 0 & 0 & 0 \\
\hline 3094917 & 1 & 2 & 3 & 2 & 4 & 2 & 1 & 1 & 2 & 1 & 0 & 0 & 1 & 1 & 1 & 1 & 0 & 0 & 0 \\
\hline 3094918 & 0 & 2 & 3 & 4 & 4 & 2 & 1 & 2 & 2 & 4 & 0 & 0 & 1 & 1 & 1 & 4 & 0 & 1 & 1 \\
\hline 3094919 & 0 & 2 & 3 & 2 & 4 & 2 & 1 & 2 & 2 & 4 & 0 & 0 & 1 & 1 & 3 & 1 & 1 & 0 & 1 \\
\hline 3094920 & 0 & 3 & 3 & 2 & 4 & 2 & 2 & 1 & 2 & 4 & 1 & 0 & 1 & 1 & 3 & 1 & 1 & 0 & 1 \\
\hline 3094921 & 1 & 3 & 3 & 2 & 4 & 2 & 1 & 2 & 2 & 4 & 0 & 0 & 1 & 1 & 1 & 4 & 0 & 1 & 1 \\
\hline 3094922 & 0 & 3 & 3 & 2 & 4 & 2 & 1 & 2 & 2 & 4 & 0 & 0 & 1 & 1 & 3 & 1 & 1 & 0 & 1 \\
\hline 3094923 & 0 & 2 & 3 & 2 & 4 & 2 & 1 & 1 & 2 & 1 & 0 & 0 & 1 & 1 & 1 & 1 & 0 & 0 & 0 \\
\hline 3094924 & 0 & 1 & 3 & 5 & 4 & 5 & 3 & 1 & 2 & 4 & 0 & 0 & 1 & 1 & 3 & 1 & 1 & 0 & 1 \\
\hline 3094925 & 0 & 2 & 3 & 5 & 4 & 5 & 1 & 2 & 2 & 4 & 0 & 0 & 1 & 1 & 3 & 1 & 1 & 0 & 1 \\
\hline 3094926 & 1 & 4 & 3 & 4 & 4 & 2 & 2 & 1 & 2 & 1 & 0 & 0 & 1 & 1 & 1 & 1 & 0 & 0 & 0 \\
\hline 3094927 & 1 & 4 & 3 & 2 & 4 & 2 & 2 & 1 & 2 & 1 & 0 & 0 & 1 & 1 & 1 & 4 & 0 & 1 & 1 \\
\hline 3094928 & 1 & 2 & 3 & 5 & 4 & 5 & 2 & 1 & 2 & 1 & 1 & 1 & 1 & 1 & 1 & 1 & 0 & 0 & 0 \\
\hline 3094929 & 1 & 1 & 3 & 2 & 4 & 2 & 1 & 1 & 2 & 1 & 0 & 0 & 1 & 1 & 2 & 1 & 1 & 0 & 1 \\
\hline 3094930 & 1 & 3 & 3 & 2 & 4 & 2 & 1 & 2 & 2 & 1 & 0 & 0 & 1 & 1 & 2 & 1 & 1 & 0 & 1 \\
\hline
\end{tabular}

\section{Variables explanation}

RID Respondent ID

G Gender of participants

0 Female

1 Male

AG Age group of participants

$$
\begin{array}{ll}
1 & <30 \\
2 & 30-39 \\
3 & 40-49 \\
4 & 50-59 \\
5 & \geq 60
\end{array}
$$


1 East Sumba

2 Belu

3 East Manggarai

Edu Education level of participants

1 No education

2 Primary school

3 Junior high school

4 Senior high school'

5 Diploma or above

Eth Ethnicity in ENTP

1 Sumba

2 Others

3 Timor

4 Manggarai

Job Occupation of participants

1 Housewife

2 Farmer

3 Enterpreneur/Salesman

4 Other

5 Govt. or non-govt. employment

SES Social economic status of participants

1 Low

2 Average

3 High

FZ Family size

$1 \leq 4$

$2>4$

$\mathrm{HF} \quad$ The nearest health facilities

1 Village maternity posts

2 Village health Post

3 Subsidiary Public Health centres

4 Public Health centres

DHF Distance to the nearest health facilities

$$
\begin{array}{ll}
1 & <1 \mathrm{Km} \\
2 & 1-2 \mathrm{Km} \\
3 & 2-3 \mathrm{Km} \\
4 & \geq 3 \mathrm{Km}
\end{array}
$$

Inc1 Category of Household Income based on Provincial Minimum Wages (PMW)

0 Less than PMW

1 At least PMW

Inc2 Category of Household Income based on Indonesia Poverty Line (IPL)

0 Less than IPL

1 At least IPL

Q1 Hearing malaria term

0 No

1 Yes

Q2 Seeking treatment if participants or their family members have symptoms of malaria

0 No

1 Yes

99 Not applicable 
1 One day (Within 24 hours)

22 days

33 days

44 days or more

5 I did not go for treatment

99 Not applicable

Q4 Place for seeking treatment if participants or their family members have symptoms of malaria

1 Public health facilities

2 Private health facilities

4 Traditional healer

5 Self-treatment

6 Buying medicine at kiosk

7 Self-treatment with consuming papaya leaves

99 Not applicable

O1 Seeking treatment after 24 hours

$0 \quad$ No

1 Yes

99 Not applicable

O2 Seeking treatment at non-health facilities

$0 \quad$ No

1 Yes

99 Not applicable

O3 Poor understanding of appropriate malaria treatment-seeking behaviour (AMTSB)

(Seeking treatment after 24 hours or at non-health facilities)

$0 \quad$ No

1 Yes

99 Not applicable 